SIMULATION OF LOCAL SITE RESPONSE TO EARTHQUAKES

S. M. Day, H. E. Read, H. J. Swanger, and $K$. J. Cheverton

Systems, Science and Software P.0. Box 1620

La Jolla, Ca. 92038

USGS CONTRACT NO. 14-08-0001-17776

Supported by the EARTHQUAKE HAZARDS REDUCTION PROGRAM

OPEN-FILE NO. $81-51$

U.S. Geological Survey

OPEN FILE REPORT

This report was prepared under contract to the U.S. Geological Survey and has not been reviewed for conformity with USGS editorial standards and stratigraphic nomenclature. Opinions and conclusions expressed herein do not necessarily represent those of the USGS. Any use of trade names is for descriptive purposes only and does not imply endorsement by the USGS. 


\author{
by \\ S. M. Day (Principal Investigator) \\ H. E. Read \\ H. J. Swanger \\ K. J. Cheverton \\ Systems, Science and Software \\ P.0. Box 1620 \\ La Jolla, California 92038 \\ Summary of Final Contract Report
}

\title{
Objective
}

The objective of this investigation is to develop and evaluate a new analytical approach for estimating site-dependent earthquake ground motion for engineering design purposes. The analytical approach is based on assuming physical models for the earthquake source, path wave-propagation effects, and nonlinear soil response at the site, then calculating ground motion using a combination of seismic (layer matrix) and finite element methods. The approach is structured to potentially provide a three-dimensional ground motion capability, although the nonlinear soil response calculations are here limited to one dimension. The predictive capabilities of this approach were examined by comparing ground motion calculations to empirically derived ground motion trends and to ground motion simulations performed using the equivalent linear method.

\section{Principal Results}

The most significant results of this work are the following: (1) A superior soil model, the endochronic model, has been introduced to site evaluation studies. (2) Comparison of the endochronic model with the conventional equivalent linear model has shown the latter to yield excessively conservative ground motion predictions for loose soil deposits in the near field.

\section{Summary}

For distances less than about $20 \mathrm{kilometers,} \mathrm{there} \mathrm{is} \mathrm{minimal}$ earthquake ground motion data available, and substantial disagreement exists among empirical relations for predicting design motion characteristics in this distance range. This disagreement reflects the uncertainties associated with extrapolation of the empirical relations to short distances, where data is sparse. To the extent one can construct adequate analytical models for the seismic source, path wave-propagation effects, and site nonlinear response, it becomes possible to simulate ground motion for geologic environments, distance ranges, and earthquake magnitudes for which data is scarce or nonexistent. 
The SH component of rock-site ground motion at distances 5 , 10, and 30 kilometers was synthesized for an earthquake model which consisted of an assemblage of discrete, circular cracks, representing a composite, extended source. It was found that strong motion observations are, in general, inconsistent with a rupture mechanism in which crack growth stops instantaneously. Once more realistic rupture deceleration was incorporated into the model, the SH component of motion alone was apparently adequate for simulating ground motion consistent with observed peak acceleration, peak velocity, and strong motion duration.

A new nonlinear constitutive model for soils, the endochronic model, was fit to cyclic shear data for dry sand, and an equivalent linear constitutive model was fit to the same sand data. Using the endochronic model, close agreement was achieved between calculated (solid curves) and observed (dashed curves) stress-strain behavior of dry sand over 300 cycles of deformation, as illustrated below.
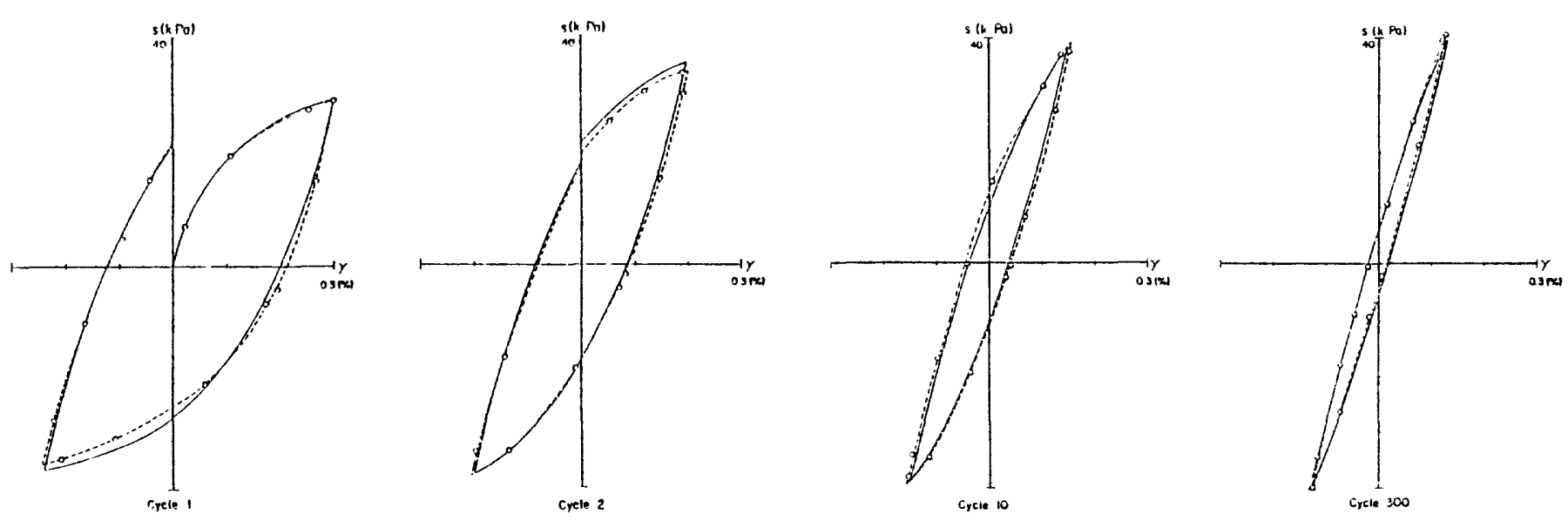

Only-five parameters were required to accomplish the fit to the sand data.

The nonlinear endochronic constitutive model and the base-rock synthetic seismograms were employed to calculate ground motion at sites on dry sand deposits, using a transient finite element method, and these calculations were repeated with the equivalent linear constitutive model, using the SHAKE code. The endochronic constitutive model performed effectively in the finite element code under the complex loading histories provided by the synthetic seismograms, and it was verified that (i) loading and unloading always in itiate along the elastic slope, (ii) all hysteresis loops close, (iii) the model does not display the unrealistic "corners" displayed by Iwan-type constitutive models when a loading or unloading curve intersects a previous loading or unloading curve, (iv) appreciable strain hardening is predicted for some loading histories, and (v) cyclic creep, or ratcheting, develops under asymmetric loading.

The predicted ground motion obtained from the nonlinear calculations is consistent with empirical trends governing peak motion where the trends are well-established by earthquake data. In the 
near-field, where bedrock acceleration peaks characteristically exceed 0.1 to $0.2 \mathrm{~g}$, nonlinear behavior sharply reduces the value of peak soil acceleration, and the equivalent linear method did not reproduce this effect. Earthquake data are consistent with this sharp reduction in the near-field, as summarized in the following figure comparing calculated peak accelerations for a dry sand deposit with empirical peak acceleration relations deduced by Seed et al.

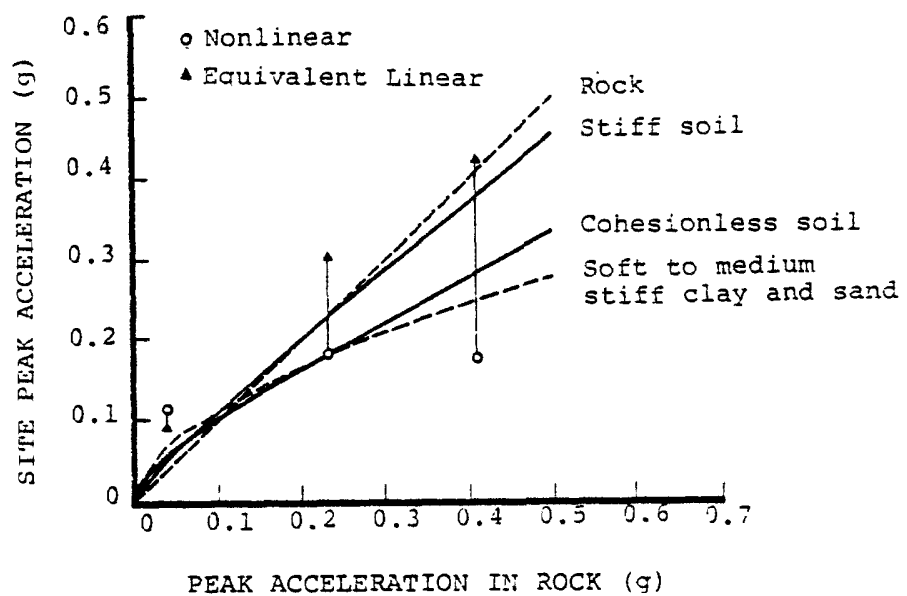

The performance of the equivalent linear approximation for the response of cohesionless soil depends on both the nature of the soil deposit and the amplitude level of the base-rock acceleration. Peak motion estimates by the equivalent linear method are adequate for peak input accelerations less than 0.1 to $0.2 \mathrm{~g}$, but are excessively conservative for stronger input accelerations.

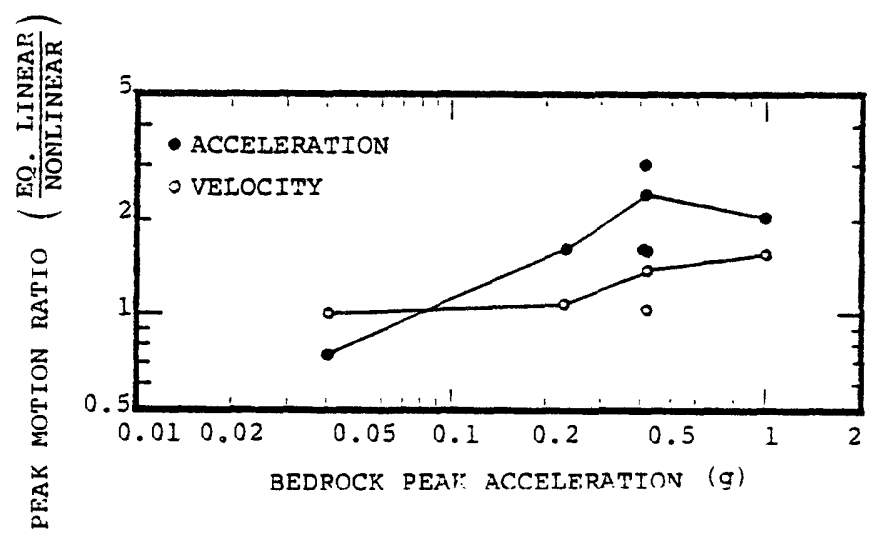

The overestimate of peak motion by the equivalent linear method is apparentiy due to an inability of the method to adequately model energy loss in the uppermost several meters of the soil column. 
TABLE OF CONTENTS

Page

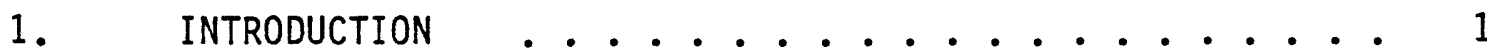

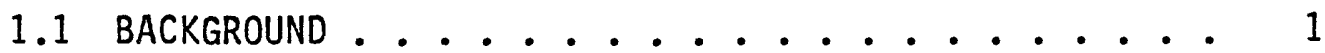

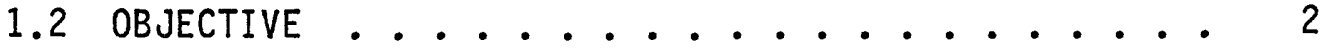

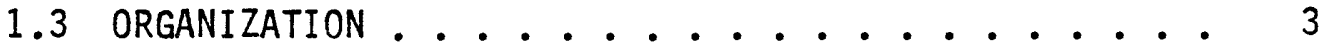

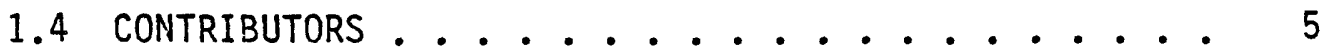

2. ROCK SITE GROUND MOTION SIMULATION ......... 7

2.1 INTRODUCTION ................... 7

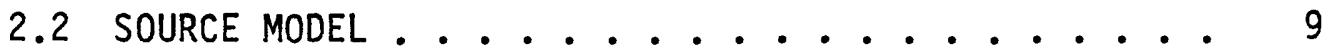

2.3 CIRCULAR SHEAR CRACK REPRESENTATION. ....... 11

2.4 THE SH-WAVE RESPONSE OF LAYERED EARTH MODELS . . . . 22

2.5 INCIDENT ANGLE DECOMPOSITION . . . . . . . 23

2.6 SYNTHETIC ROCK SITE MOTIONS . . . . . . . . 26

2.7 SUMMARY AND CONCLUSIONS . ......... 36

3. SITE SOIL MODELS ............... 39

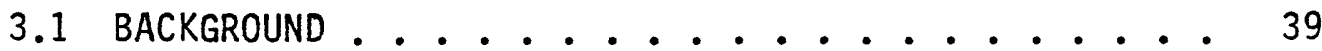

3.2 THE ENDOCHRONIC SOIL MODEL ............ 41

3.2.1 Endochronic Model for Simple Shear
of Sand ....... . . . . . . 4 43

3.2.2 Application of Model to Cyclic Data
for Sand....................... 46

3.3 THE EQUIVALENT LINEAR MODEL $\ldots \ldots 48$

4. SOIL SITE GROUND MOTION ............... 57

4.1 INTRODUCTION ...................... 57

4.2 DESCRIPTION OF THE NONLINEAR NUMERICAL STUDIES . . . 58

4.2.1 Soil Profiles Employed ......... 58

4.2.2 The Input Ground Motion ......... 60

4.3 NUMERICAL METHODS ................ 63 
4.3.1 Nonlinear Calculations Using SWIS . . . . 64

4.3.2 Equivalent Linear Calculations with SHAKE . . 67

4.4 NUMERICAL RESULTS ............... 69

4.4.1 Performance of the Endochronic Constitutive

Model ............... 73

4.4.2 Sensitivity to Soil Profile Details . . . . 81

4.4.3 Comparison of Peak Motion Estimates

with Empirical Trends......... 34

4.4.4 Comparison of Nonl inear and Equivalent

Linear Solutions ......... 89

5. SUMMARY OF RESULTS. .............. 101

REFERENCES . ..................... 107

APPENDIX A CALCULATION OF LAYERED MEDIUM SH-WAVE RESPONSE . . . 115

APPENDIX B DEPENDENCE OF MATERIAL PARAMETERS ON SOIL

DEPTH. .................... 123

B-1 SHEAR MODULUS ................ 123

B-2 SHEAR STRENGTH . . . . . . . . . . 127

B-3 EqUIVALENT LINEAR SHEAR MODIILUS ....... 130

APPENDIX C COMPUTER SUBROUTINE FOR SIMPLE SHEAR . . . . . . 135

APPENDIX D THE NEW ENDOCHRONIC SOIL MODEL: AN ASSESSMENT

OF ITS PRACTICAL UTILITY FOR USE IN TWO- AND

THREE-DIMENSIONAL GROUND MOTION CODES. . . . . 139

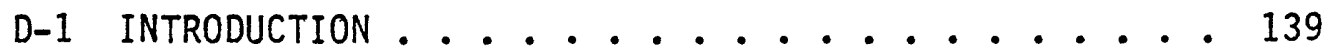

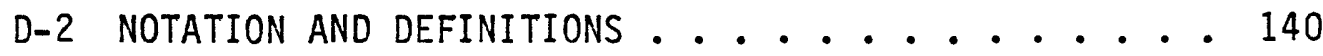

D-3 BASIC EQUATIONS OF NEW ENDOCHRONIC THEORY. . . . . 141

D-4 SOURCE OF COMPUTATIONAL DIFFICULTIES . . . . . 143

D-5 RECENT DEVELOPMENTS ................. 146 


\section{INTRODUCTION}

\subsection{BACKGROUND}

For distances in the range 20 through 200 kilometers, where the majority of the earthquake ground motion records have been obtained, the empirical relations used to predict likely characteristics of ground motion important for earthquake-resistant design agree to within a factor of two with the mean trends of the available data. For distances less than 20 kilometers, which are of the greatest interest to the seismic community, there are minimal ground motion data, and substantial disagreement exists between the empirical relations, reflecting the uncertainties associated with extrapolation of these empirical relations to shorter distances. There is some evidence also that the empirical relations, as a group, may be quantitatively inaccurate for short distances. In a recent study, Trifunac and Brady (1976) concluded that most previous investigators have underestimated peak acceleration for distances less than about 40 kilometers. The implications of such uncertainties on structural safety and human life are enormous. In view of the absence of significant ground motion data in the range of greatest structural concern, and the divergence among the extrapolated empirical relations in this range, there is clearly a critical need to develop reliable analytical methods that can be used in seismic design to predict the likely features of strong motion at distances relatively close to the earthquake focus.

The damage potential of an earthquake appears to be most likely determined by several characteristics of the ground motion, including the maximum velocity, maximum acceleration and the response spectrum. Observations made after destructive earthquakes have revealed a strong relationship between structural damage and local geology (see e.g., Seed and Schnabel, 1972; Schnabel, Seed and Lysmer, 1972); in general, destruction is larger on soft soils than on hard soils or rocks. Furthermore, observed damage patterns and 
statistical analyses of recorded ground motion suggest that both the stiffness and the depth of the soil deposit are important considerations in the design of earthquake-resistant structures. Response spectra also appear to be significantly dependent on local geology (Newmark, et. al., 1972; Seed, et. al., 1976b; Mohraz, 1976).

The analytical tool predominantly used at the present time to analyze seismic ground motion is the one-dimensional equivalent linear method, originally proposed by Idriss and Seed (1968). In this method, the soil medium is idealized as a series of thin horizontal soil layers capable of transmitting only shear stresses. An approximate one-dimensional constitutive model (termed an "equivalent linear" model) is employed to describe the mechanical response of the thin soil layers to vertically traveling shear waves driven by bedrock undergoing horizontal motion.

The appropriate bedrock motion is usually inferred from the recorded motion of nearby rock outcrops, if available, or simply assumed. Recordings made on nearby rock outcroppings may, or may not, be representative of the motions in the bedrock underlying the soil deposit (Lysmer, Seed and Schnabel, 1971); the motion at depth can only be inferred using some computational model. Furthermore, such recordings provide suitable input for two- or three-dimensional site analysis only if we prescribe a priori the spatial character of the emergent wave field (e.g., vertically incident shear waves).

\subsection{OBJECTIVE}

The objective of this research program is to develop and evaluate a new analytical approach for estimating local site response to earthquake ground motion. The approach is based on (1) an assumed, yet realistic, physical model of the earthquake source and wave propagation path to the site, using theoretical seismogram methods to compute base-rock seismic motion at the site, (2) applying an advanced nonlinear constitutive model, the endochronic model, to describe the hysteretic response of local soil deposits, and (3) computing surface motion at the site using a transient, dynamic finite element method. To the extent one can construct 
adequate models for the seismic source, path wave propagation effects, and site nonlinear response, it becomes possible to simulate ground motion in geological environments for which no data exist. Furthermore, the analytical approach is structured to potentially provide a three-dimensional ground motion capability, i.e., the theoretical seismic method, the endochronic constitutive theory, and the transient finite element method each have been developed within a fully three-dimensional framework.

The current study is limited, by the scope of the effort, to two site geologies -- rock and cohesionless soil. The predictive capabilities of the approach are examined by comparing ground motion calculations for these two site geologies with (1) empirically derived ground motion trends and (2) ground motion simulations by the equivalent linear method. The main computational tools employed in the study are: (1) a wavenumber-integration method for computing synthetic near-field accelerograms, (2) a transient, dynamic finite element method for computing the nonlinear response of soils, and (3) the SHAKE seismic analysis code for computing soil response by the equivalent 1 inear method.

\subsection{ORGANIZATION}

Section 2 describes the construction of base-rock seismic motion for a simplified model of the earthquake source, using a theoretical seismogram method. The source model, assembled from several superposed, discrete events, is motivated by some simple observational and theoretical considerations about earthquakes. Propagation of the earthquake disturbance through a stratified, anelastic earth model is accomplished by a direct wavenumber integration technique which is outlined in Appendix $A$; only the $S H$ contribution is included in the study, and some consequences of this limitation are discussed. We also outline a procedure for approximating a two-dimensional representation of the base-rock ground motion; this procedure would provide convenient input for two-dimensional numerical simulations of local site response. 
Finally, we describe the synthetic ground motion which results from the earthquake model, at three rock sites at 5,10 , and 30 kilometers from the source.

Section 3 describes a new endochronic constitutive model for rate-independent, history-dependent response of soils. This new model can describe the important features of the response of soils to seismic-type loading, including hysteresis, hardening/softening, densification and cyclic creep (ratcheting). A numerical method is developed for the model to treat the case of simple shear. The model is fit to cyclic shear data for dry sand. Finally, a corresponding equivalent linear representation is derived from the same data used in fitting the endochronic model. Appendix $B$ discusses the dependence adopted in the present study of the soil model parameters on depth within the soil column.

Section 4 applies the constitutive models of Section 3 and the base-rock ground motions of Section 2 to compute soil site ground motion. Particular attention is accorded to the numerics associated with the simple shear (one-dimensional) endochronic model under complex loading histories; the resulting FORTRAN subroutine is listed in Appendix $C$. The performance of the endochronic model in the numerical wave propagation code is examined, as is the sensitivity of computed ground motion to details of the soil profile. Finally, results from the nonlinear ground motion calculations are compared with trends in ground motion data and to ground motion computed by the equivalent linear method.

The nonlinear soil site computations reported in Section 4 are one-dimensional. We had hoped to exercise the multidimensional capability of the analytical method by performing in addition a small number of nonlinear, two-dimensional simulations. However, in the course of the study, a numerical problem related to the computational difficulties posed by the new endochronic model in two- and three-dimensional deformations was identified. Numerous attempts to 
resolve this difficulty during the course of the work were unsuccessful. Most recently, however, a promising approach has been uncovered (see Appendix $D$ for further details) and efforts are pressentry underway to fully explore this.

1.4 CONTRIBUTORS

A number of $\mathrm{s}^{3}$ scientists contributed to the work described in this report. Section 2 describing the synthesis of rock site ground motion, was written by $H$. Swanger. Section 3, which describes the nonlinear and equivalent linear soil models, was written by H. E. Read and K. J. Cheverton. Section 4, describing the soil site calculations, was written by S. M. Day, with important contrbuttons from $K$. J. Cheverton.

5 (page 7 follows) 


\section{ROCK SITE GROUND MOTION SIMULATION}

\subsection{INTRODUCTION}

Traditionally, observed ground motion has been the major source of input for studies of site and engineering structurespecific effects, such as nonlinear soil response or soil-structure interaction problems. Use of actual recorded ground motion as input for site specific modeling assures realistic frequency content and duration for the particular circumstances under which the ground motion was recorded, and results from empirical studies are often used to modify the amplitudes of recorded motion for applications to distances and magnitudes for which no strong ground motion recordings are available.

There are some applications, however, where observed ground motion and empirically motivated extrapolations of amplitudes may not be sufficient for describing input ground motion with proper charcteristics. Examples of circumstances are:

(1) Very short distances, where data are sparse and empirical extrapolation is uncertain.

(2) Very large earthquakes where motion may have larger amplitudes and longer durations than previously recorded ground motion.

(3) Response at the frequencies of interest may depend strongly on the rock structure with depth (i.e., long periods).

(4) Response is sensitive to two- or three-dimensional propagation effects, which cannot be extracted from ground motion recordings alone.

In such circumstances an alternative to observed ground motion is synthetic ground motion. Synthetic ground motions most often applied in engineering applications involve generation of a random signal which possesses a prescribed duration and spectral shape (Housner and Jennings, 1964, for example). Such procedures have evolved considerably to include more of the physical phenomena 
associated with time histories of strong motion accelerograms (Wong and Trifunac, 1979, for example). This "engineering" approach to construction of synthetic ground motion still depends strongly on empirical results, namely observed peak values and durations, which may not be adequate in all circumstances.

An alternative approach is to assume physical models for the earthquake source and propagation of seismic waves through the earth and compute the corresponding ground motion which is a consequence of the physical model assumed. This "seismological" approach to the problem, in theory at least, has several benefits over the engineering approach. If one can construct reasonable models for the seismic source and propagation effects, one can justifiably simulate ground motion in geological environments for which no data exist, and perhaps compute motion for close distances and large magnitudes which is more reliable than extrapolation of observed motion. In addition, effects due to directionalized rupture and source radiation pattern, which are not included in empirical relationships, can be dealt with rigorously.

In practice, the seismological approach to synthesis of design motion has many problems. Our knowledge of the earthquake source is rather limited as to details important to the high frequency (1 through $10 \mathrm{~Hz}$ ) radiation. It has been suggested that localized features, such as stress concentrations (Hanks, 1974; McGarr, et al., 1979) or fracture barriers (Aki, 1979), may control the high frequency radiation in the near field. Such processes may be difficult to duplicate in any deterministic source model. The response of the earth is often difficult to model. Complete layered-medium response is usually costly to compute for high frequencies, and there are circumstances where plane layered earth models are not adequate, such as when topographic effects are important (Boore, 1972; Wong and Jennings, 1975). The uncertainties about the exact nature of the physical processes in earthquakes which make deterministic synthesis difficult are, of course, not 
accounted for in any empirical extrapolation procedure. What has yet to be determined is whether the uncertainties in the final results of the complicated process of deterministic modeling are comparable to or less than the uncertainties in using the simple process of empirical scaling.

There have been very few attempts to synthesize the characteristics of strong ground accelerations using conventional earthquake source models, and most previous studies employed a certain degree of artificial randomness in the source description to obtain time histories with observed characteristics (Del Mar Technical Associates, (1978 and 1979), for example). It has not been determined what particular elements of the earthquake source model are necessary for obtaining the frequency content and amplitude decay with distance commonly observed in near-field recordings of acceleration.

In this study we use synthetic rock site ground motions generated by a very simple source model. It has been found that the durations and decay of peak amplitudes with distance observed from moderate earthquakes can be modeled adequately. The source model used is a sum of discrete crack approximations lagged appropriately to simulate slightly incoherent rupture. An essential feature of the model is the suppression of the strong stopping phases usually predicted from conventional source models. The response of the earth was modeled using complete SH-wave layered-medium Green's functions. The 1966 Parkfield, California, earthquake was used as a qualitative guide in specifying the source model parameters and the layered earth structure.

\subsection{SOURCE MODEL}

There are a wide variety of source models available in the seismic literature which have been used in the synthesis of earthquake ground motions. Unfortunately, nearly all models available have demonstrated their usefulness only at relatively low frequencies $(<1 \mathrm{~Hz})$. A recurring theme in the seismic literature 
is that because of the complicated nature of observed strong motion accelerations, the earthquake source must be rather complicated on a small spatial scale. It has been argued that earthquakes must be inherentiy complicated to be consistent with observed trends in seismicity (Hanks, 1979; Andrews, 1979). These inferred complications have led several authors to construct stochastic models of the source details (Boore and Joyner, 1978; Del Mar Technical Associates, 1979; Kanamori, 1979).

Close examination of strong ground motion recordings and short-period teleseismic recordings suggests that the high frequency radiation in many earthquakes is controlled by a few discrete sources of energy associated with stress concentrations or fracture barriers, and that the dimensions of these sources are often considerably smaller than the overall source dimensions inferred from surface ground breakage, aftershock distributions, or teleseismic modeling. Some examples are the 1971 San Fernando, California, earthquake (Hanks, 1974; Bache and Barker, 1978; Bouchon, 1978), the 1975 Pocate110, Idaho, earthquake (Bache, et al., 1980), and the 1975 Horse Canyon, California, earthquake (Hartze11 and Brune, 1979).

Motivated by such observations, we constructed a model of a moderate earthquake consisting of a few small, discrete events. Each discrete event was assumed to be an independent release of prescribed stress. The dynamics of rupture do not physically permit truly independent stress-drop sources, since the release of stress of one source will change the stress field of the others. However, the results of numerical studies by Day (1979) employing a fracture criterion and spontaneous rupture suggest that superposition of such sources may be a reasonable approximation in many cases.

The radiation from each discrete event in the model is represented by a geometrical far-field approximation (Fraunhofer approximation) of the radiation due to a simple dynamic shear crack embedded in a wholespace, convolved with the point-source response (Green's function) of the layered medium. The crack approximation 
to the fault slip history is described below. The wholespace radiation from a shear crack is, or course, takeoff-angle (or wavenumber) dependent, and for simplicity we assume that the appropriate far-field source-time function is that corresponding to the direct S-wave arrival. For all cases in this study, the source is located in the uniform half-space underlying the layered model, and most of the high frequency energy arrives at times very near the direct wave arrival.

\subsection{CIRCULAR SHEAR CRACK REPRESENTATION}

Several closed-form analytic approximations to the slip history of a circular shear crack with uniform stress-drop are available. These are generally motivated by the early analytical solution of Kostrov (1964) and numerical simulations, like Madariaga (1976), which include the stopping of rupture. Boatwright (1980) provides a summary of available analytic expressions. Even though simple expressions for the slip history on the fault exist, simple representations of the geometrical far-field radiation, in general, do not. The exception is the model of Sato and Hirasawa (1973).

The Sato and Hirasawa model (which we will call the S \& H model.) is simply an expanding, constant rupture velocity, circular crack for which slip everywhere on the crack terminates instantaneously when the rupture reaches a prescribed radius. Their model is not a rigorous dynamic solution, as shown by the numerical solution of Madariaga (1976). His calculations revealed that termination of slip actually occurs only when healing phases propagate inward from the fault edge at the $P$ and $S$ velocities of the medium. The advantage of the $S$ \& $H$ model is that its geometrical far-field radiation can be obtained analytically in closed form. Given the geometry shown in Figure 2.1, the geometrical far-field acceleration can be written 


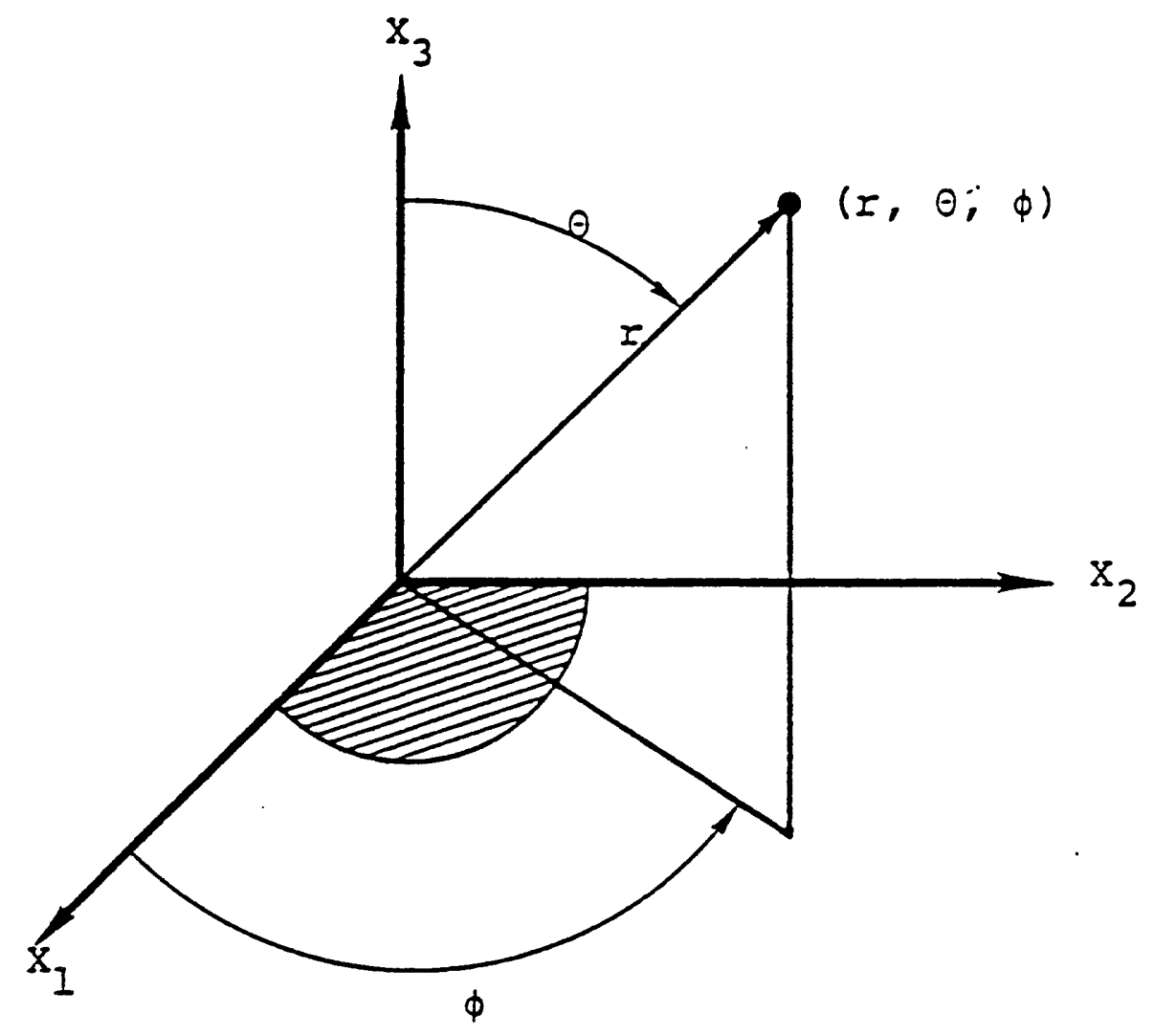

Figure 2.1. Coordinate system conventions used. Shaded area represents the fault surface. 


$$
\begin{aligned}
& \ddot{u}_{r}=\frac{(\beta / \alpha)^{3}}{4 \pi \beta r} \sin 2 \theta \cos \phi \ddot{I}_{\alpha} \\
& \ddot{u}_{\theta}=\frac{1}{4 \pi \beta r} \quad \cos 2 \theta \cos \phi \ddot{I}_{\beta} \\
& \ddot{u}_{\phi}=\frac{-1}{4 \pi \beta r} \quad \cos \theta \sin \phi \ddot{I}_{\beta}
\end{aligned}
$$

with

$$
\begin{aligned}
\ddot{I}_{C} & =\frac{4 \pi \dot{D}_{0} V^{2}}{\left(1-K^{2}\right)^{2}}\left[H(t)-H\left(t-\frac{L}{V}(1-K)\right)\right] \\
& -\frac{4 \pi \dot{D}_{0} V^{2}}{K(1+K)^{2}}\left[H\left(t-\frac{L}{V}(1-K)\right)-H\left(t-\frac{L}{V}(1+K)\right)\right] \\
& -\frac{\dot{D}_{0} V L}{K(1-K)} \delta\left(t-\frac{L}{V}(1-K)\right) \\
& +\frac{\pi \dot{D}_{0} V L}{K(1+K)} \delta\left(t-\frac{L}{V}(1+K)\right)
\end{aligned}
$$

where

$$
\begin{aligned}
& D_{0} \quad=\text { slip velocity at center of crack } \\
& V \quad=\text { rupture velocity } \\
& L \quad=\text { fault radius } \\
& K \quad=V / C \sin \theta \\
& \mathrm{L} \quad=\text { reduced time. }
\end{aligned}
$$




$$
\begin{aligned}
\alpha, \beta= & \text { compressional and shear velocities, respectively in } \\
& \text { the source region, } \\
c \quad= & \alpha \text { or } \beta \\
H(t)= & \text { Heaviside step function } \\
\delta(t)= & \text { delta function }
\end{aligned}
$$

The time domain behavior is shown in Figure 2.2. Acceleration initiates as a step which continues until the arrival of the stopping phase from the edge of the fault nearest the observer. From that time until the time of arrival of the stopping phase from the farthest edge of the fault, the acceleration is constant.

There are several noteworthy characteristics of the accelerations. The high frequency content is clearly dominated by the stopping phases, which are (mathematically) delta functions. These cause the far-field displacement spectrum to decay as $\omega^{-2}$ at high frequencies. The amplitudes of the first stopping phase and the step initiating the motion contain terms of $K(1-K)^{-1}$ and $\left(1-k^{2}\right)^{-2}$ respectively. These terms make the amplitudes of the early parts of the motion rather strong functions of the rupture velocity and the azimuth of the observer from the fault normal. For example, with a rupture velocity of $0.9 B$, where $B$ is the shear speed of the medium, the amplitudes of the initiation phase in the plane of the fault and at the fault normal differ by more than a factor of 27. For a given slip velocity, changing the rupture velocity from $0.8 \mathrm{~B}$ to $0.9 \mathrm{~B}$ causes the initiation phase to increase by a factor 4.5 in the plane of the fault, but only by 1.1 at the fault normal.

Sato and Hirasawa motivated their model with a static interpretation of the source displacement field shape and amplitude. Consequently, the relationship between stress drop and slip velocity at the crack center is given as

$$
\dot{D}_{0}=\frac{24}{7 \pi} \vee \frac{\sigma}{\mu} \approx 1.1 \vee \frac{\sigma}{\mu}
$$




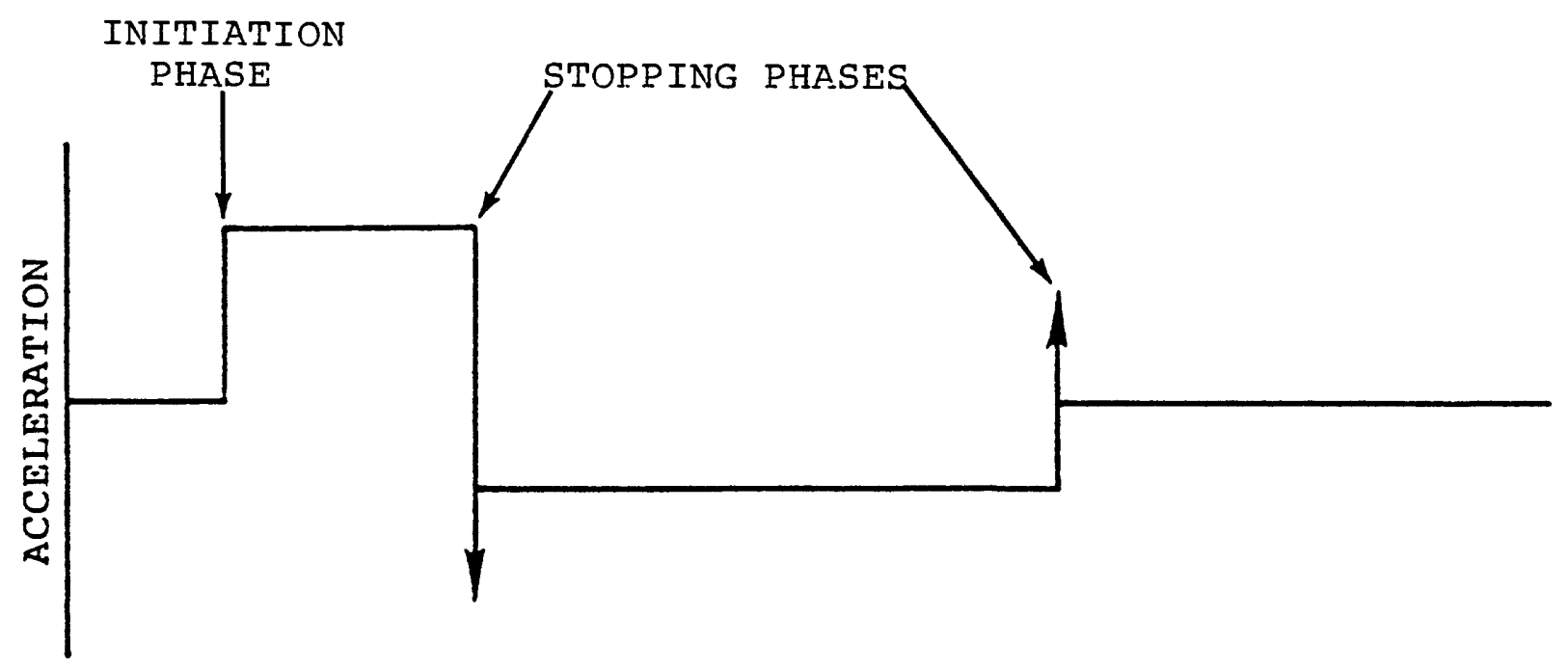

TIME

Figure 2.2. Typical shape of the Sato and Hirasawa model geometrical farfield acceleration. Arrows represent delta functions. 
where $\sigma$ is the stress drop and $\mu$ the medium shear modulus.

A rigorous expression using dynamic considerations was derived by Kostrov (1964) and computed by Dahlen (1974). For rupture velocities near $B$, the dynamic results suggest a better approximation to be

$$
\dot{D}_{0} \approx 0.8 \vee \frac{\sigma}{\mu}
$$

and this relationship is used here.

The delta-function dependence in the stopping phase will, of course, be smoothed by an attenuating medium, but even with reasonable values for the intrinsic attenuation $Q^{-1}$, it appears that the predicted stopping phases are much too strong to be consistent with near-field observations. The amplitude of the phase can be roughly estimated, given linear attenuation, using the asymptotic formulas of Kjartansson (1979). He suggests the peak time domain amplitude of a causal, attenuated pulse to be roughly $C Q / R$ times the strength of the input delta function, where $C$ is the signal velocity and $R$ is the distance traveled. For example, Hanks (1974) suggested that the initiation event for the 1971 San Fernando, California, earthquake had a stress drop of 350 to 1400 bars over a fault radius of 3 to 6 $\mathrm{km}$. We can estimate the relative peak amplitudes of the initiation and stopping phases observed at Pacoima Dam, $R=15$ kilometer and very near the plane of the fault. Figure 2.3 shows the values predicted by the $S \& H$ model for a 400 bar stress drop and 5 kilometer fault radius, for a 0 of 100 . Except when the rupture velocity is near the shear velocity, the stopping phase is estimated to be considerably larger than the initiation phase. only relatively slow rupture velocities can predict reasonable values. Since the absolute values depend on many poorly constrained parameters, the relative values are the most meaningful. If actual recordings are examined (Figure 2.4), the step-like feature is evident on all three components of motion about $2.5 \mathrm{sec}$ into the 


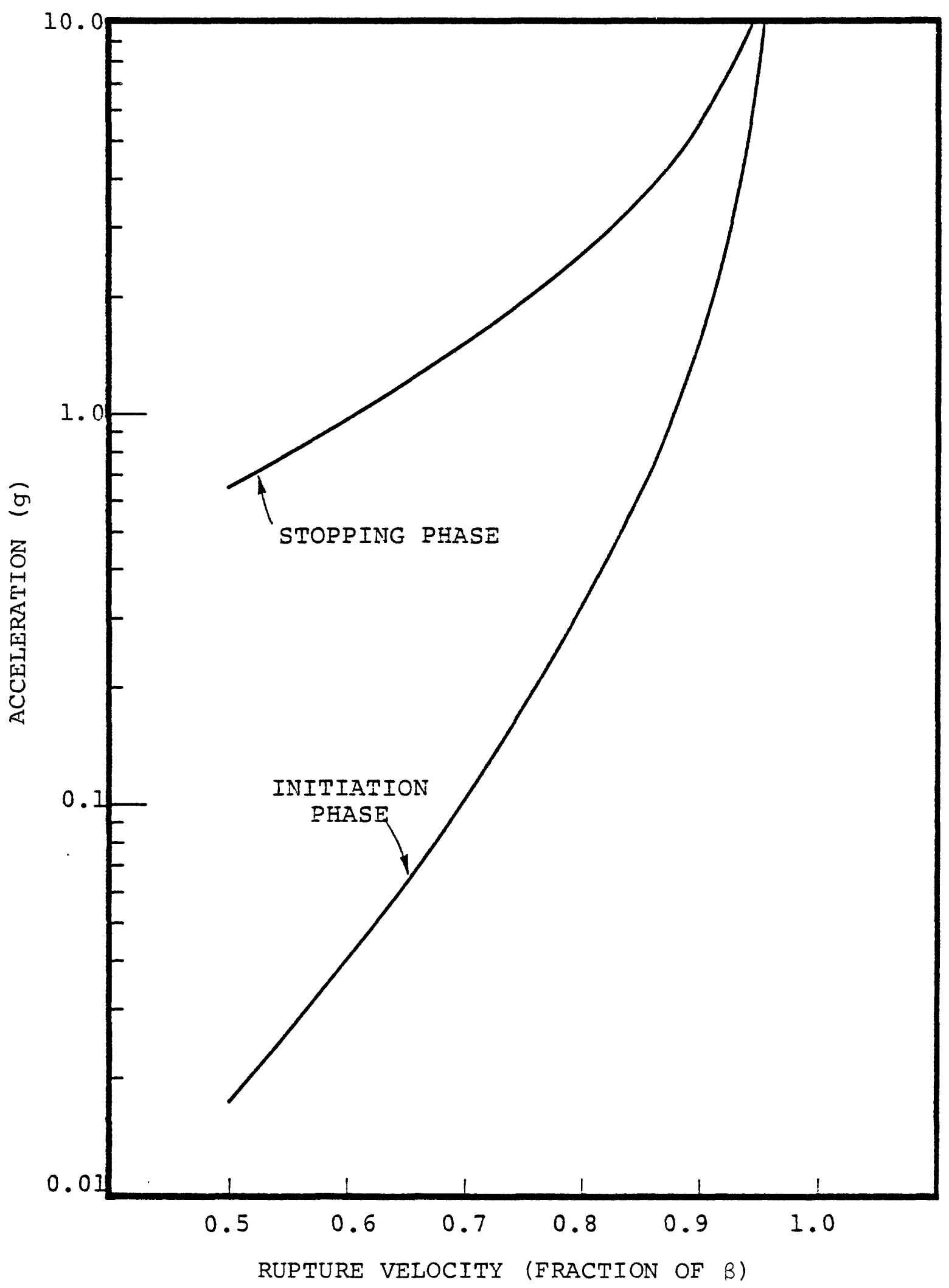

Figure 2.3. Initiation-phase and stopping-phase amplitude as predicted by the $\mathrm{S} \& \mathrm{H}$ model for a 400 bar stress drop and $5 \mathrm{~km}$ fault radius, assuming a $Q$ of 100 . 

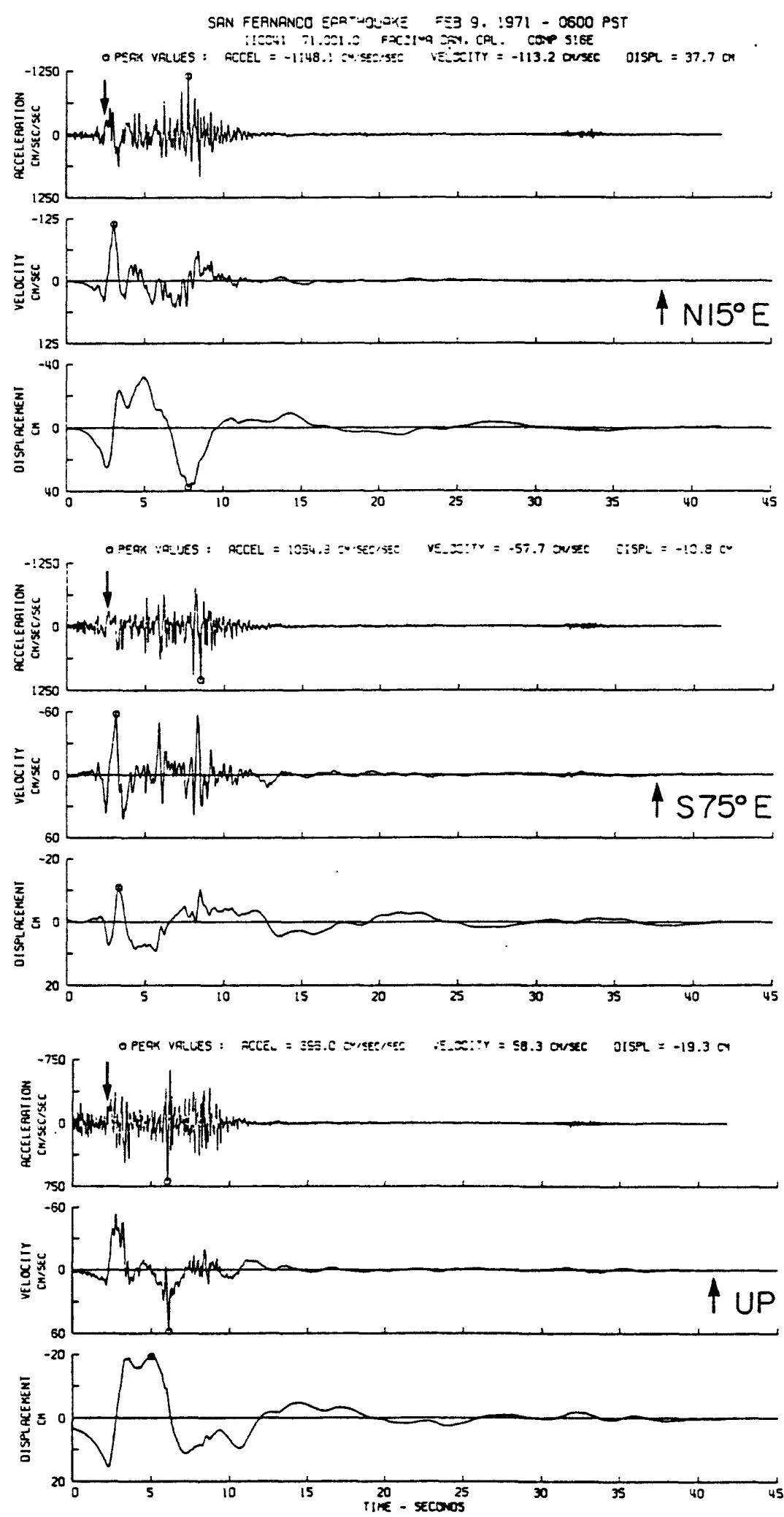

Figure 2.4. Processed ground motion observed at Pacoima Dam. Arrows point out the step-like initiation phases on acceleration records (modified from Heaton and Helmberger, 1979). 
record. After approximately $0.6 \mathrm{sec}$, there is a sudden downward phase indicating some kind of stopping, but the peak amplitudes are at best comparable to the initiation phase, on the order of $0.5 \mathrm{~g}$. A better evaluation of the model is achieved by using the stopping time $(t=0.6 \mathrm{sec})$ to constrain the fault radius $L=v t /(1-K)$. When this is done, the predicted stopping phase is 7 to 8 times larger than the predicted initiation phase for all rupture velocities between $0.5 \beta$ and 0.998 .

Clearly, the stopping phases of the $S \& H$ model are much too strong, in a linearly attenuating medium, to be reasonable. There are numerous explanations for this inadequacy of the model, and a detailed discussion will not be undertaken. There are two items worth discussing -- the mode of healing and the abruptness with which rupture growth terminates. The $S \& H$ solution does not treat healing rigorously. Numerical solutions suggest that the healing of the fault when rupture propagation terminates is not instantaneous, as assumed by the $S \& H$ model, but propagates inward at the seismic signal velocities. The approximate treatment of healing is probably not as influential in controlling peak acceleration as it may at first seem; Madariaga's (1976) numerical solution, which treats healing more rigorously, has an $\omega^{-2}$ far-field displacement spectrum indicating that it, too, gives singular acceleration.

Of greater importance is the manner in which rupture growth stops. In the $S \& H$ model, rupture stopping is very idealized. The propagating rupture decelerates instantaneously along a smooth, prescribed boundary (a circle). The values of observed peak accelerations suggest that this approximation is unacceptable for predicting the characteristics of high frequency radiation.

Clearly, a decelerating model of rupture is needed. The D-model of Boatwright (1980) would be an appropriate choice, but at present no simple far-field representation of the motion from this model is available. Although an analytic far-field solution is not essential, it does add considerable flexibility to the calculations 
and facilitates interpretation of computed waveforms. As an alternative to employing a rigorous numerical solution for a decelerating rupture model, we choose, for simplicity, to alter the form of the far-field radiation from the $S$ \& $H$ model to have a deemphasized stopping phase. The most convenient way to do this is to assume that the rupture decelerates over an annular region of width $S$. The stopping phase will then be smoothed out over a time proportional to S. For simplicity we use a smoothing function $F$, where

$$
F=\frac{V}{(1 \mp K) S}\left[H\left(t-\frac{S(1 \mp K)}{2 V}\right)-H\left(t-\frac{S(1 \mp K)}{2 V}\right)\right](2-4)
$$

where $\mp$ refers to the first and second stopping phases respectively. The delta functions become boxes whose height and duration depend on the source-receiver direction relative to the fault normal. This modification provides the desired effects at the cost of introducing a new parameter, $S$. The modified time domain far-field acceleration is shown in Figure 2.5. Note that the transition from the positive to negative steps is also smoothed, causing the stopping phase to be trapezoidal. Attenuation of the medium will further smooth the sharp features of motion.

In summary, the Sato and Hirasawa crack approximation appears to be unreasonable when high frequency radiation is of interest. The large stopping phases predicted by the model are, in general, too large to be consistent with observed values of acceleration close to earthquakes. A more acceptable model for the radiation from a stress concentration apparentiy requires a deemphasis of the high frequency radiation due to the stopping of rupture. Here we choose an alternative representation of the far-field radiation from an isolated release of stress which includes a smoothing of these stopping phases. 
$S$ AND H MODEL

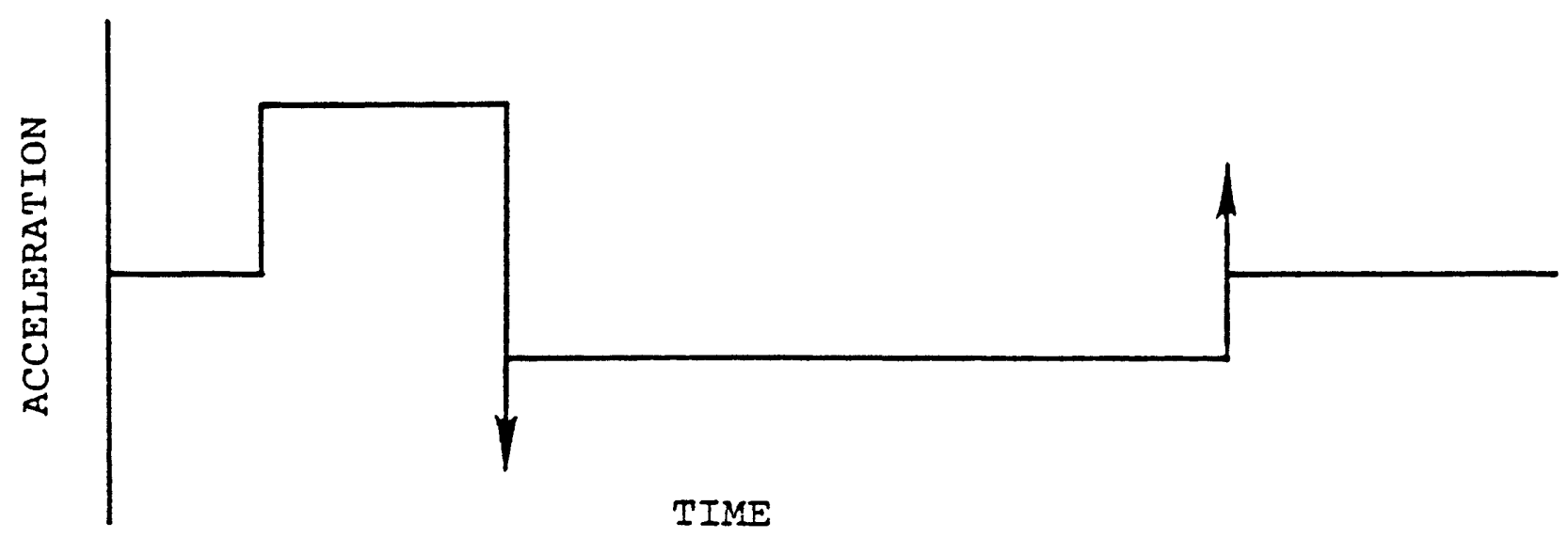

MODIFIED MODEL

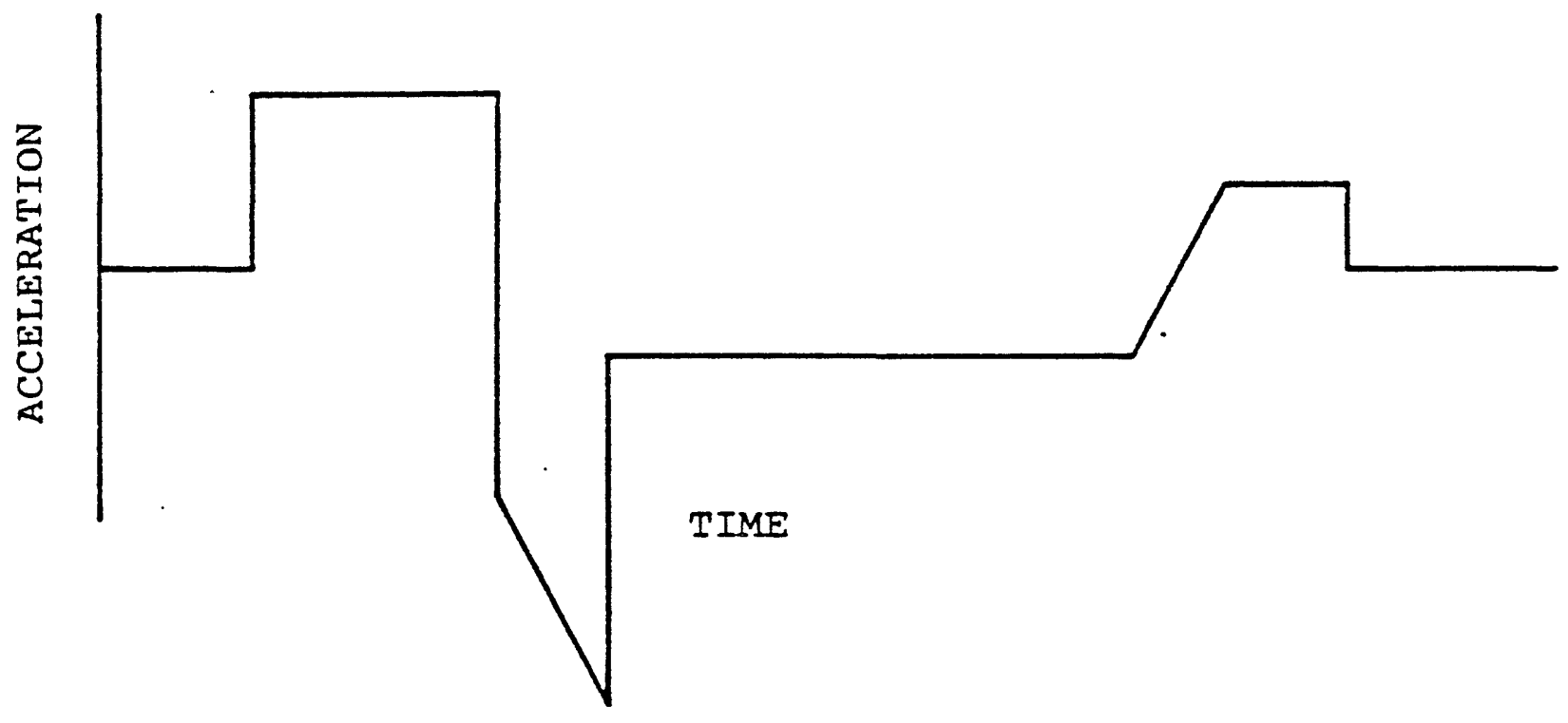

Figure 2.5. Comparison of typical shapes of far-field acceleration from the Sato and Hirasawa model and the modified model used in this study. 


\subsection{THE SH-WAVE RESPONSE OF LAYERED EARTH MODELS}

The response of rock site geologic structure is approximated by the complete SH-wave response for a linearly viscoelastic, plane-layered halfspace, computed by a direct wavenumber integration method. The procedure used is quite similar to that of Apsel (1979). The numerical procedures are described more completely in Appendix A.

The precise procedure differs from Apsel (1979) in only a few details. A complex frequency is introduced to permit equal-step sampling of the Fourier-Bessel integrals, an approach similar to that of Bouchon (1978). The total SH motion for a horizontal point force at the surface is computed within the halfspace by a recursion on the coefficients of up- and down-going waves. Reciprocity relationships are then used to obtain the surface response to any buried point force or point dislocation. The causal, frequency-independent $Q$ operator of Kjartansson (1979) is used in each layer to model anelastic attenuation.

The use of discrete sources described earlier makes the use of layered medium response more practical than if a coherent rupture response were desired. To model coherent rupture, one needs a very dense sampling of the Green's function, which can be quite costly to compute. Here we use at most eight Green's function evaluations per seismogram.

The classification "rock site" is reserved for sites which have no, or very thin, soil profiles. One often assumes that the particulars of the rock site have little to do with determining the amplitudes and frequency content of the surface motion. This is certainly not true for the long periods (1 through 10 seconds), (Swanger and Boore, 1978). In the present study we have found that rather innocent looking layered structures can also cause amplification of the high frequencies over the uniform half-space response. The amplitudes of the high-frequency arrivals are very complicated functions of depth and range. The behavior found here is quite similar to that found by DELTA (1978). 
The SH-wave response is, of course, not a complete response. Leaving out the P-SV motion will cause some problems. We would expect the seismograms to be somewhat simpler without the presence of high-frequency $P$ wave energy, which is not affected by attenuation as much as is $S$ wave energy. We would also expect SH nodes in the radiation pattern to be more obvious. In other words, contributions from rupture through an SH-node will leave a quiet zone in the record which would be filled in by P-SV motion in actual recordings.

\subsection{INCIDENT ANGLE DECOMPOSITION}

When a two- or three-dimensional description of the input motion is desired for computing near-surface soil response or soil-structure interaction, synthetic ground motion provides a natural description of the multi-dimensional characteristics of the motion. When layered medium solutions are computed in the frequency-wavenumber domain, an approximate separation by angle of incidence can be conveniently made before a final seismogram is computed.

Even though no two-dimensional soil response calculations were made in this study, an effort was made to decompose synthetic rock site motion by angle of incidence. Though this process appears straightforward in theory, it has some difficulties in practice. The difficulties arise because the time domain signal from a given ray-parameter (or angle of incidence), or a range of ray-parameters, is, in general, not causal (see Chapman, 1978). In the near-field there is also no guarantee that one can associate a given angle of incidence with a single horizontal wavenumber. This is most easily seen in the simplest of examples, a spherical wave in a whole space. In the time-space domain a spherical wave might be written

$$
u(R, t)=\frac{A}{R} \delta(t-R / C)
$$


where $c$ is the velocity of propagation and $A$ is a constant. In the frequency-space domain

$$
u(R, \omega)=A \frac{e^{-i \omega R / C}}{R}
$$

and in a cylindrical frequency-wavenumber domain

$$
\begin{aligned}
u(k, \omega) & =A \int_{0}^{\infty} \frac{k d k}{n} e^{-n h} J_{0}(k r) \\
n & =\left[k^{2}-\frac{\omega^{2}}{c^{2}}\right]^{1 / 2} \quad \text { Ren } \geq 0 \\
R & =\left[r^{2}+h^{2}\right]^{1 / 2}
\end{aligned}
$$

It is usualiy assumed that the horizontal wavenumber $k$ is related to the incident angle $\theta$ through the relationship

$$
k=\frac{\omega \sin \theta}{\mathrm{c}}
$$

Note that the representation of a wave which one would expect, at any observer point, to contain a single angle of incidence actually consists of an integral over all wavenumbers. It is only for large $\omega$ and $r$ that this integral will be dominated by a narrow range of $k$. It is clear that separation by angle of incidence through filtering in the $\omega-k$ domain may have problems at close distances.

As an example, we attempted a decomposition of a near-field Green's function by computing the response due to overlapping triangular wavenumber filters corresponding to various ranges of angles of incidence and assumed $k=\omega \sin \theta / \beta_{1}$ where $\beta_{1}$ is the shear velocity in the shallowest rock layer. Overlapping windows were used to avoid truncation effects. Figure (2.6) shows the complete seismogram and the seismogram components for 15 degree 


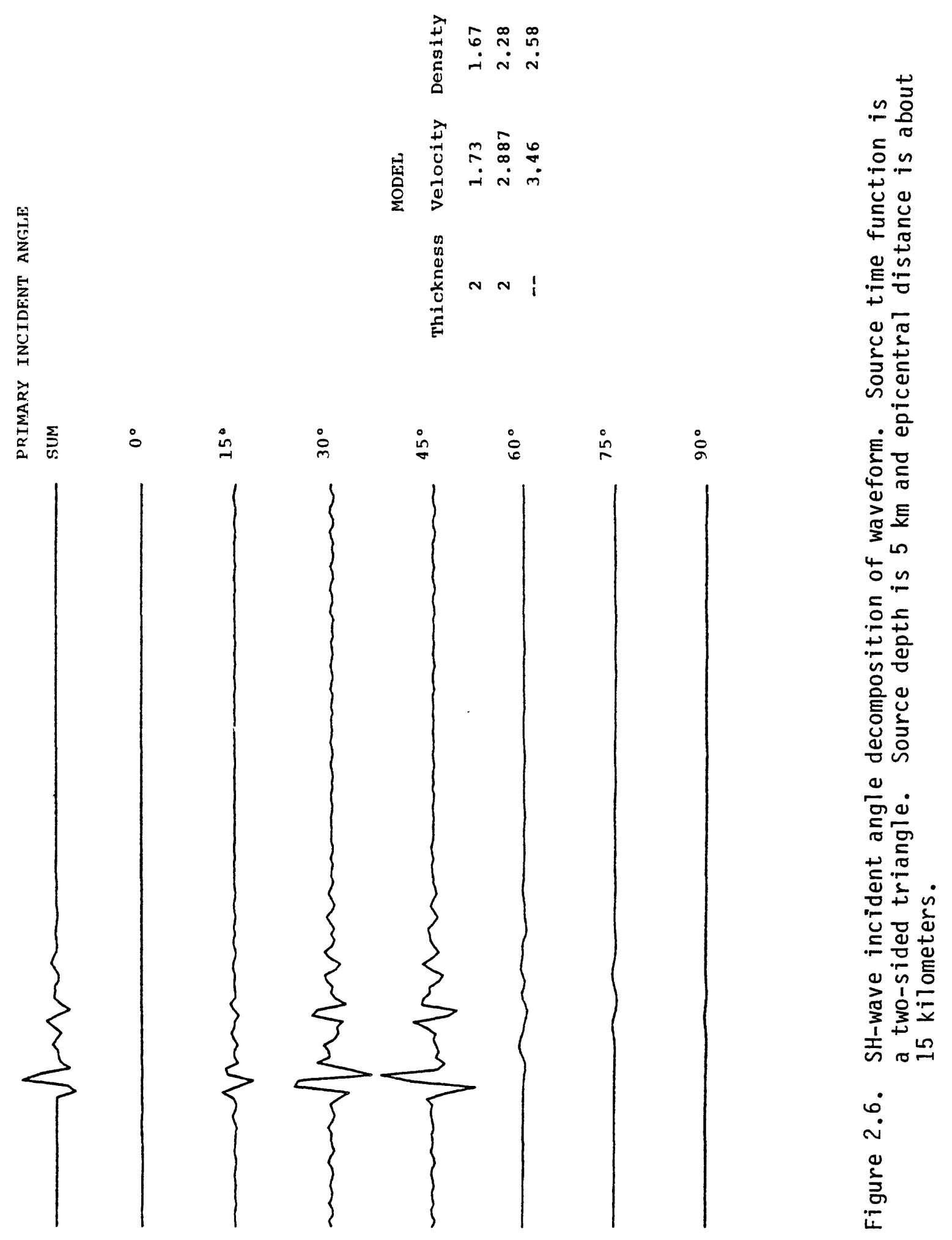


incident-angle windows. Note that the largest component is larger in amplitude than the complete seismogram, and considerable destructive interference occurs between bands. Although the above decomposition is approximate, it provides a convenient means for introducing realistic seismic motion into multi-dimensional nonlinear site simulations. It is clear that the input wave field spans a wide range of incidence angles, and the assumption of a single input angle can be quite poor.

\subsection{SYNTHETIC ROCK SITE MOTIONS}

In this section we describe the ground motions simulated using the individual elements described in the previous sections. For this purpose the 1966 Parkfield, California, earthquake is used as a guide to construction of a reasonable source model of a moderate sized event, and the near-field recordings of this event are used to verify the amplitudes and durations of the synthesized ground motions at close distance. We are not attempting to "model" the Parkfield ground motion recordings, but only trying to obtain qualitative agreement in amplitudes and durations of observed motion.

Details of the Parkfield event such as the fault length and the depth of rupture appear to be only partially understood, considering the range of model parameters which have been used to model the near-field displacement observations. Most studies suggest that rupture extended past Station Number 2 to the south, while some evidence suggests termination of rupture at Gold Hill, 10 kilometers to the north of Station Number 2 (Lindh and Boore, 1974). Bouchon (1979) suggests that the characteristics of motion recorded at Station Number 2 require very shallow rupture under Station Number 2 , including significant radiation coming from slip in the nearsurface sediments. Archuleta and Day (1980) obtained satisfactory fits to displacement histories at all stations using a much deeper rupture. It appears that the conclusions reached by these authors 
are strongly dependent on the type of source model assumed and on the manner in which the response of the near surface sediments is dealt with.

Here we assume a fault length of 34 kilometers and a fault width of 6 kilometers. The fault is divided into eight slightiy overlapping, discrete sources, each with diameter equal to the fault width. For simplicity we will assume that each source has identical properties except for time of initiation. The time of initiation is controlled by a "global" rupture velocity. The free parameters are the stress drop, "local" rupture velocity, the smoothing parameter, stopping, the global rupture velocity, and the source depth. These parameters were varied until the strong motion duration, maximum acceleration, and maximum velocity observed at Stations Number 5 and 8 were approximated.

Table 2.1 summarizes the source parameters used for the discrete sources. The constraint of fitting observed duration, peak acceleration, and peak velocity required a fast local rupture velocity $(0.9 \mathrm{~B})$ with a relatively deep source $(7.5$ kilometers $)$. The total moment of the eight sources is approximately 2 or 3 times larger than values normally quoted for Parkfield. This is to be expected since we are employing bi-directional rupture sources; the time domain contributions from these sources will be dominated by the rupture toward the observer and the rupture going away will affect only the long periods. The stress drop used is only a local stress drop; the area over which stress is released in this model is less than the total area which would be inferred assuming a rectangular fault surface containing all of the discrete sources.

Figure 2.7 shows the geometry of the source and receivers. The global rupture advances toward the stations, which are located on a line normal to the fault strike. 
TABLE 2.1

DISCRETE SOURCE PARAMETERS USED

Local and global rupture velocity

Coherent rupture radius

Smoothed rupture radius

Stress drop

Moment

Depth
$3.15 \mathrm{~km} / \mathrm{sec}$

$2.2 \mathrm{~km}$

$0.8 \mathrm{~km}$

95 bars

$7 \times 10^{25}$ dyne-cm

$7.5 \mathrm{~km}$ 


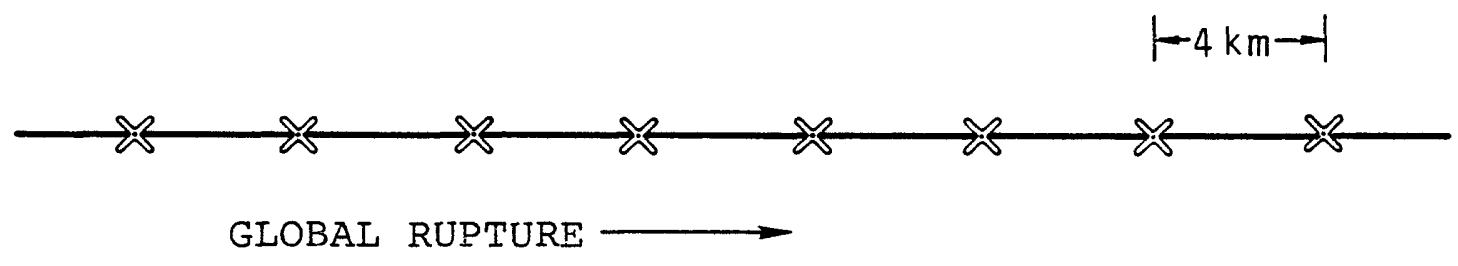

$\nwarrow=$ Discrete source location

- $5 \mathrm{~km}$

- =bserver locations

- $10 \mathrm{~km}$

- $30 \mathrm{~km}$

Figure 2.7. Source and receiver geometry. Receiver distances are measured from the surface projection of the fault plane. 
The layered structure used (Table 2.2) was taken from Anderson (1974). The layer shear Q's used were upper bounds of the observed values quoted by DELTA (1978).

The computed velocities and accelerations are shown in Figure 2.8. The motions shown are the parallel (with respect to the fault strike) component, which for all cases was larger than the perpendicular component. At the close stations, the largest motions are caused by the closest discrete source. At $30 \mathrm{kilometers} \mathrm{the}$ last few sources contribute almost equally.

The recording at 5 kilometers has a quiet period just before the onset of the largest motion. This is due to the global rupture passing through an SH-wave node. The second to last discrete source gives virtualiy no contribution because of this effect. This is not realistic, since P-SV waves would radiate significantly at these azimuths, and would fill in the quiet area. The point-source approximation of the local rupture also exaggerates the effect of the node in the SH-wave double-couple radiation pattern.

Local seismic directivity plays a strong role in determining the overall shape of the waveforms. At the 5 kilometer station the waveform from each discrete source is rather narrow. At more distant stations, the waveforms broaden. The superposition of wavelets of similar shape from the discrete sources leads to unusual looking seismograms. The discrete source model would probably give better results if the sources had the varied dimensions and rupture characteristics which would be inferred from a more detailed modeling of the data.

The response spectra from the three distances are shown in Figures 2.9, 2.10, and 2.11. The pseudo-velocity spectrum at 5 kilometers is somewhat differentiy shaped from these at the distant sites. The distant sites have peaked spectra, an effect which is probably related to the periodicity of arrivals from the discrete sources. The long period behavior observed is due to the smoothing performed to the $S$ \& $H$ far-field time histories. The smoothing 
TABLE 2.2

\section{LAYERED STRUCTURE}

\begin{tabular}{cccc}
$\begin{array}{c}\text { Thickness } \\
(\mathrm{km})\end{array}$ & $\begin{array}{c}\text { Shear } \\
\text { Velocity } \\
(\mathrm{km} / \mathrm{sec})\end{array}$ & $\begin{array}{c}\text { Density } \\
\left(\mathrm{gm}^{\mathrm{c}} \mathrm{cm}^{3}\right)\end{array}$ & $\begin{array}{c}\text { Shear } \\
0\end{array}$ \\
\cline { 2 - 3 } 0.28 & 0.98 & 2.10 & 50 \\
1.27 & 1.62 & 2.55 & 100 \\
2.19 & 2.89 & 2.63 & 200 \\
3.76 & 3.46 & 2.85 & 350
\end{tabular}


VELOCITY
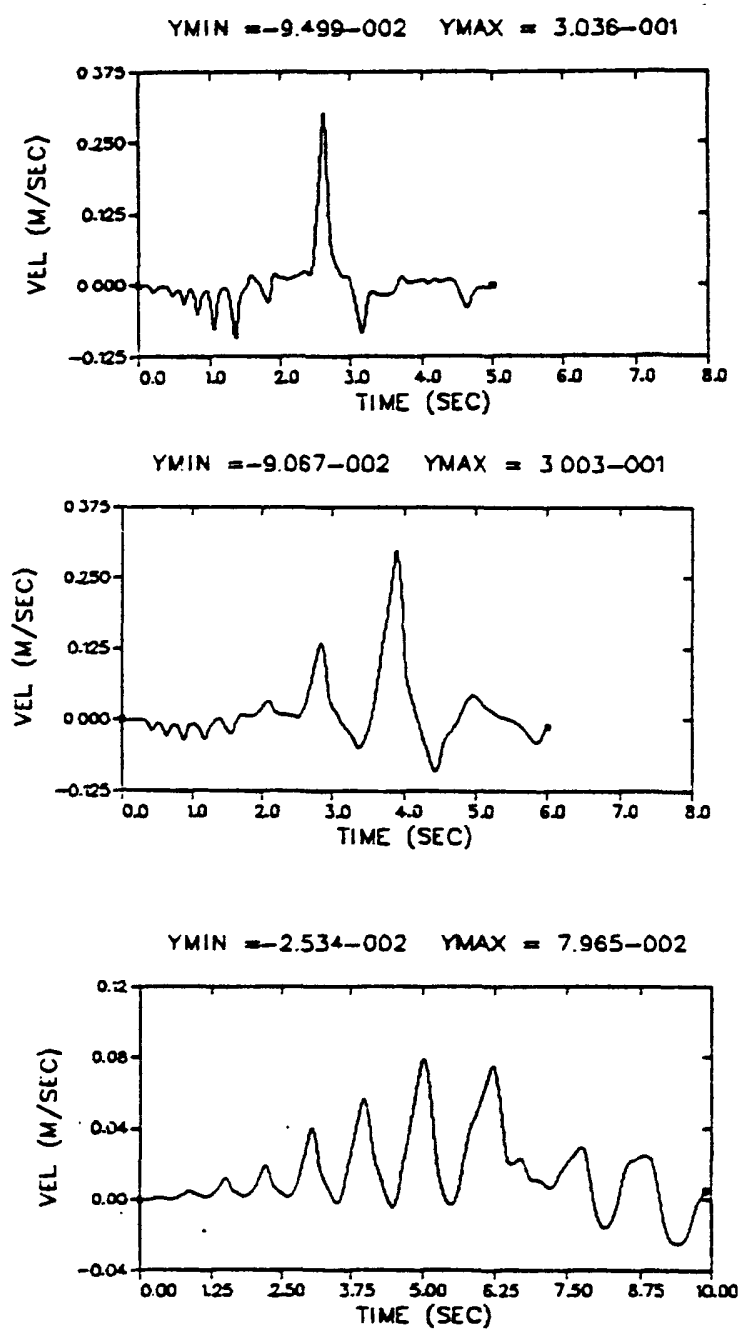

ACCELERATION
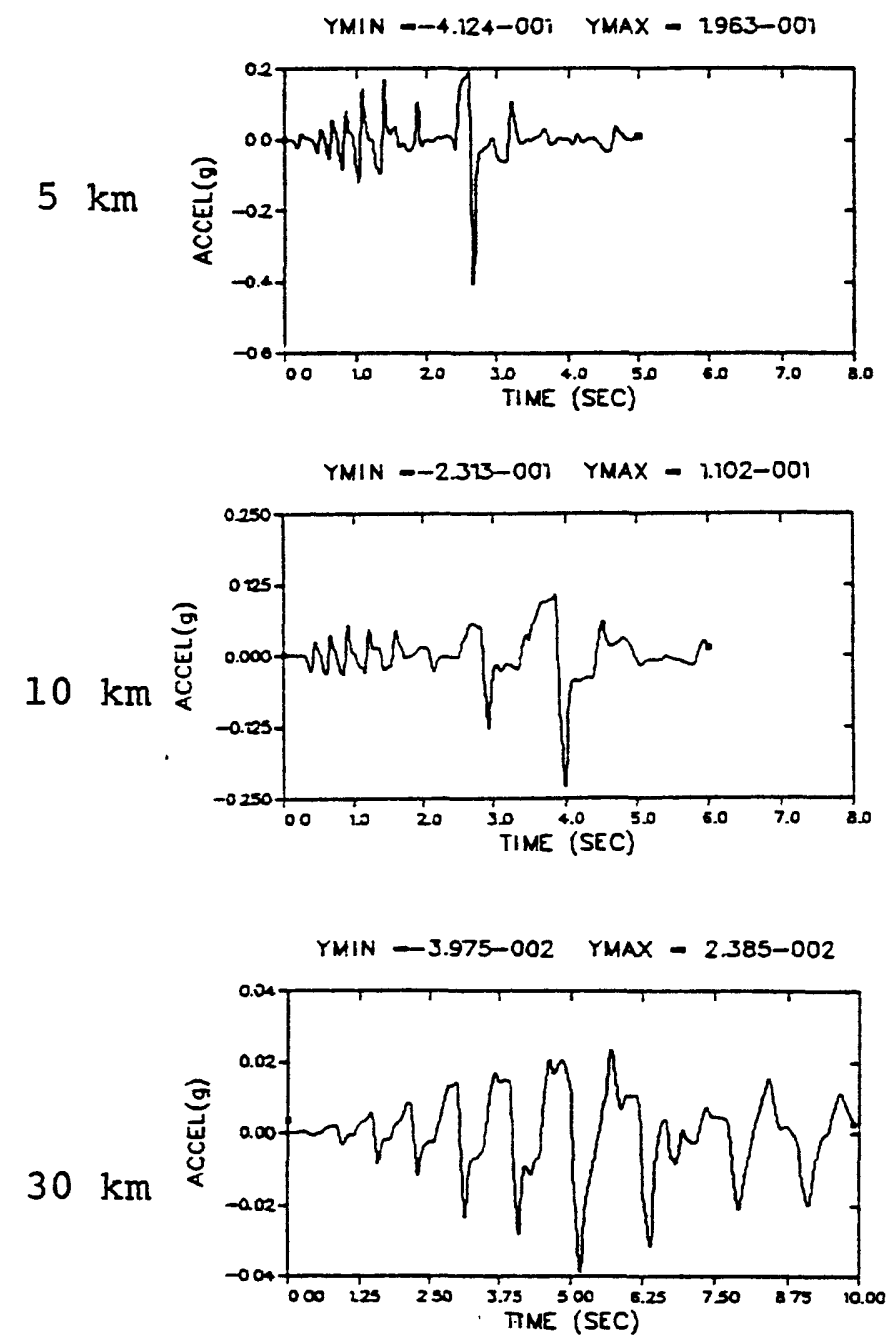

Figure 2.8. Simulated velocities and accelerations. 


\section{SANOIOHDS}

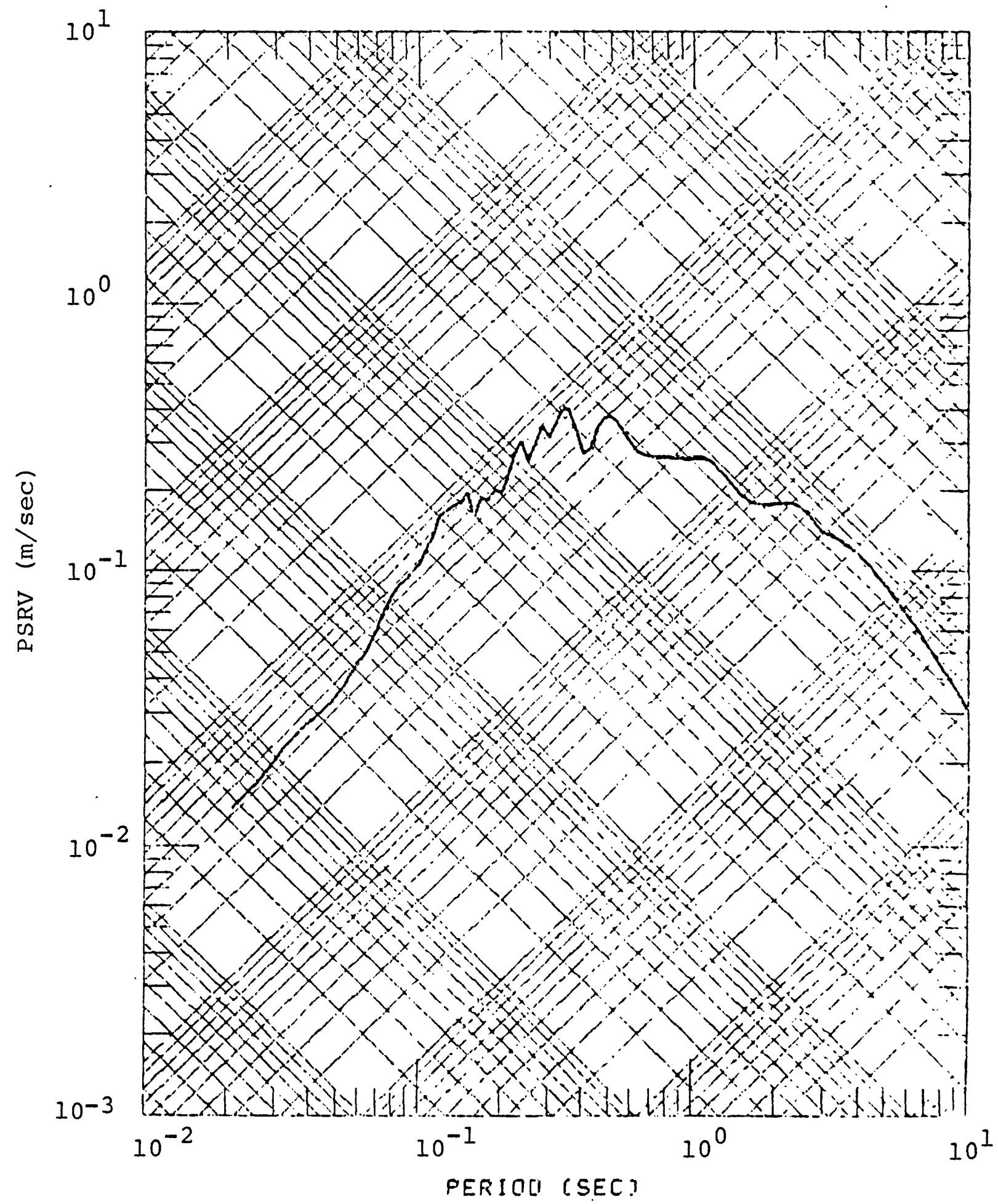

Figure 2.9. Pseudo-velocity spectra for 5 kilometer motion. 


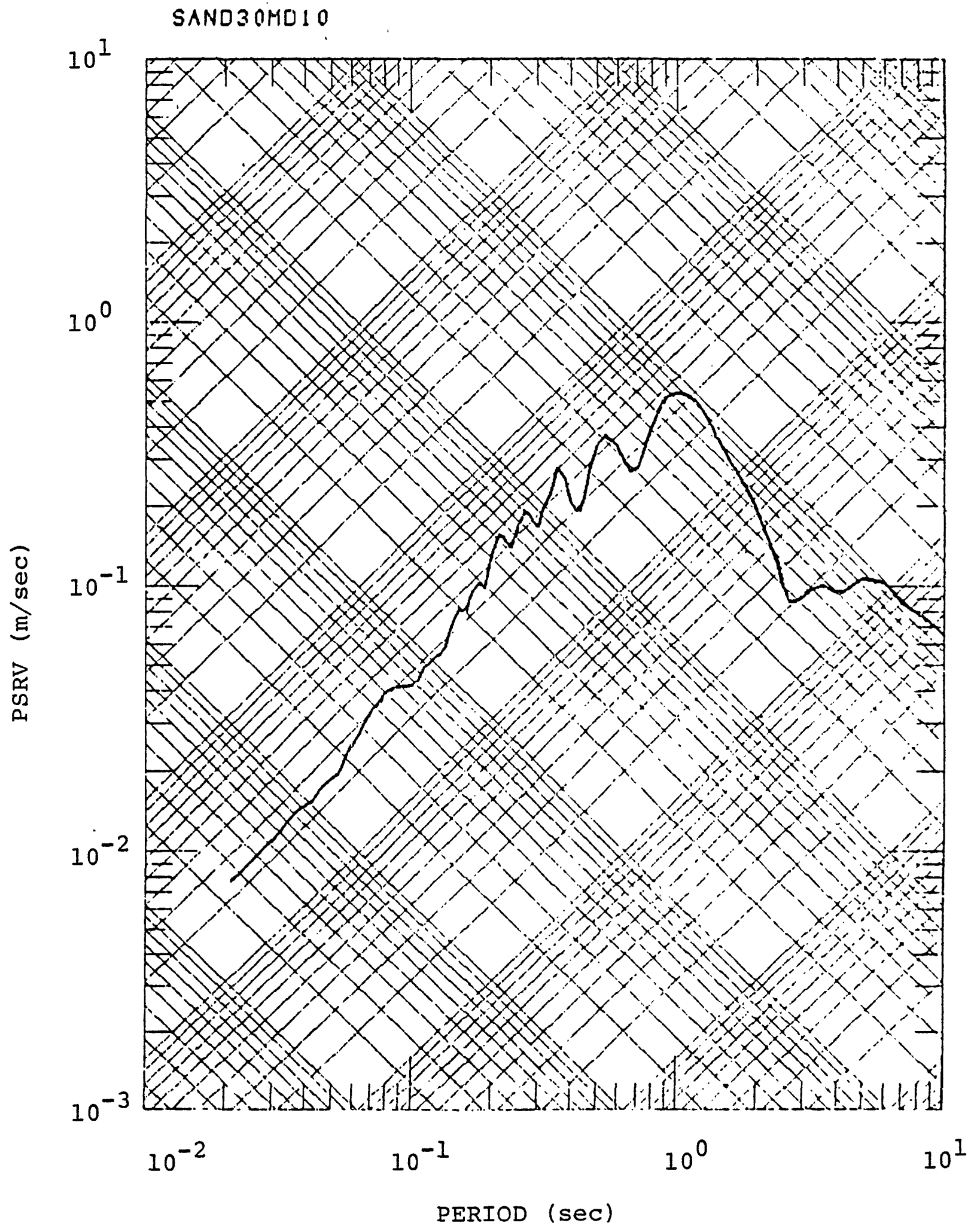

Figure 2.10. Pseudo-velocity spectra for 10 kilometer motion. 
SAMDZOMD3

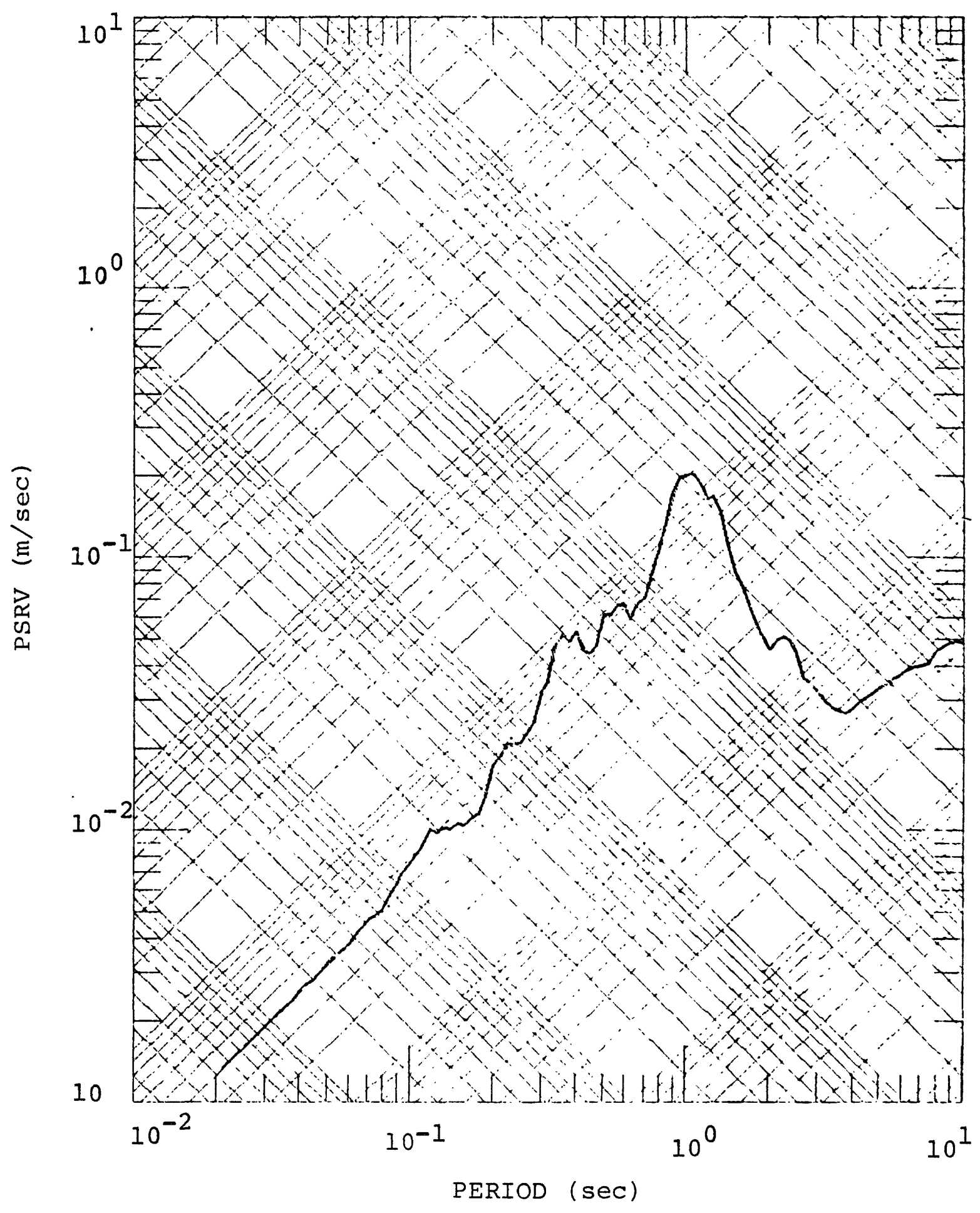

Figure 2.11. Pseudo-velocity spectra for 30 kilometer motion. 
operator preserves the integral of acceleration but not that of velocity. This causes an overestimation of the frequency content beyond $3 \mathrm{sec}$ periods at 10 and 30 kilometers but has almost no effect on the time window used at 5 kilometers. In cases where long periods are also of interest, more appropriate operators can be employed.

The source parameters were normalized to give reasonable fits to observed peak velocity and acceleration at 5 kilometers and 10 kilometers distance. The motion at 30 kilometers was not used to constrain the source model. When the peak values obtained at that distance were compared to recorded values (Figure 2.12), reasonable agreement was found with observations. One would expect the accelerations at such distances to be a sensitive function of the intrinsic attenuation assumed for the earth model. The values for shear $Q$ employed here are upper bounds of observed values and have only a small effect on the computed amplitudes. The decay of amplitude with distance is more strongly dependent on geometrical spreading and radiation pattern of the source. Even though shear Q's are probably the most poorly constrained earth parameters, the near-field motion may not be too sensitive to the values assumed.

\subsection{SUMMARY AND CONCLUSIONS}

A simple earthquake model consisting of discrete sources of concentrated stress release was used to synthesize the high frequency ground motion near a moderate-sized seismic event. A farfield radiation approximation was determined for each discrete source and was convolved with the appropriate Green's function for $\mathrm{SH}$-wave radiation in a layered earth structure. An important modification made to published far-field radiation from propagating shear cracks is the smoothing of stopping phases radiated from the fault edges.

The 1966 Parkfield, California, earthquake was used as a guide to specifying the source parameters. These parameters were chosen to obtain time histories, at distances of 5 and $10 \mathrm{~km}$ from the 


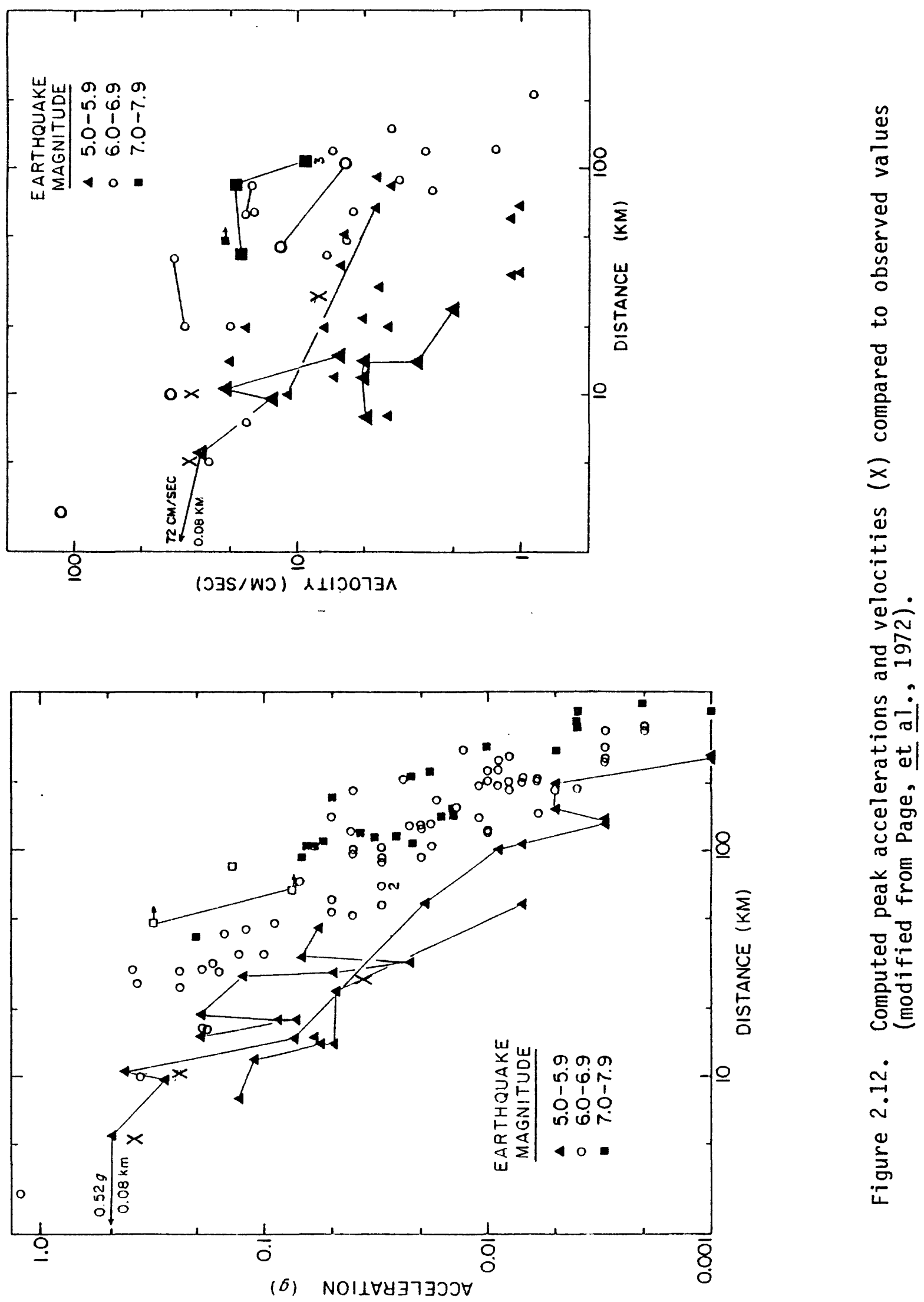


fault, with strong motion duration, peak acceleration, and peak velocity similar to those observed at Parkfield accelerograph stations number 5 and 8 . Using the same source model, ground motion was also synthesized for a receiver at $30 \mathrm{~km}$ from the fault, and the computed values of peak acceleration and velocity agree well with those observed from moderate-sized earthquakes at that distance.

The procedure employed here has several advantages over others which have been proposed. The use of a few discrete sources is considerably more practical economically than attempting a detailed integration over a large fault surface. The discrete source approach, when used with a layered earth response, can apparently produce complexity in the computed acceleration comparable to that observed in actual accelerograms, and this is achieved without recourse to purely statistical artifices. It has been suggested in the seismological literature that stress concentrations may be the most reasonable physical explanation for such complexity in observed acceleration.

Certain details of the procedure as applied in this study can be improved. It was assumed here that all sources were equally spaced over the fault and had the same source parameters. This is evidenced to some degree by the artificial regularities apparent in the computed time histories. Varying the size and location of the discrete events to more closely model the details of observed acceleration and velocity would substantially improve the scheme. The addition of P-SV radiation is also needed to improve results, and the required computational tools are currently being assembled. 


\section{SITE SOIL MODELS}

\subsection{BACKGROUND}

Because of increasing interest in the seismic safety and design of nuclear power plants, earth dams and other large civil systems, there has been considerable research activity during the past decade to more fully understand the behavior of soils under cyclic loading. Typical seismic disturbances produce highly irregular and complex loading of in situ soils, resulting from near-cyclic stress waves involving numerous reversals in the sign of the stress rate. When soils are subjected to such loading, they exhibit substantial noncoincidence of unloading-reloading paths (hysteresis), and strong dependence of the hysteresis on the history of deformation.

Early seismic designs were performed with highly simplified methods of analysis which treated the soil as linearly elastic material. For seismic disturbances of small amplitude, the use of elastic soil models is reasonable; however, such small amplitude disturbances are generally of no great concern to the seismic community. For seismic disturbances of sufficient magnitude to produce structural damage, the use of elastic models for soil response is unrealistic, since it implies stress levels in the soil that may be many times higher than the strength exhibited by the soil in the laboratory.

Today, the approach most commonly used by the earthquake engineering community to investigate seismic ground motion is the equivalent linear method (Idress and Seed, 1968). In this approach, the response of a soil-over-bedrock site is assumed to be governed by vertically traveling SH waves induced in the soil by the motion of the underlying bedrock. Soil behavior is modeled in an approximate manner by an equivalent linear shear model, whose properties are selected through an iterative procedure in accord with the average strain occurring at various depths in the soil. The equivalent linear method is attractive from a computational and 
an applications standpoint, and has provided adequate answers for many problems of interest. For the case of strong seismic disturbances, in which soil behavior is decidedly nonlinear, there is, however, some question about the accuracy of the equivalent linear method, and a number of investigations have recently been undertaken to examine this question, using truly nonlinear soil models. From these, it has been found that important differences arise between the response of a site predicted by truly nonlinear methods and that predicted by the equivalent linear method, especially for large amplitude motions (Joyner and Chen, 1975; Valera, et al, 1978; Taylor and Larkin, 1978). The nonlinear soil models that have been used in these nonlinear studies include the Ramberg-0sgood model (Streeter, Wylie and Rickart, 1974; Faccioli, Santayo and Leon, 1973), an elasto-plastic model (Richart, 1975), Iwan-type or sub-layer models (Joyner and Chen, 1975; Valera, et al, 1978, Taylor and Larkin, 1978; Bieber and Hovland, 1980), and the FLM model (Finn, Lee and Martin, 1975).

Each of the nonlinear models mentioned above has certain limitations in describing the general response of soils to the type of loading produced by a seismic disturbance. Some of the models are strictly one-dimensional, most do not allow for the effect of deformation history on hysteretic response, and none are capable of describing the phenomenon termed cyclic creep (ratcheting), which is commonly observed in soils subjected to cyclic loading about a prestressed state.

Recent advancements in computer technology have now made multi-dimensional nonlinear finite element analyses economically feasible for ground motion studies. As a result, there has been considerable activity during the past several years to develop multi-dimensional models for predicting the response of soils during cyclic loading.* Also, one such model has recently been used

*For an excellent summary of the latest advancements in the development of multi-dimensional soil models for describing cyclic behavior, see Pande and Zienkiewicz (1980). 
in conjunction with a large finite element code to analyze seismic-induced ground motion and corresponding soil-structure interaction (Isenberg, Vaughn and Sandler, 1978). It appears that the potential reduction in present design conservatism of nuclear power plants and other large civil systems that may be achievable through the use of such advanced methods of analysis could lead to very substantial savings in design and construction costs.

In the following sections, the two soil models utilized in the present study are described in detail. The first of these is the new endochronic model, which has just been recently introduced, and the second model is the equivalent linear model. Both models are applied to the cyclic simple shear data for drained sand reported by Cuellar, Bazant, Krizek and Silver (1977).

\subsection{THE ENDOCHRONIC SOIL MODEL}

In 1971, a new approach for modeling the rate-independent yet history-dependent response of inelastic materials was proposed (Valanis, 1971a; 1971b). This theory, called endochronic, was three-dimensional, and it differed from the previous theories of plasticity in that it did not require the concept of yield surface for its development. The theory was based on the hypothesis that the current state of stress in a material is a linear functional of the entire nistory of inelastic deformation, with the history defined with respect to a time scale, called intrinsic time, which is itself a property of the material at hand.

In the early efforts to apply the endochronic theory to real materials, attention was focussed mainly on metals (Valanis, 1974; Valanis and Wu, 1975; and Valanis, 1975). From this work, it became apparent that the theory was capable of predicting not only the gross features of inelastic metal response but also some observed features of metal plasticity that lay beyond the scope of existing plasticity theories. Furthermore, since the theory provided an 
approach for modeling materials having a vanishing elastic region and for which inelastic strain develops gradually from the onset of loading, its potential for modeling soils was quickly recognized (Bazant, 1974). In subsequent work, the ability of the endochronic approach to describe important features of soil behavior, including densification, hysteresis and liquefaction of sand under cyclic simple shear was demonstrated (Bazant and Krizek, 1976; Cuellar, Bazant, Krizek and Silver, 1977). The work of Bazant and his co-workers was, nevertheless, largely limited to simple shear, and there was a need to explore the potential of the theory under more complex states of deformation.

The original endochronic theory, which Bazant adopted and modified to encompass several effects characteristic of soils was, however, unable to predict closed hysteresis loops for small unload-reload processes under one-dimensional conditions. For such processes, the theory predicted a slope at the reloading point that was smaller than the unloading slope at the same point. This feature of the theory did not appear to be in agreement with the observed behavior of most materials, including soils, although it must be admitted that there are very few data available on the response of materials to such processes to either verify or invalidate this feature of the theory. Nonetheless, it seemed reasonable to assume that most materials exhibit elastic behavior at, and in the small neighborhood of, the point of unloading, and this was not the case with the original endochronic theory. Furthermore, some suspicions were raised that the small unloadreload feature of the theory could lead to numerical difficulties if the model was used in conjunction with computer codes to analyze wave propagation problems (Sandler, 1978), although this was never demonstrated numerically in a well-defined wave propagation problem.

In 1978, $\mathrm{s}^{3}$ developed a general endochronic model for soils, which was intended for use in conjunction with advanced finite element codes for analyzing seismic ground motion and soil-structure 
interaction problems (Valanis and Read, 1978; Read and Valanis, 1979). From this investigation, a soil model was developed which provided several improvements over the earlier theory but still suffered from the lack of hysteresis loop closure for small unload-reload processes, although some degree of improvement in this regard was obtained.

In a subsequent investigation, $\mathrm{S}^{3}$ reexamined the problem of hysteretic loop closure, and from this effort, a new endochronic soil model was formulated (Valanis, 1979; Valanis and Read, 1979; Valanis and Read, 1980). The new theory is not only free of the undesirable small unload-reload response feature, but provides greater modeling capability with remarkably few material parameters. It also has the capability to describe most of the features of soil behavior important for seismic loading, including densification, dilatancy, strain hardening/softening, hysteresis and cyclic creep (ratcheting).

In the present study, the new endochronic soil model is used to describe the behavior of the soil (sand) at the site under consideration. The simple shear version of the model used for this purpose is described below and its application to cyclic simple shear data for drained sand is illustrated.

\subsubsection{Endochronic Model for Simple Shear of Sand}

A detailed development and discussion of the basic equations that describe the new endochronic soil theory has been recently given (Valanis and Read, 1979, 1980), and will not be repeated here. Instead, we go directly to the equations that describe simple shear behavior, which are listed below:

$$
\begin{aligned}
s & =H(z) \int_{0}^{z} \rho\left(z-z^{\prime}\right) \frac{d \theta}{d z^{\prime}} d z^{\prime} \\
d s & =G\left(d_{\gamma}-2 d \theta\right) \\
d z & =|d \theta|
\end{aligned}
$$


Here, $s$ represents the shear stress, $\gamma$ is the engineering shear strain, $2 \theta$ denotes the plastic strain component, and $z$ is the intrinsic time. Furthermore, $G$ represents the elastic shear modulus, $H(z)$ is a hardening/softening function, and $\rho$ denotes a weakly singular kernel function having the property $\rho(0)=\propto$. Eq. 3-1 is the inelastic constitutive relation that describes the shear stress as a linear functional of the plastic strain history, while Eq. 3-2 is simply an incremental form of Hooke's law. Eq. 3-3 defines the monotonically increasing intrinsic time in terms of the increment of plastic strain. The above system of equations contains two functions, $H(z)$ and $\rho\left(z-z^{\prime}\right)$, whose forms must be specified for a given material.

In the case of loose sand, the following forms of $H$ and $\rho$ appear to provide an excellent description of the cyclic simple shear over many cycles of deformation:

$$
\begin{aligned}
& \rho\left(z-z^{\prime}\right)=\frac{e^{-k\left(z-z^{\prime}\right)}}{\sqrt{z-z^{\prime}}} \\
& H(z)=H_{0}+\left(H_{\infty}-H_{0}\right)\left(1-e^{-\eta z}\right)
\end{aligned}
$$

where $k, H_{0}, H_{\infty}$ and $n$ are material-dependent constants.

Upon substituting Eqs. 3-4 and 3-5 into Eq. 3-1, and considering a deformation history involving numerous reversals in the shear strain rate, it can be shown (Valanis and Read, 1979) that Eq. 3-1 can be recast in the following form:

$$
\begin{aligned}
s & =H(z)\left\{F(z)-2\left[F\left(z-z_{1}\right)-F\left(z-z_{2}\right)\right.\right. \\
& \left.\left.+\ldots(-1)^{n+1} F\left(z-z_{n}\right)\right]\right\}
\end{aligned}
$$

where $z_{1}, z_{2}, \ldots z_{n}$ denote the values of the intrinsic time $z$ at which reversals in the sign of the shear strain rate have occurred, and $F(z)$ is defined as: 


$$
F(z)=\operatorname{Erf}(\sqrt{k z})
$$

where Erf ( ) denotes the error function. Equations 3-2, 3-3 and $3-6$, with $H(z)$ and $F(z)$ defined by Eqs. $3-5$ and $3-7$, constitute a set of simultaneous equations that can be solved by numerical methods to give the shear stress $s$ for a prescribed shear strain history.

It is worthwhile to point out a feature of the simple shear model described above that can greatly increase the computing efficiency of a numerical scheme for these equations. Note that the right hand side of Eq. 3-6 contains an increasing number of terms as the number of cycles, and hence the number of reversals in the sign of the shear strain rate, increases. In general, there will be $2 n+1$ terms after $n$ cycles of deformation. Also, the values of the $2 n$ quantities $z_{1}, z_{2}, \ldots z_{2 n}$ must be retained in computer memory. This implies that when there are many cycles of deformation, the model could become computationally unattractive.

Some important advantages occur from a computing standpoint, however, if the function $F(z)$ has a form which saturates to a limiting value as $z$ increases, which is the case for the $F(z)$ given in Eq. 3-7.* When saturation, or near saturation, of a term occurs there is, of course, no further need to calculate the term, since for all future deformation the intrinsic time continues to increase and the term remains at its limiting value. Similar comments apply also to the general term $F\left(z-z_{j}\right)$. Secondly, when a term $F\left(z-z_{j}\right)$ saturates, it no longer becomes necessary to retain in computer memory the value of $z_{i}$, or for that matter, any of the $z_{j}$ which are less than $z_{i}$; under some circumstances, this can significantly reduce the computer storage requirement.

*When the function $F(z)$ has a form that saturates to a limiting value as $z$ increases, we can say that the corresponding endochronic model exhibits "fading memory." 
Using the simplifications noted above, a numerical scheme was formulated to calculate the shear stress from a prescribed strain history. Further developments were required, however, to obtain a numerical scheme adequate for treating the complex strain histories encountered in the numerical wave propagation simulations. Those developments will be described in Section 4.3.1. The governing system of equations was solved by an iterative approach based on Newton's method, using the computer subroutine described in Appendix C.

\subsubsection{Application of Model to Cyclic Data for Sand}

Cuellar, Bazant, Krizek and Silver (1977) have presented considerable data on the response of drained sand to numerous cycles of simple shear. Their studies were performed with a simple shear device that was a modified version of the type initially designed by the Norwegian Geotechnical Institute. The cyclic tests were performed on cylindrical samples of sand, which were enclosed in a wire-reinforced membrane. The end surfaces of the cylindrical samples were subjected to cyclic relative displacements normal to the axis of the samples, while a fixed distance was maintained between these surfaces. The frequency of the cyclic relative displacement was $1 \mathrm{~Hz}$. Moreover, each test was conducted with a dead load applied to the specimen along the direction of the cylinder axis.

Although the experimental device described above is commoniy referred to as a "simple shear" device, it is well known that the states of stress that it produces in a soil specimen are not strictly simple shear. In order to have simple shear, uniform shear stresses would have to be applied over the entire surface of the sample, including the curved lateral surface of the cylindrical specimen; this is not, of course, the boundary condition applied by the wire-reinforced flexible membrane. Furthermore, although such 
tests are typically performed under a constant vertical load, one finds that the pressure in the soil does not remain constant during a test, but increases due to the build-up of the lateral stresses from hardening.

A detailed analysis of the response of sand in the type of cyclic simple shear device described above has recently been performed with a dynamic finite element code (Bazant, Krizek and Shieh, 1979). The results from this study reveal that the calculated cyclic response of sand in simple shear device is similar to that calculated under the assumption of simple shear. Consequently, this result provides some rationale for using data from such devices to evaluate material parameters in simple shear models.

On this basis, the endochronic simple shear model described earlier was applied to the cyclic simple shear data for drained sand given by Cuellar, Bazant, Krizek and Silver (1977). The particular data considered corresponded to an initial relative density of 45 percent, an applied vertical stress of $192 \mathrm{kPa}$, and a peak shear strain of 0.3 percent.

The initial state from which the intrinsic time $z$ is measured was taken to be the state prior to the initiation of shearing. A more precise treatment would take the compressed state of the material prior to the application of the vertical load as the initial state. However, no information was available on the deformation that occurred during vertical load application, and consequently the corresponding increment in intrinsic time could not be determined.

Using the numerical subroutine listed in Appendix $C$, the endochronic simple shear model was fit to the cyclic simple shear data by an iterative procedure. This was achieved by using the actual strain history measured during the experiments to drive the calculations. Moreover, a uniform strain increment of $\Delta \gamma=0.01$ percent was used in the calculations. Excellent agreement between 
the data and the calculations was obtained over 300 cycles of response with the following values of the simple shear model parameters:

$$
\begin{array}{rlrl}
\mathrm{G} & =39.5 \mathrm{MPa} & H_{\infty}=74.7 \mathrm{kPa} \\
H_{0} & =28.73 \mathrm{kPa} & n=61.2 \\
\mathrm{k} & =1500 & n &
\end{array}
$$

The calculated stress-strain paths for cycles $1,2,10$ and 300, based on the values of the parameters listed above, are shown in Figure 3.1. Also shown on these figures are the corresponding experimental data from Cuellar, Bazant, Krizek and Silver (1977), which is denoted by the open circles; the dashed curves in these figures are simply smooth curves drawn through the data. The close agreement between the calculated and observed response of the sand over 300 cycles of deformation illustrates the powerful capability of the new endochronic theory to describe the complex hysteretic behavior of soils over many cycles of deformation. Note also, that the model requires only five parameters to accomplish this.

Another interesting feature of the endochronic soil model is its ability to describe cyclic creep, or ratcheting, a commoniy observed characteristic of soils subjected to cyclic loading about a prestressed state. To illustrate the ability of the model to describe this phenomenon, the simple shear model described above was cycled between stress limits of $28.73 \mathrm{kPa}$ and $2.39 \mathrm{kPa}$ for 30 cycles, after initial loading to the upper stress limit. The calculated responses of the model for cycles 1 to 3,10 and 30 is shown in Figure 3.2, which illustrates the growth in strain that occurs with increasing cycles of deformation. Note that the predicted response approaches elastic behavior with increasing cycles of deformation due to the hardening present in the model.

\subsection{THE EQUIVALENT LINEAR MODEL}

The endochronic model described in the previous section accurately simulates the stress-strain response of geologic 

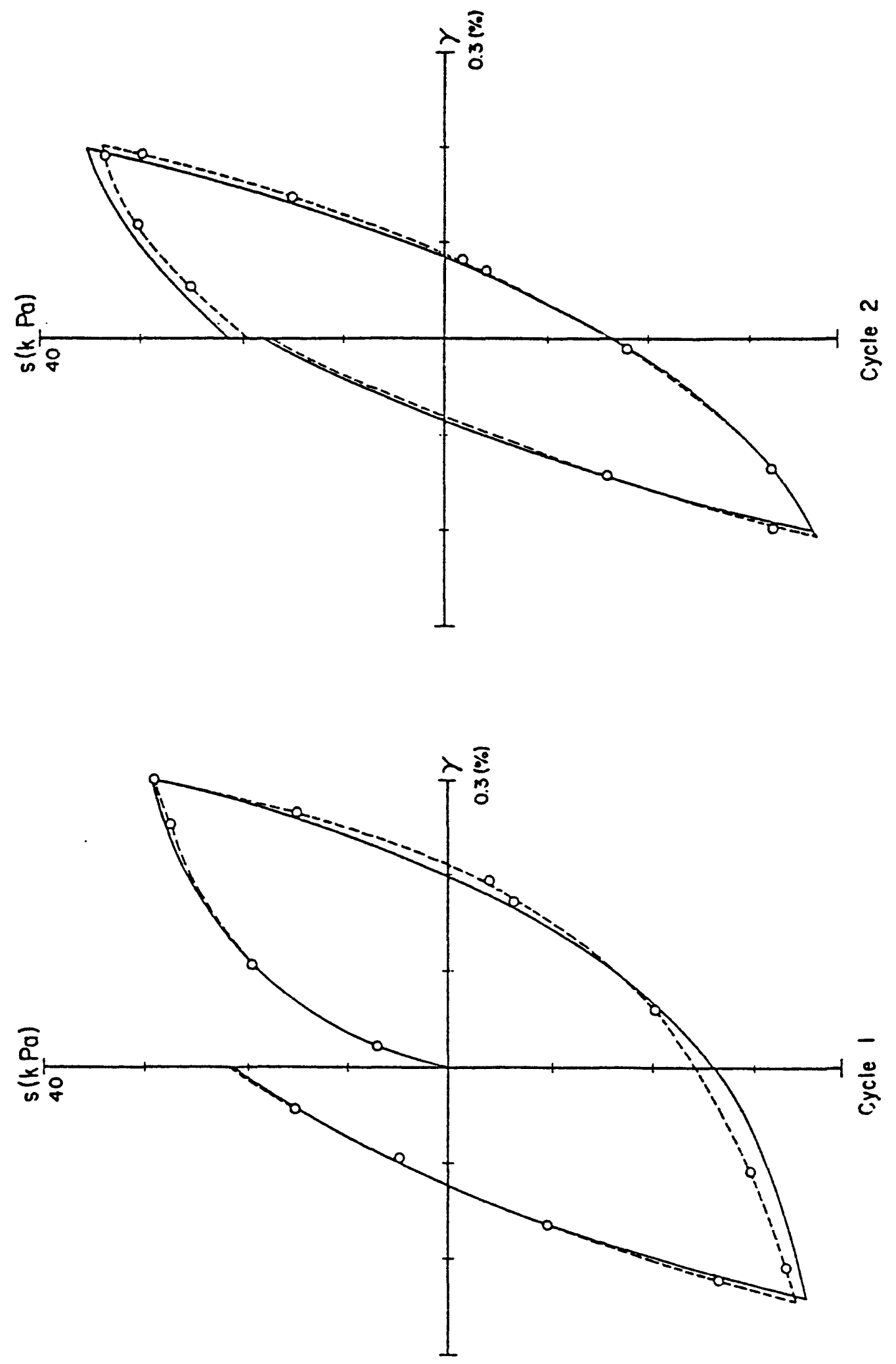

4

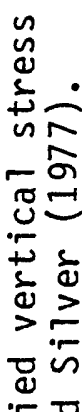

묻

응

$=\stackrel{N}{N}$

这

气

$\frac{\pi}{\pi} \stackrel{\pi}{\pi}$

$\frac{\infty}{n}$

ज $\frac{2}{\sigma}$

풍

ปै

웅

다

ปิ

40

40

ब

동

음

ฝิ๊

$\ddot{-}$

崩 


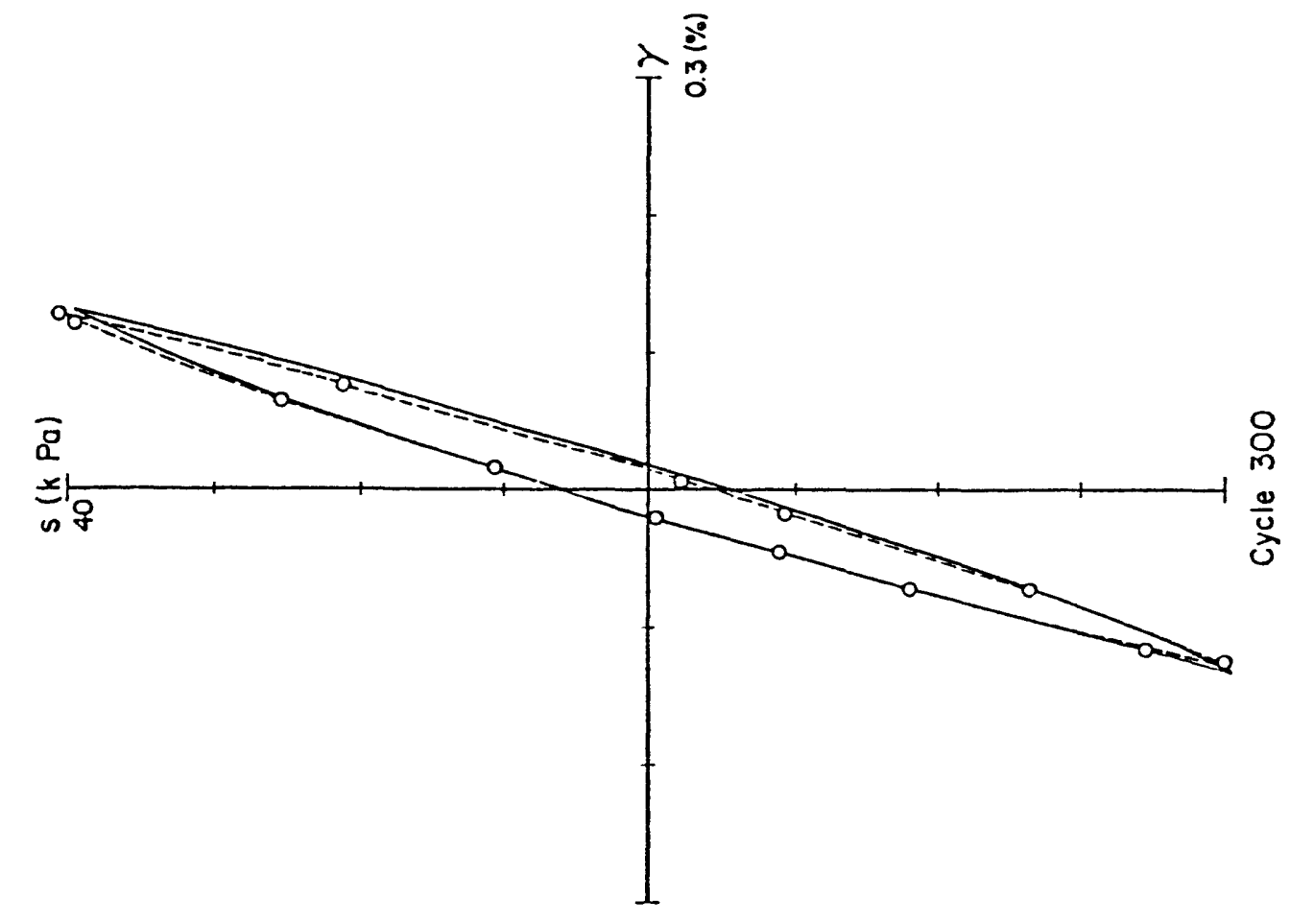

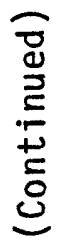

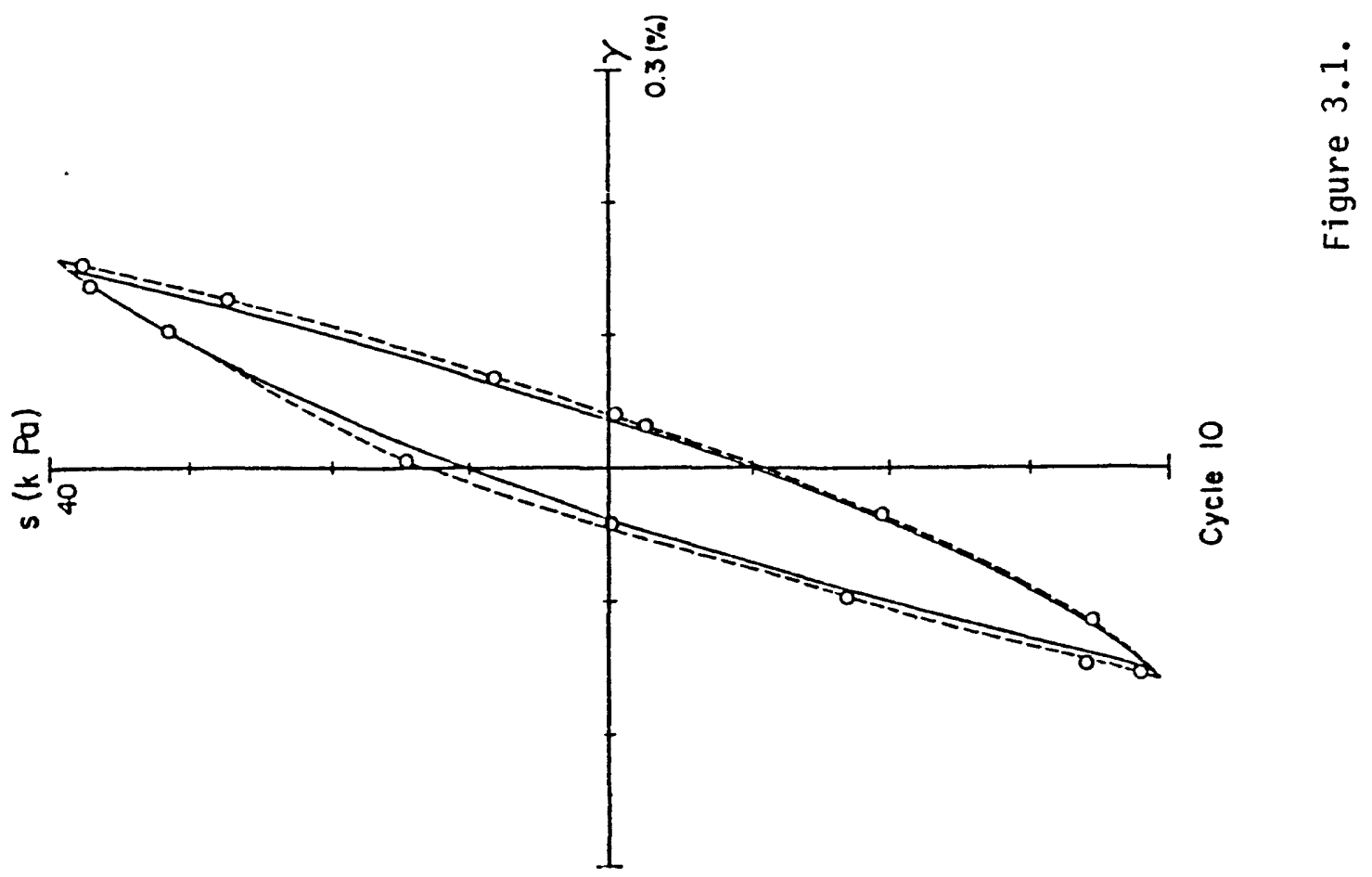



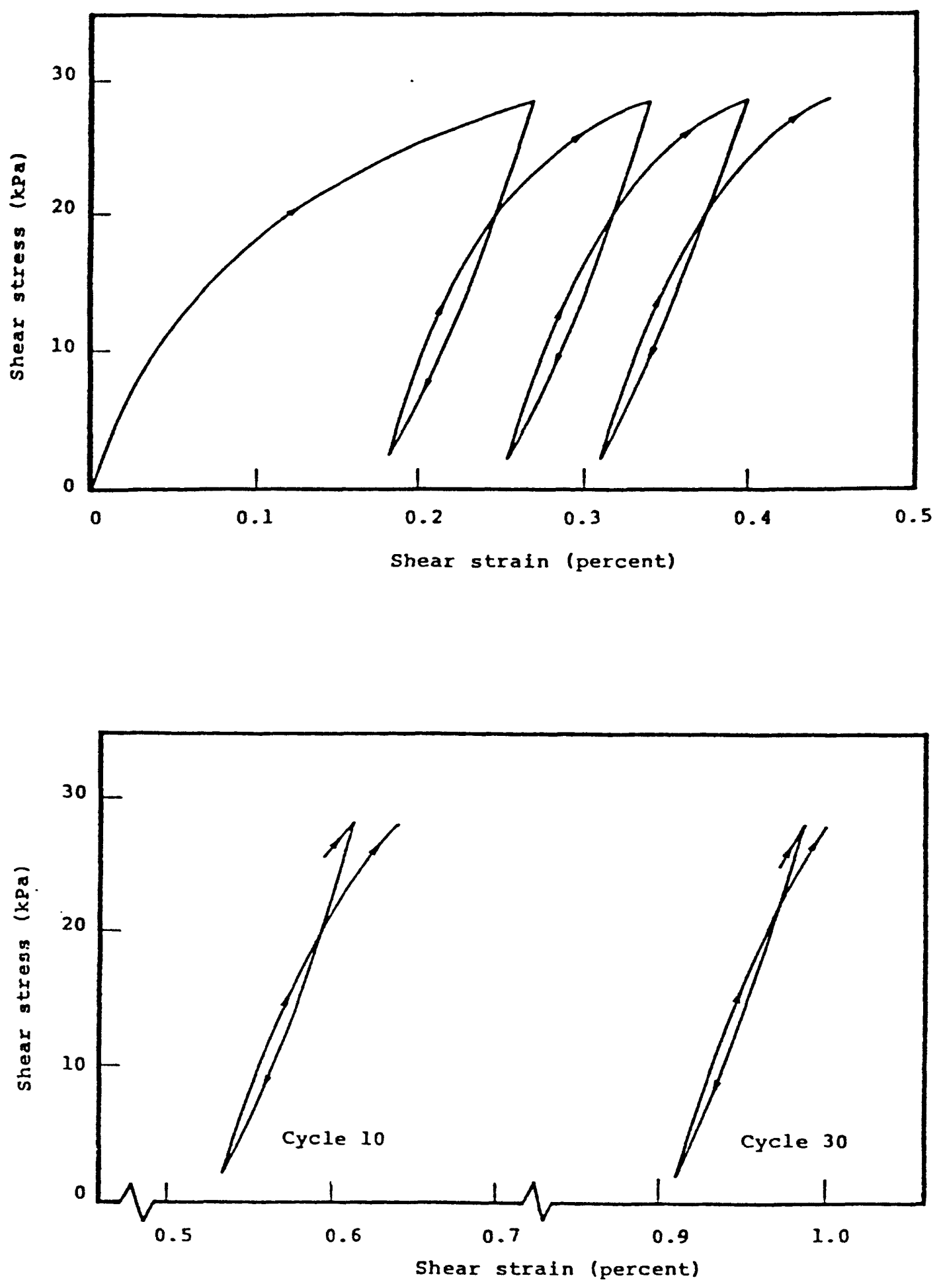

Figure 3.2. Response of endochronic shear model to asymmetric cyclic deformation, illustrating the phenomenon of cyclic creep (ratcheting). 
materials experiencing cyclic deformation. Furthermore, and in contrast to conventional eauivalent linear models, the endochronic model is a three-dimensional theory. On the other hand, the model can only be used in computer codes which permit nonlinear material response and solve boundary value problems by direct time integration. Thus, the potentially greater accuracy of the endochronic model relative to conventional, inherently one-dimensional equivalent linear models must be weighed against the increased cost associated with its utilization.

To assess differences in response predictions between the endochronic model and more conventional theories of soils, an equivalent linear model was derived for the dry sand described earlier and incorporated into the SHAKE code. In the equivalent linear approach, the soil profile is divided into a set of horizontal layers and strain dependent material properties for the constituent soils specified. In each layer, constant, uniform material properties are determined iteratively, the iteration process terminating when the strains computed in each layer match the strains used to evaluate layer material properties.

The SHAKE code models each layer using linear viscoelastic theory wherein the complex-valued shear modulus takes the frequency-insensitive form

$$
G^{\star}=G(1+2 i B) \text {. }
$$

Here $B$ is a measure of the soil critical dampling ratio, $G$ is the elastic shear modulus, and $i=(-1)^{1 / 2}$.

Equivalence between the equivalent linear model and the endochronic theory was established using the method described in Joyner and Chen (1975). In that method the viscoelastic model was exercised around the shear strain path

$$
r=\gamma_{\max } \cos \omega t
$$

$G$ and $B$ were determined such that the maximum shear stress, and the energy dissipation per unit volume per cycle, $W$, 
equalled that of the endochronic model when it was cycled between the strain limits $-\gamma_{\max } \leq \gamma \leq \gamma_{\max }$. For the endochronic theory a typical first cycle hystersis loop is shown in figure 3.3. As can be seen in the figure, the loop is not closed, thus $s_{\max }$ was taken to be the average of the stress at the beginning and end of the cycle, i.e., $\left(s_{1}+s_{2}\right) / 2$. The energy dissipation $W$ was computed as the area inside the loop.

Energy dissipation per cycle for the viscoelastic model is

$$
W=\int_{0}^{2 \pi / \omega} s \dot{\gamma} d t=\pi r_{\max } s_{\max } \sin \delta
$$

where

$$
\sin \delta=2 B /\left(1+4 B^{2}\right)^{1 / 2} \text {. }
$$

For the harmonic strain history, Ea. (3-10), we have

$$
S=\gamma_{\max } G\left(1+4 B^{2}\right)^{1 / 2} \cos (\omega t+\delta)
$$

Thus, from Ea. (3-13)

$$
S_{\max }=r_{\max } G\left(1+4 B^{2}\right) 1 / 2 .
$$

From Eas. (3-11) to (3-14) it follows that

$$
\begin{aligned}
& B=\frac{W}{2}\left(\pi^{2} r_{\text {max }}^{2} s_{\text {max }}^{2}-W^{2}\right)^{-1 / 2} \\
& G=\left(\frac{s^{2} \text { max }}{r_{\text {max }}^{2}}-\frac{W^{2}}{\pi^{2} r_{\text {max }}^{4}}\right)^{1 / 2}
\end{aligned}
$$

To account for strain magnitude sensitivity, $G$ and $B$ were computed for five values of $\gamma_{\max }$; then logarithmic interpolation 


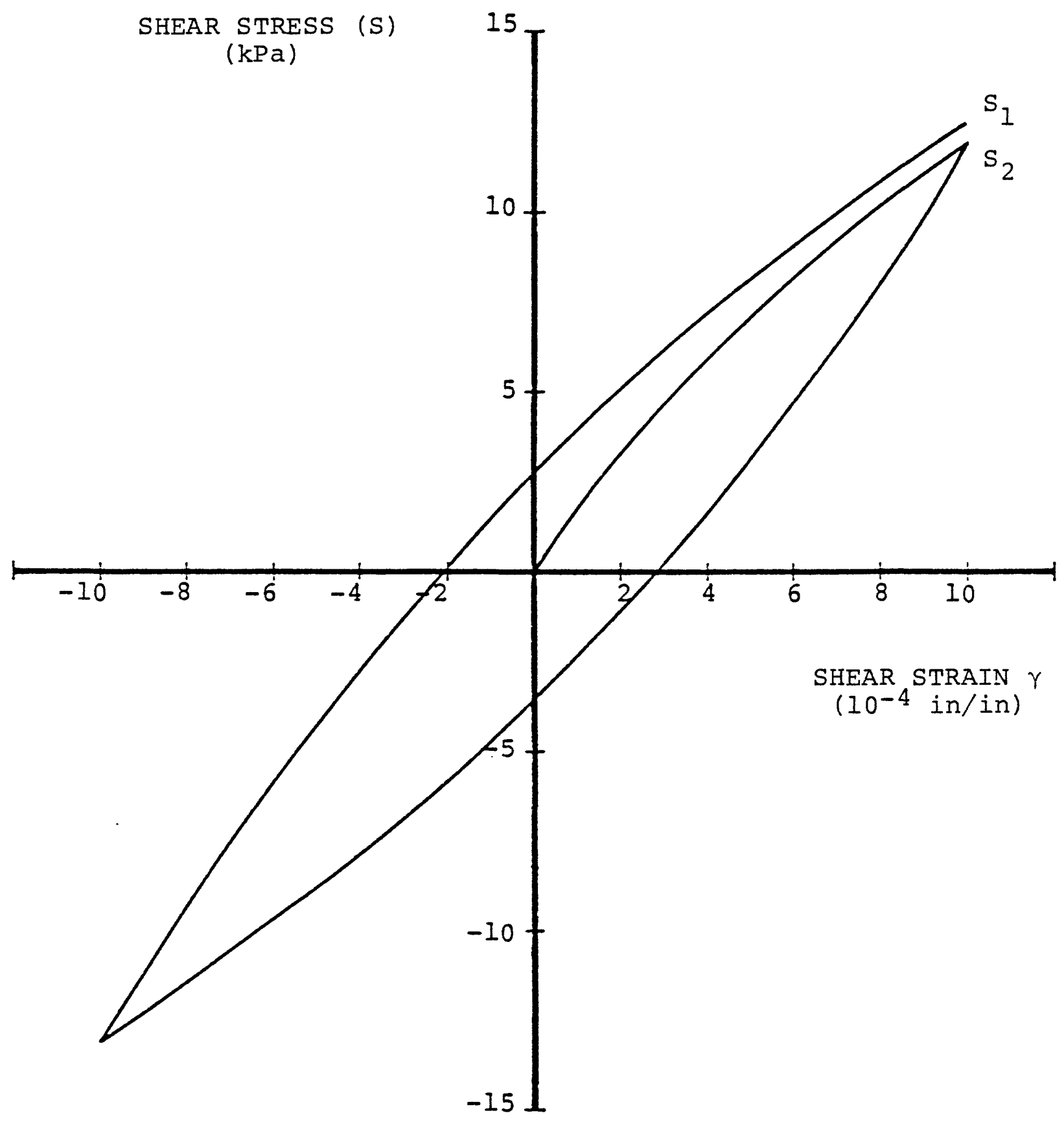

Figure 3.3. First cycle stress-strain response for crystal silica No. 20 sand loaded in shear between fixed strain limits using endochronic model. 
was used in the SHAKE code to compute $G$ and $\beta$ at intermediate values of strain. In the present program the material modeled was the crystal silica number 20 sand, discussed by Cuellar, et al (1977), and the computed values of $G$ and $\beta$, along with values of $\gamma_{\max }$, $S_{\max }$ and $W$ are given in Table 3.1. All material properties were based on experimental data obtained from simple shear tests where the confining pressure was $114.9 \mathrm{kPa}$. 
TABLE 3.1

STRAIN DEPENDENT SHEAR MODULI G AND CRITICAL DAMPING RATIOS

B USED IN SHAKE CALCULATIONS.

CONFINING PRESSURE WAS $114.9 \mathrm{kPA}$.

\begin{tabular}{ccccccc}
$\gamma \max$ & $S_{\max }(\mathrm{kPa})$ & \multicolumn{2}{c}{$W\left(\mathrm{~J} / \mathrm{m}^{3}\right)$} & $G(\mathrm{MPa})$ & $\beta$ \\
$10^{-5}$ & 0.196 & 1.446 & $\times$ & $10^{-5}$ & 19.56 & 0.001763 \\
$10^{-4}$ & 1.815 & 1.182 & $\times$ & $10^{-2}$ & 18.14 & 0.01037 \\
$10^{-3}$ & 12.293 & 4.3 & $\times$ & $10^{0}$ & 12.21 & 0.056066 \\
$10^{-2}$ & 41.489 & 5.79 & $\times$ & $10^{2}$ & 3.716 & 0.24776 \\
$10^{-1}$ & 69.042 & 1.38 & $\times$ & $10^{4}$ & 0.532 & 0.41406
\end{tabular}




\section{SOIL SITE GROUND MOTION}

\subsection{INTRODUCTION}

In Section 2, a deterministic approach was employed to construct synthetic accelerograms at a range of distances from a strike-slip fault. The synthetic ground motion was evaluated for sites on rock (shear velocity equal to $980 \mathrm{~m} / \mathrm{sec}$ ). The method, though limited to SH waves, incorporated source finiteness, elastic response of the crust, and anelastic attenuation, in a fairly rigorous manner.

For soils, however, the linear viscoelastic treatment used for rock sites would be inappropriate. Section 3 described a new nonlinear constitutive model appropriate for soils, the endochronic model, and illustrated its capability to describe cyclic simple shear data for dry sand. Then, an equivalent linear representation was derived for the same dry sand simple shear data.

In this section, we extend the modeling study of ground motion to sites on cohesionless soil. Our primary objective is to examine the predictive capabilities of the deterministic modeling approach to site-dependent ground motion estimation, taking account of the nonlinear response of soil deposits. The capabilities of the approach are evaluated through comparisons with ground motion data and equivalent linear analyses.

The sites studied are assumed to overlie the earth structure employed in Section 2 so that the synthetic accelerograms computed in Section 2 provide the upgoing wave motion at the base of the soit deposit. The response of the soil deposit is described by the endochronic model of dry sand developed in Section 3.2.2. The transient response of the soil deposit is then obtained by a finite element method, assuming simple shear deformation (vertically propagating $\mathrm{SH}$ waves) in the soil column. We repeat the analysis of the soil column response using the SHAKE code with the equivalent linear representation derived in Section 3.3. Section 4.2 describes the set of calculations which was performed for the study, Section 4.3 describes the numerical methods employed, and Section 4.4 
presents and discusses the numerical results. In the discussion of results, detailed consideration is given to (1) the performance of the endochronic constitutive model in describing complex stress histories, (2) the sensitivity of the nonlinear analysis to details of the soil profile discretization, (3) comparison of the nonlinear results with trends in earthquake data, and (4) comparison of the nonlinear solutions with those obtained using the equivalent linear model.

\subsection{DESCRIPTION OF THE NONLINEAR NUMER ICAL STUDIES}

Table 4.1 summarizes the eight problems treated in this study. Three different soil profiles, denoted A, B, and $C$, were considered. We also considered three different depths to bedrock -10, 30, and 100 meters. Finally, four different synthetic accelerograms were assumed for the bedrock input motion: the bedrock accelerograms at 5,10 , and $30 \mathrm{~km}$ distance, respectively, from Section 2, as well as the synthetic accelerogram at $5 \mathrm{~km}$ distance, rescaled to give a peak acceleration of $1 \mathrm{~g}$. Table 4.1 also summarizes the peak acceleration and velocity values obtained at the ground surface in each calculation. No equivalent linear analysis was performed for soil profiles $B$ and $C$, since these profiles were introduced to check the sensitivity of the nonlinear calculations to small variations in the soil profile.

\subsubsection{Soil Profiles Employed}

Three soil profiles, denoted $A, B$, and $C$, were examined in this study. Each approximates the properties of the drained sand to which the endochronic constitutive model was fit in Section 3.2.2.*

\footnotetext{
* The values of the endochronic soil model parameters for dry sand $(G, k$, and $n$ ) given in Section 3.2.2., Equation 3-8, differ from those reported in Valanis and Read (1980), due to an error in the earlier report. The numerical calculations described here were completed prior to discovery of this error, and employed the numerical values $G=19.75 \mathrm{mPa}, k=750, n=30.6$. Since the objectives of this study do not rely on replicating the response of a particular soil sample, this discrepancy is not of concern. Nevertheless, one of the nonlinear calculations was subsequently repeated using numerical values consistent with the experimental data fit in Section 3.2.2, with the result that peak values of acceleration and velocity obtained using the two sets of soil parameters differed by only a few percent.
} 


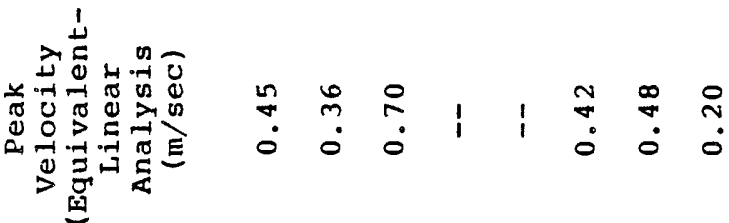

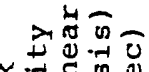

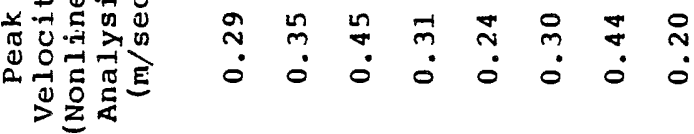

$$
\begin{aligned}
& \stackrel{5}{5}
\end{aligned}
$$

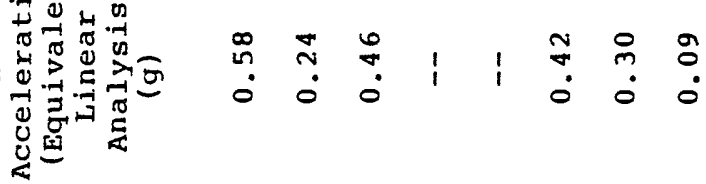

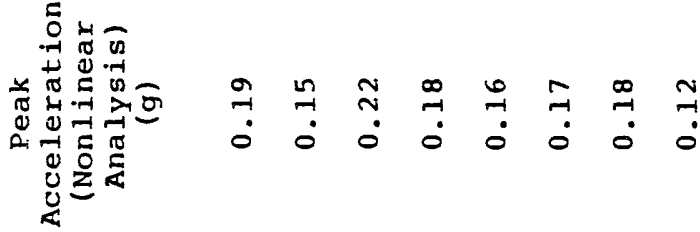

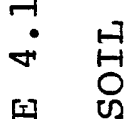

$$
\begin{aligned}
& \text { 量 鼻 }
\end{aligned}
$$

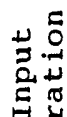

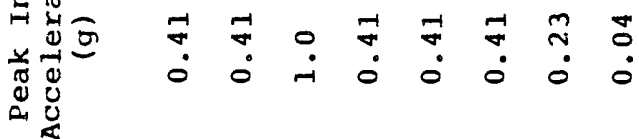

$$
\begin{aligned}
& \text { 형 }
\end{aligned}
$$

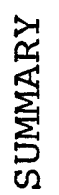

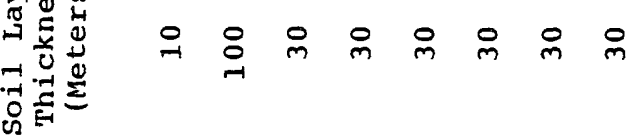

$$
\begin{aligned}
& \sum_{0}^{\infty} 0 \\
& \text { 员运踏 } \\
& \text { 至 } \\
& <<<\infty<<<
\end{aligned}
$$

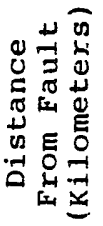

$$
\begin{aligned}
& n \text { in } n \text { un in in } 0 \\
& \text { 竞苍 } \\
& H \sim m-\infty \infty
\end{aligned}
$$


The depth dependences of the shear modulus, $G$, and the shear strength, $H_{\infty}$, are discussed in Appendix $B$, and the three soil profiles differ in the way in which such depth dependences are approximated. In Profile $A$, the depth dependences are averaged over layers which vary in thickness, with a minimum thickness of 2 meters in the upper part of the profile; the resulting values of $G, H_{\infty}$, and the shear velocity, $V_{S}$, are shown in Figure 4.1. In Profile $B$, shown in Figure 4.2., the very low values of $G$ and $H_{\infty}$ present in the uppermost layer of Profile $A$ have been increased, and the remainder of Profile $B$ is a smoothed version of Profile $A$. Profile $C$ is assigned uniform values of $G$ and $H_{\infty}$, as shown in Figure 4.2.

In each case, the soil deposit is assumed to have a uniform density of $1462 \mathrm{~kg} / \mathrm{m}^{3}$, and to overlie a base-rock with density $2100 \mathrm{~kg} / \mathrm{m}^{3}$, and and to have a shear velocity $980 \mathrm{~m} / \mathrm{sec}$. The endochronic simple shear parameters $k$ and $n$, and the ratio $H_{0} / H_{\infty}$, are assumed to be independent of depth.

\subsubsection{The Input Ground Motion}

As already noted, the base-rock input transients for the soil site analyses were developed from the synthetic accelerograms generated for rock sites at distances of 5, 10, and $30 \mathrm{kilometers} \mathrm{from}$ a simple strike-slip fault model. The method of driving the soil column accounts for the fact that the base-rock motion is modified by the presence of the soil. The upgoing wave motion computed for rock sites is imposed at the base of the soil by means of superposed body forces, while downgoing waves produced by the presence of the soil and free surface are transmitted back into the base-rock.

An alternate approach would have been to drive the soil calculations using actual accelerograms recorded on bedrock at various source-receiver distances. An advantage of that approach would have been to eliminate uncertainties associated with our simplistic source model and our neglect of the P-SV wave contribution to ground motion. However, the latter might also be a 


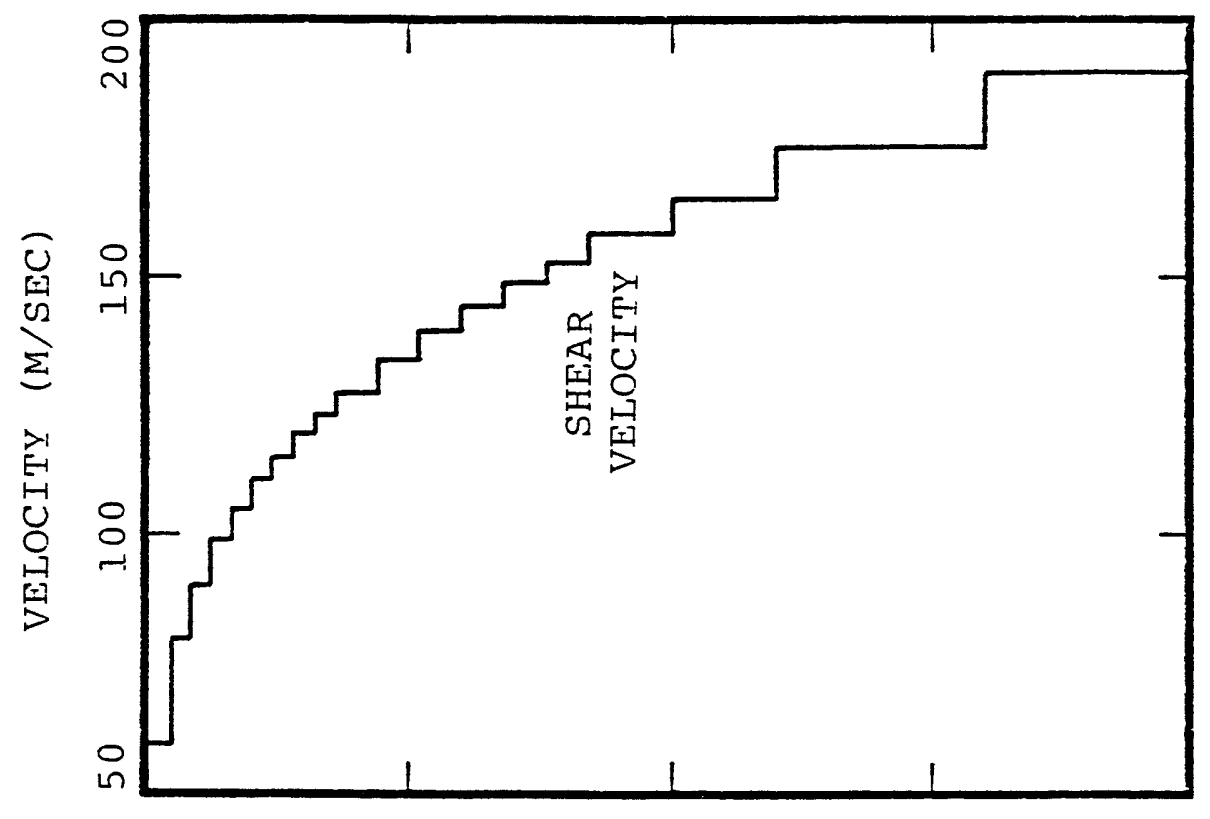

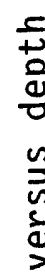

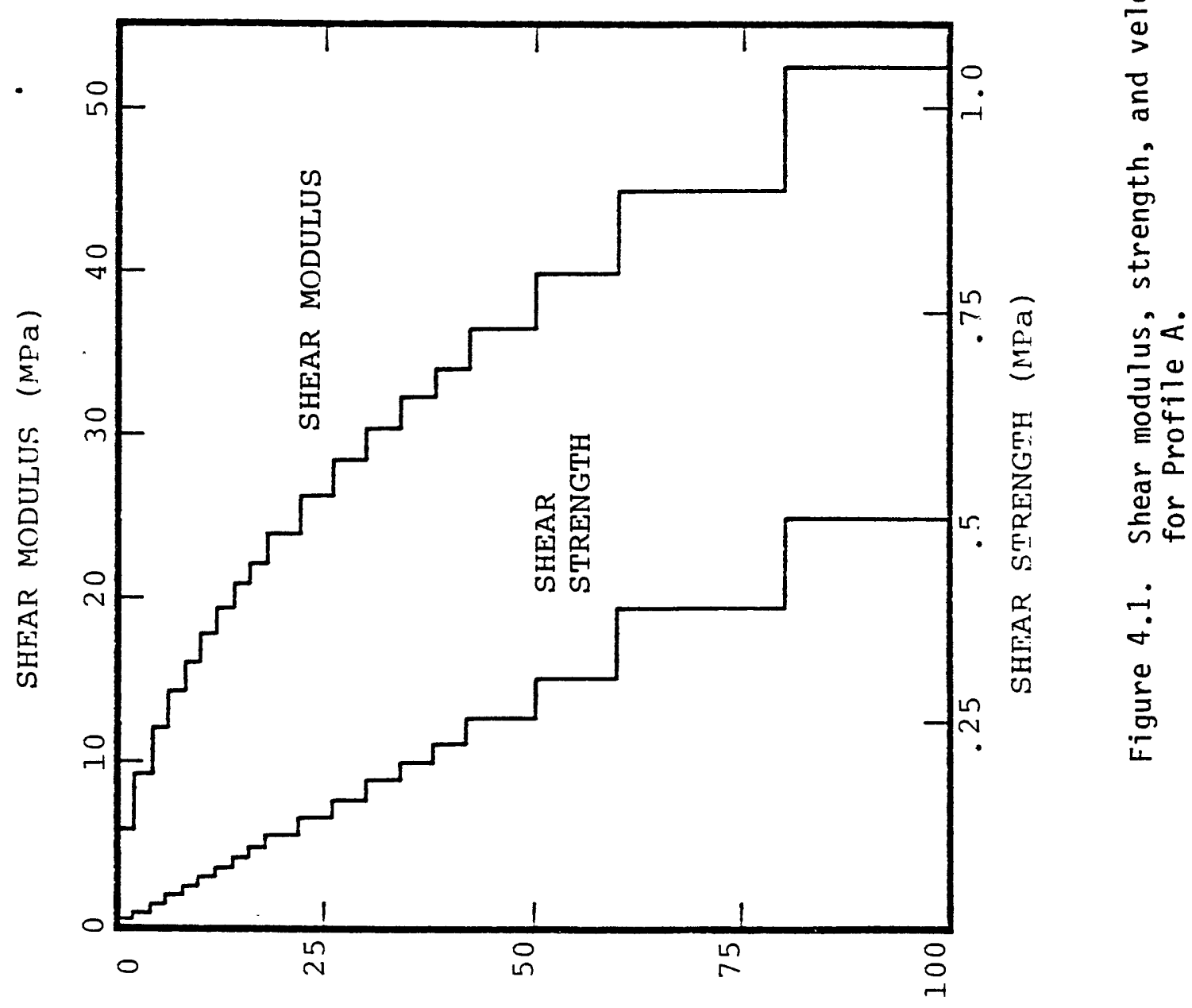

(iii) H山d 


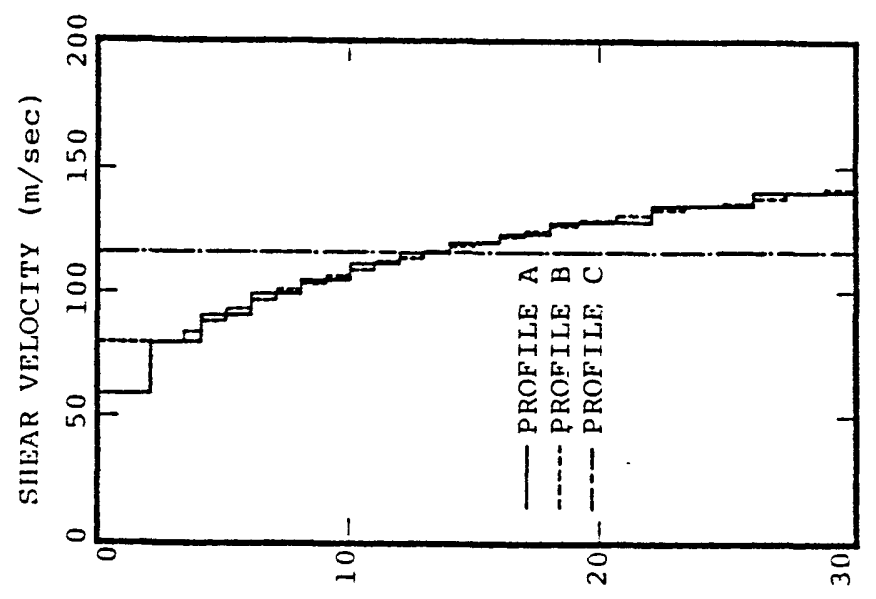

(4) HLdaO

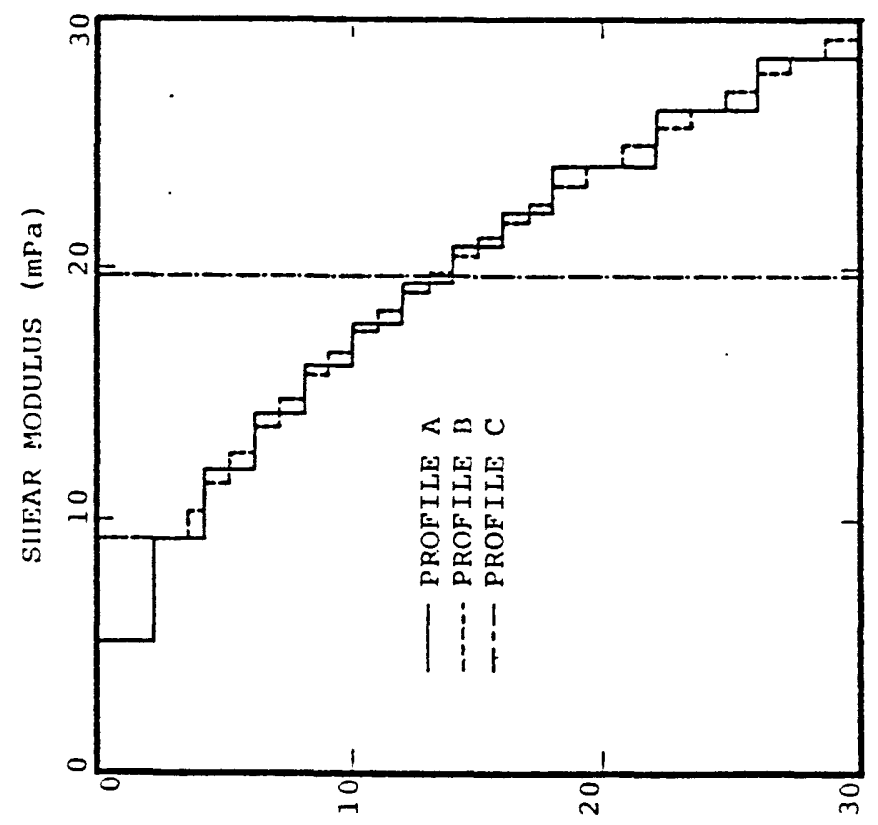

0
0
$\frac{1}{4}$
0
$\frac{1}{2}$
+7
$\frac{7}{0}$
0
0

믐

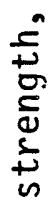

孚

ฮั

in

$4 \ll$

등 -

in 4

है ह

号

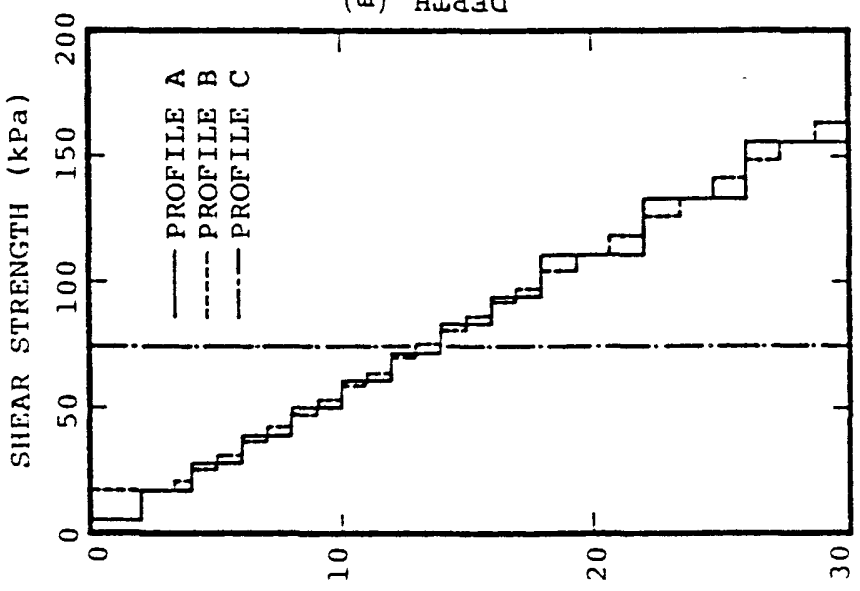

(ui) HIdJO 
disadvantage of using recorded motion, since our model of soil response assumes that the disturbance in the soil propagates as a shear wave.

A principal advantage of using theoretically derived driving motion is that the source-model and base-rock characteristics can be fixed, leading to a consistent set of distance-dependent groundmotion estimates. We can isolate the effects of surface geology and distance from the source. A second significant advantage of using synthetic input motion is that the one-dimensional study of soil response can readily be extended to two-dimensional configurations. Recorded accelerograms provide a suitable input for two-dimensional site simulations only if we prescribe the spatial character of the emergent wave field (e.g., vertically emergent shear waves); the theoretical methods, on the other hand, are capable of providing a complete description of wave type and angle of incidence. Although we did not extend our soil modeling to two-dimensions, because of computational obstacles encountered with the endochronic model (see Appendix $D$ ), a theoretical approach to specifying multi-dimensional seismic input motion for two-dimensional geologies was presented in Section 2.

\subsection{NUMERICAL METHODS}

The nonlinear soil response calculations were performed using the SWIS (Stress Waves in Solids) code (Frazier and Petersen, 1974), modified to accommodate the endochronic constitutive model. SWIS is a finite element code for the transient analysis of one-, two-, and three-dimensional, linear or nonlinear continua. The corresponding analyses with the equivalent linear technique were performed with the SHAKE code (Schnabel, et al., 1972), which is based on the Haskell matrix method (Haske11, 1953) for plane-layered media. 


\subsubsection{Nonlinear Calculations Using SWIS}

The SWIS finite element code employs a lumped-mass, explicit time-stepping method to integrate the equations of motion of a continuum. A stiffness matrix is not assembled and stored; elementcentered stresses are stored and updated from strain-rates, and nodal restoring forces are computed by one-point quadratures. In fact, for one-dimensional calculations, the method is indistinguishable from that of explicit, Lagrangian finite difference codes. Details of the numerical method are given in Frazier and Petersen (1974), and here we discuss only the use of artificial viscosity, the specification of the incoming motion, and the numerical treatment of the endochronic constitutive model.

The numerical method accurately propagates frequency components with wavelengths greater than roughly 8 to 10 element dimensions. Shorter wavelengths become significantiy dispersed as a consequence of the discretization, and, unless damped out, spurious high-frequency oscillations result and eventually contaminate the numerical solution. Such high-frequency contamination would be particularly damaging because of the memory, and ratcheting capability, of the endochronic theory; furthermore, from a computational efficiency standpoint, each strain-rate reversal adds a term to the constitutive expression, as reference to Equation 3-6 will reveal.

The explicit integration method does not inherently damp the short wavelength components of the numerical solution (aside from the hysteretic damping present in the endochronic model). For this reason, artificial viscosity was introduced into the algorithm. The artificial viscosity leads to an attenuation mechanism for harmonic elastic waves proportional to frequency squared. Using a minimum element dimension of 0.5 meters at the top of the soil column described in Figure 4.1, for example, increasing to 1.6 meters at the base, an artificial viscosity can be assigned which is sufficient to suppress virtually all spurious oscillations without significantly attenuating frequencies lower than $10 \mathrm{~Hz}$. Thus, for frequencies of 
interest, the artificial viscosity in the calculation does not significantly disturb the rate-independent properties of the endochronic constitutive model.

The introduction of the incoming motion at the base of the soil column, as well as the transmitting boundary condition which simulates the presence of a base-rock halfspace, were treated by a straightforward modification of the scheme employed by Joyner and Chen (1975), and we refer the reader to their paper for details. In their scheme, the transmitting boundary coincides with the soil-bedrock interface, and the upgoing wave motion is introduced by superimposing an appropriate traction on the transmitting boundary. In our variant, the uppermost part of the bedrock is represented by the lowermost two elements in the finite element column and was terminated below by a transmitting boundary; the upgoing wave was generated by appropriate body forces applied at the top of the bedrock. This modification was introduced for convenience and does not alter the efficacy of the scheme.

The endochronic simple shear model discussed in Section 3.2.1 requires solution of a set of simultaneous, nonlinear equations (Equations 3-2, 3-3, and 3-6) for each finite element at each time step. Given the shear strain increment $d_{\gamma}$, we use an iterative procedure based on Newton's method to solve for the intrinsic time increment $d z$ such that both Equations 3-2 and 3-6 return the same shear stress increment $d s$. That is, we seek zeroes of the function $I(d z)$ :

$$
\begin{aligned}
I(d z) & =G\left[d_{\gamma}-2 \operatorname{sgn}\left(d_{\gamma}\right) d z\right]-H(z+d z) \quad[\operatorname{Erf} \sqrt{k(z+d z)} \\
& \left.\left.-2 \sum_{n=1}^{N}(-1)^{n+1} \operatorname{Erf} \sqrt{k\left(z+d z-z_{n}\right.}\right)\right]+H(z) \mid \operatorname{Erf}(\sqrt{k z}) \\
& \left.-2 \sum_{n=1}^{N}(-1)^{n+1} \operatorname{Erf} \sqrt{k\left(z-z_{n}\right)}\right] \\
& =0
\end{aligned}
$$


where $H(z)$ and $k$ are as defined in Section 3.2.1, $z$ is the value of intrinsic time at the previous time step, $z_{n}$ is the intrinsic time associated with the $n^{\text {th }}$ strain-rate reversal, and $N$ is the number of reversals in the sign of the strain-rate which have occurred in an element. Once we have obtained $\mathrm{dz}$, we can calculate ds from Hooke's Law:

$$
d s=G\left[d_{\gamma}-2 \operatorname{sgn}\left(d_{\gamma}\right) d z\right]
$$

The function $I(d z)$ has infinite slope at $d z=0$ whenever a strain-rate reversal occurs. In order to obtain a well-behaved numerical scheme, we make a change of variable in Equation 4-1 from $d z$ to $w$, where $w=\sqrt{d z}$. The modified equation for $w$ is then more readily solved by Newton's method, and adequate accuracy is generally acquired in 2 or 3 iterations.

The summations in Equation 4-1 involve a term for each strain-rate reversal which has occurred in the element; it is also necessary to. store the intrinsic time $z_{n}$ associated with each reversal. These features require considerable computation time and computer storage, and we have invoked two simplifications in an effort to control the length of these series. First, we note that the error functions in the series "saturate" to the value 1 for large. values of the argument. If sufficient plastic strain accrues in an element, the leading terms can be successively replaced by \pm 2. Unfortunately, this simplification has proven to be of minimal utility in the present calculations, which involve complex loading histories, sometimes exhibiting many cycles of nearly elastic loading and unloading.

The second simplification makes use of the observation that successive terms in the series have opposite signs, and that the derivative of each term is a monotonically decreasing function of the argument. If the difference $z_{i+1}-z_{i}$ is small compared to $\left(z-z_{j+1}\right)^{1 / 2}$, the $i$ and $i+1$ terms can be neglected, since differentiation of the error function leads to the approximation 


$$
\begin{aligned}
& \operatorname{Erf} \sqrt{k\left(z-z_{i+1}\right)}-\operatorname{Erf} \sqrt{k\left(z-z_{i}\right)} \\
\approx & -\sqrt{\frac{k}{\pi}} e^{-k\left(z-z_{i+1}\right)}\left(z-z_{i+1}\right)^{1 / 2}\left(z_{i+1}-z_{i}\right) .
\end{aligned}
$$

The criterion that the $i$ and $j+1$ terms are deleted whenever ( $z$ $\left.-z_{i+1}\right)^{1 / 2} \gg\left(z_{i+1}-z_{j}\right)$ has been used effectively in the finite element calculations and substantially reduces the computational effort and required storage. This criterion corresponds to the physical notion of "fading memory," i.e., the material loses memory of smal1, nearly elastic hysteresis loops once they have been succeeded by significant amounts of plastic strain. Generally, it was found in practice that retention of 10 to 20 reversal terms was sufficient. An exception was the uppermost element; the free surface condition resulted in low strains in this element, and 20 to 30 reversal terms had to be retained to achieve the desired accuracy.

Appendix C lists the FORTRAN subroutine for the endochronic constitutive model of simple shear of dry sand which was used in the present study in conjunction with the SWIS finite element code.

\subsubsection{Equivalent Linear Calculations with SHAKE}

The computer code SHAKE, (Schnabel, et. al., 1972) was used in this program for the equivalent linear calculations. The code computes the response induced in a system of homogeneous, viscoelastic layers, of infinite horizontal extent, by a vertically travelling shear wave. Acceleration, strain and stress histories are first computed in the frequency domain, using interlayer displacement and stress compatibility conditions, and are then transformed to the time domain using a fast Fourier Transform algorithm.

The soil profile motion is driven by an acceleration history, the object motion, that can be applied to any layer. After being transformed to the frequency domain all contributions to the object motion above a user specified maximum frequency are removed. The 
SHAKE code assumes strain dependent material properties. Starting with an initial guess for layer shear moduli and critical damping ratios, the code iterates until the material properties at the beginning of an iteration correspond to the strains predicted at the end of the iteration; typically no more than five iterations are necessary. The SHAKE code accounts for the depth dependence of soil shear moduli by assuming

$$
G=G_{R}\left(\sigma_{m} / \sigma_{R}\right)^{1 / 2}
$$

where

$$
\sigma_{m}=\sigma_{v}\left(1+2 k_{0}\right) / 3, \quad \sigma_{v}=\rho g d .
$$

Here $\sigma_{m}$ is the soil confining pressure, $\sigma_{v}$ is the vertical stress caused by soil overburden, $k_{0}$ is the user defined coefficient of earth pressure and $\sigma_{R}$ is the reference confining pressure at which $G$ equals $G_{R}$. In the present study

$$
K_{0}=0.4, \sigma_{R}=114.9 \mathrm{kPa} \text {. }
$$

Before SHAKE could be used in this investigation, two coding errors had to be corrected. As received, SHAKE calculated stress histories by multiplying strain histories by the elastic shear modulus; thus, computed stress-strain histories for cyclic loading exhibited no hysteresis. This was corrected by identifying the stress history with the inverse fast Fourier transform of the product of complex shear modulus, Equation (3.9), and the complex-valued strain Fourier components. The other, less obvious, error was incorrect dynamic dimensioning of the temporary storage array $A A$, the consequence of which was that some fast Fourier transformation information computed at the beginning of program execution was inadvertently set to zero before being used to compute stress and strain histories. This error was corrected in the main routine by adding 8 to the variable NS so that it was defined as

$$
N S=N A A+2(\text { NAMAX }+4)
$$


A total of six analyses was performed, corresponding to problems 1 to 3 and 6 to 8 of Table 4.1. Layer thicknesses used to model the $100 \mathrm{~m}$ soil profile are shown in Figure 4.3. The number of layers is the maximum permitted in SHAKE. For the $10 \mathrm{~m}(30 \mathrm{~m})$ analyses, the layering was identical to the top $10 \mathrm{~m}(30 \mathrm{~m})$ of the $100 \mathrm{~m}$ profile. In each analysis the object motion was an acceleration history consisting of 1000 values at $0.01 \mathrm{sec}$ intervals. However, all components for frequencies greater than $20 \mathrm{~Hz}$ were discarded to reduce computation time. In all calculations, removal of the high frequency Fourier component changed the peak input acceleration by no more than 1.5 percent.

\subsection{NUMERICAL RESULTS}

Figures 4.4 and 4.5 show acceleration and velocity waveforms at the surface of the soil for several of the nonlinear studies. Figure 4.4 displays the effects of soil thickness on these waveforms, and Figure 4.5 depicts the different character of the surface motion at the three site-to-fault distances. One effect of increasing the soil thickness is to lengthen the characteristic period associated with the peak acceleration and velocity. A second effect is, of course, to lengthen the resonance period governing the latetime reverberations. The waveform differences evident in Figure 4.5 among the three receiver locations are largely associated with the differences in bedrock motion discussed in Section 2; directivity, and anelastic and geometric attenuation along the propagation path from the source, are largely responsible for the differences in frequency content and amplitude.

Table 4.1 summarizes the peak motions (acceleration and velocity) obtained from the present analyses. Note that in all cases except Problem 8, the nonlinear analyses give soil site peak accelerations which are significantly smaller than the corresponding rock site peak acceleration. Furthermore, the equivalent linear 


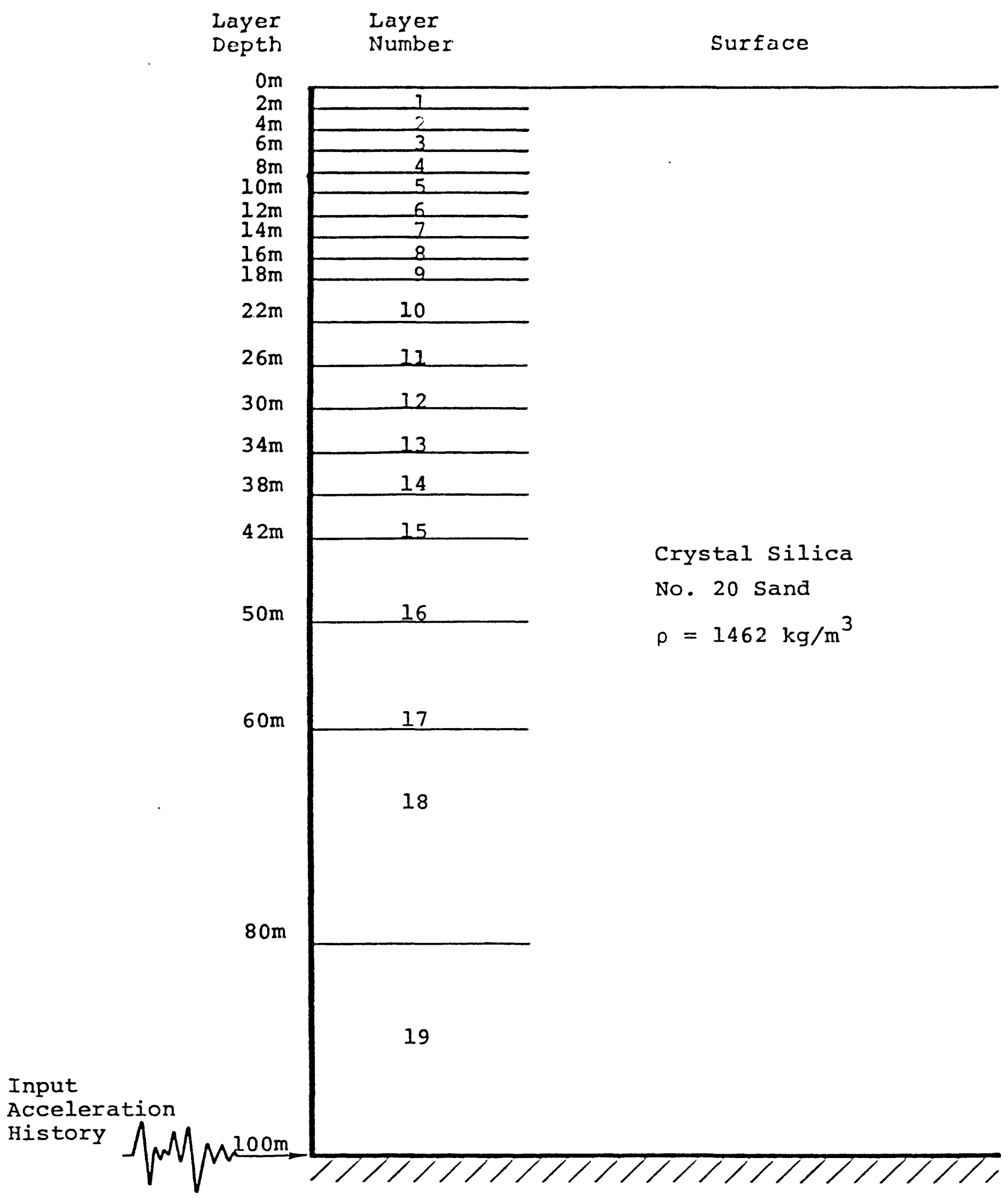

Fiqure 4.3. Soil layering used in SHAKE calculations. 

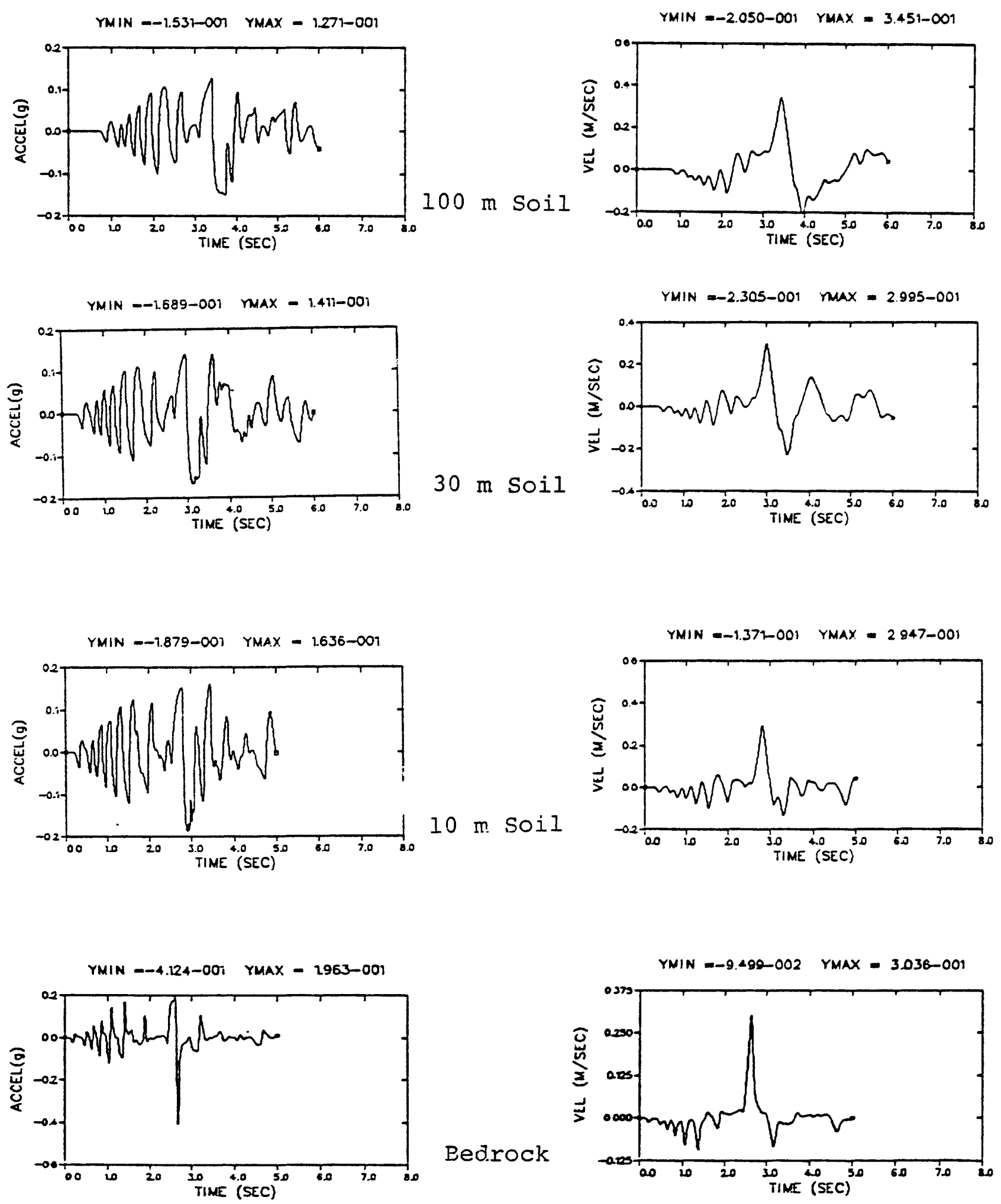

Fiqure 4.4. Computed surface acceleration and velocity time histories for three different soil deposit thicknesses (Problems 1,2 , and 6). Also shown is the bedrock response. Distance from the fault in each case was 5 kilometers. 

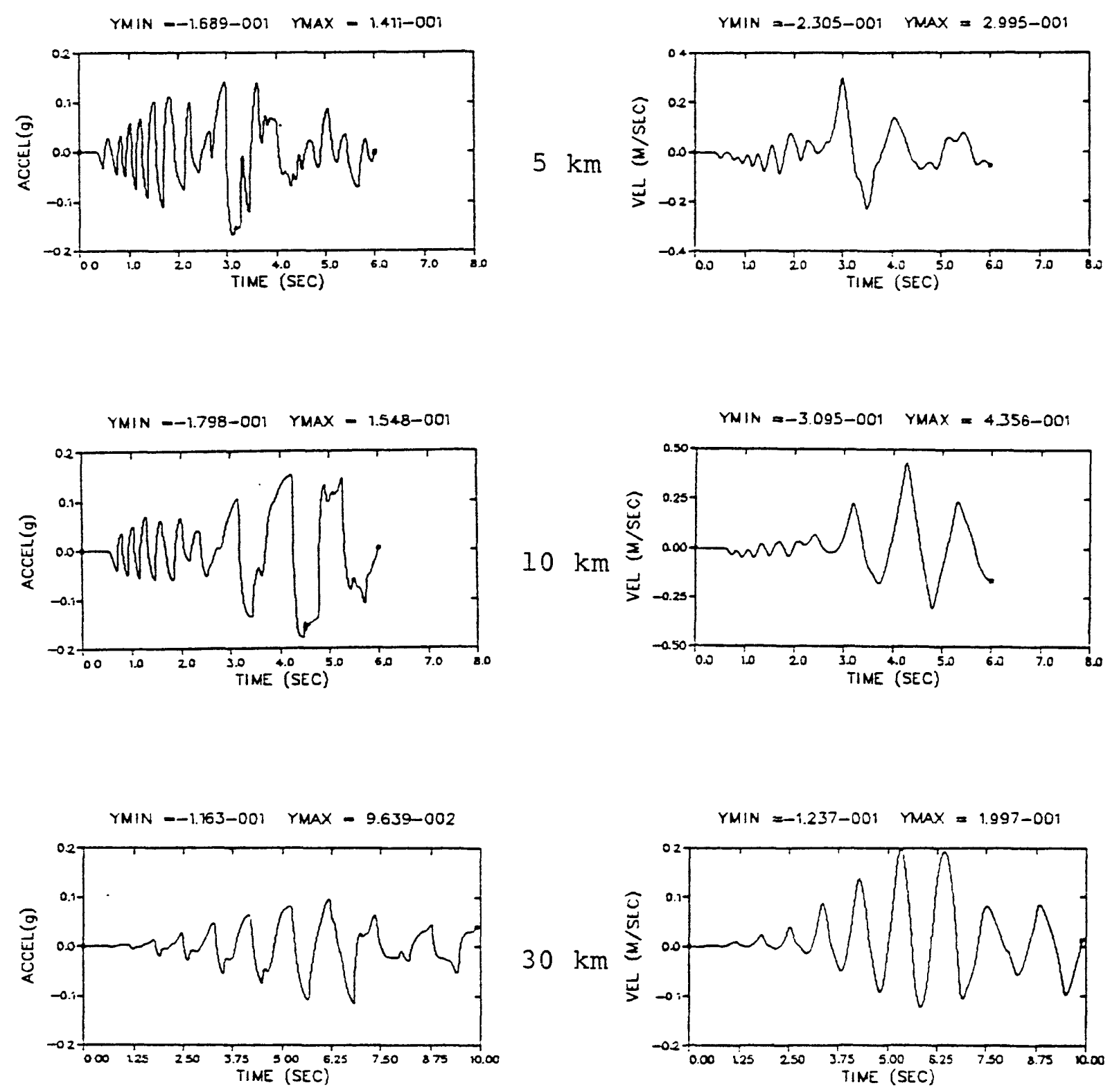

Fiqure 4.5. Surface acceleration and velocity time histories computed for 30 meter deep soil sites at 5, 10, and 30 kilometer distance from source. (Problems, 6, 7 and 8 ). 
method substantially overestimates peak accelerations compared with the nonlinear analyses, again excepting Problem 8. Peak velocity is also overestimated by the equivalent linear method, though by a smaller amount.

In this section we analyze the results of the nonlinear calculations in detail and compare them with the equivalent linear results. Section 4.4 .1 examines the constitutive behavior of the endochronic model in the nonlinear calculations. Section 4.4.2. examines the sensitivity of the nonlinear results to details of the soil profile; results of Problems 4, 5, and 6 representing Profiles $B, C$, and $A$ respectively, are compared. Section 4.4 .3 compares the calculated peak motion obtained by our analytical method with empirical peak motion relationships derived from earthquake ground motion data. Section 4.4.4. compares the nonlinear results to those obtained by the equivalent linear method.

\subsubsection{Peformance of the Endochronic Constitutive Model}

The endochronic model employed in this study has perhaps the most advanced capabilities for modeling the hysteretic behavior of soils of any of the existing soil models. Within the context of simple shear, to which the current study is limited, the modeling capability of the theory includes hardening, cyclic creep (ratcheting) and elastic behavior for infinitesimal unloading or reloading following a strain-rate reversal. The latter capability was absent from earlier versions of the endochronic theory, and led to criticism of the theory's suitability for numerical wave propagation calculations (Sandler, 1978). The new theory, used in the present study, does not, however, suffer from this objection, and to demonstrate the excellent behavior of this model in wave propagation studies, the details of computed stress-strain paths are provided.

Figures 4.6 through 4.11 show the stress-strain paths computed at several different depths in the soil deposit for the six nonlinear calculations performed for Profile A -- Problems 1 through 3 and 6 through 8. Triangles on the curves delimit 0.5 second time 

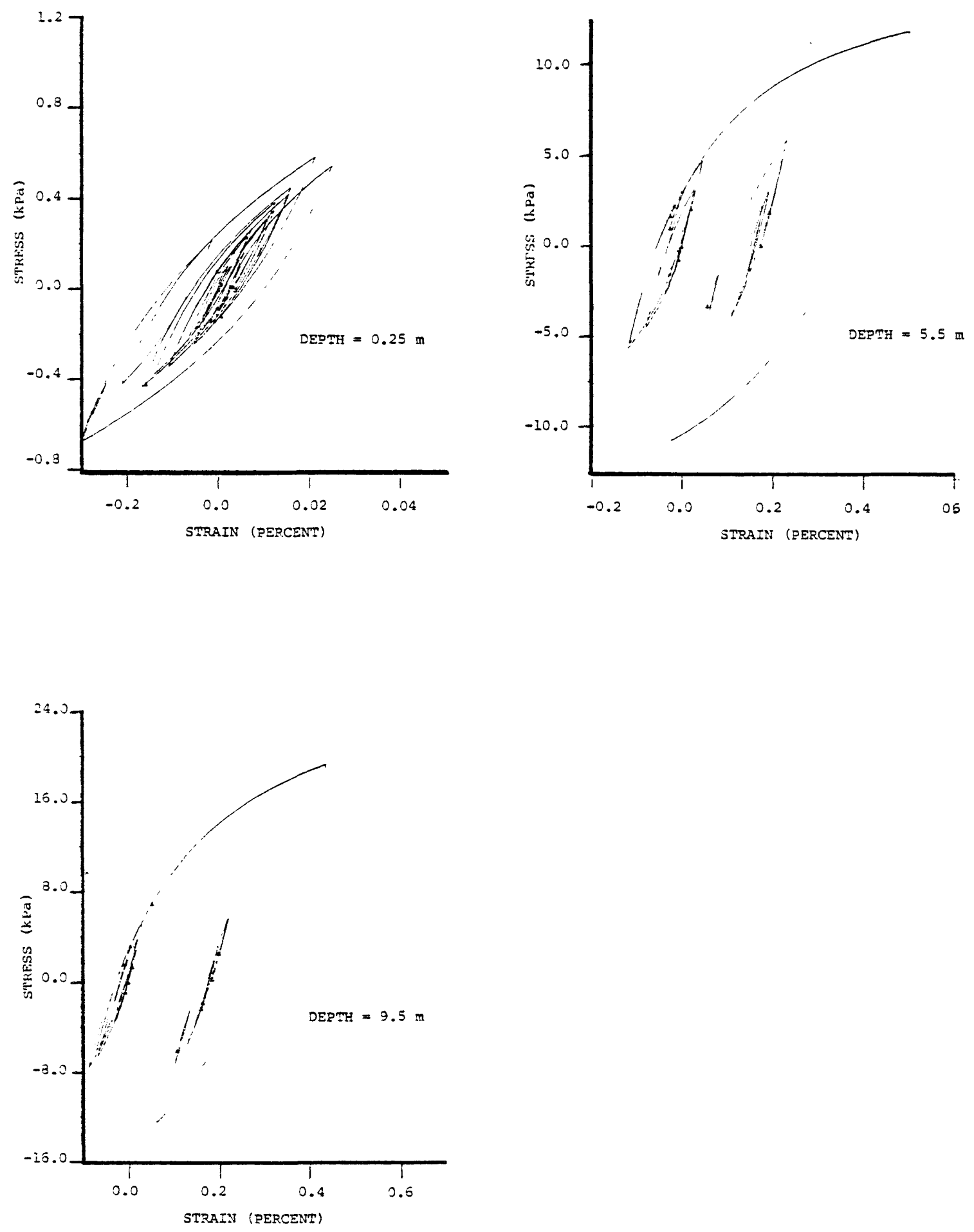

Fiqure 4.6. Stress-strain paths predicted by the endochronic model at several depths in the soil deposit (Problem 1). 

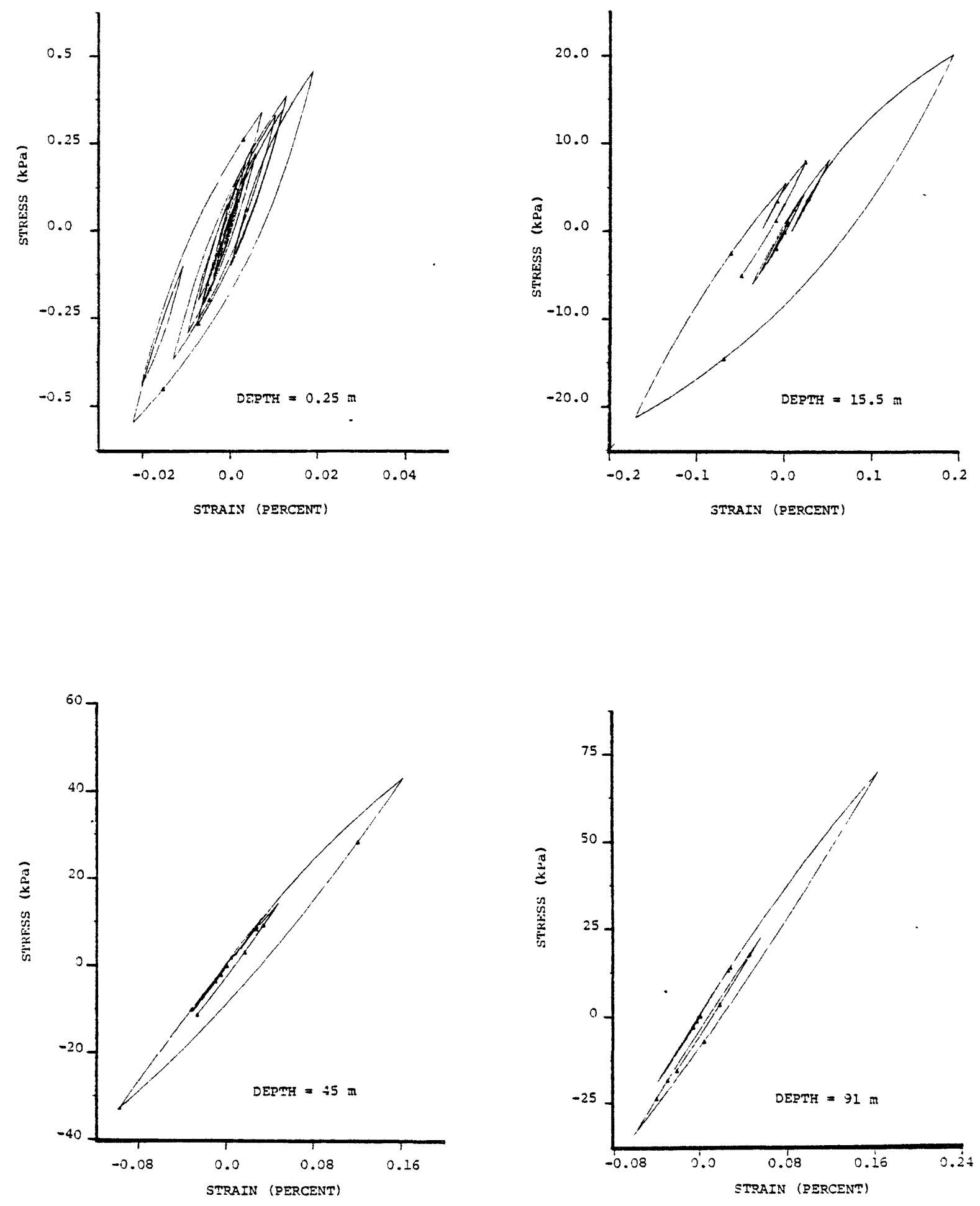

Fiqure 4.7. Stress-strain paths predicted by the endochronic model at several depths in the soil deposit (Problem 2). 

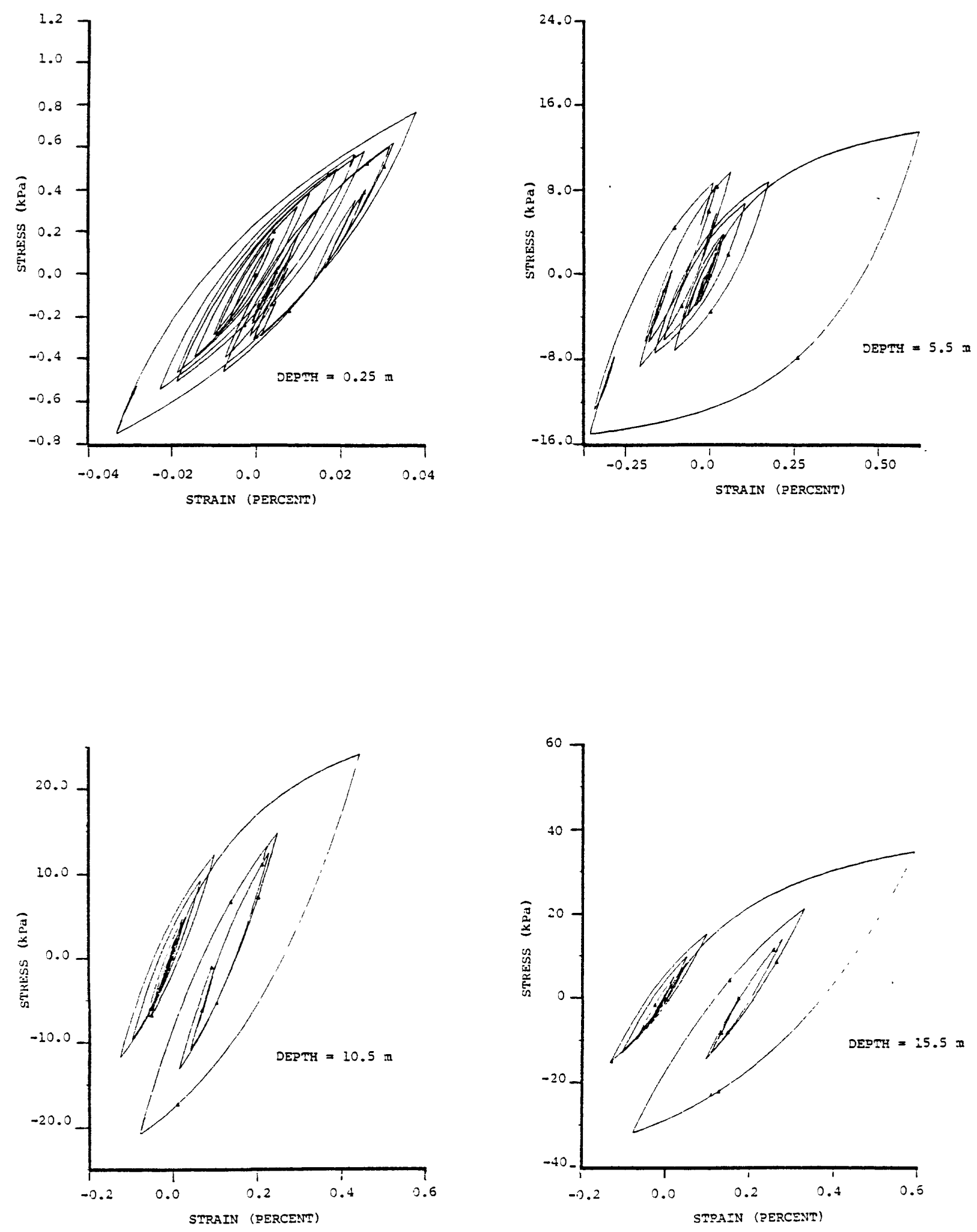

Fiqure 4.8. Stress-strain paths predicted by the endochronic model at several depths in the soil deposit (Problem 3). 

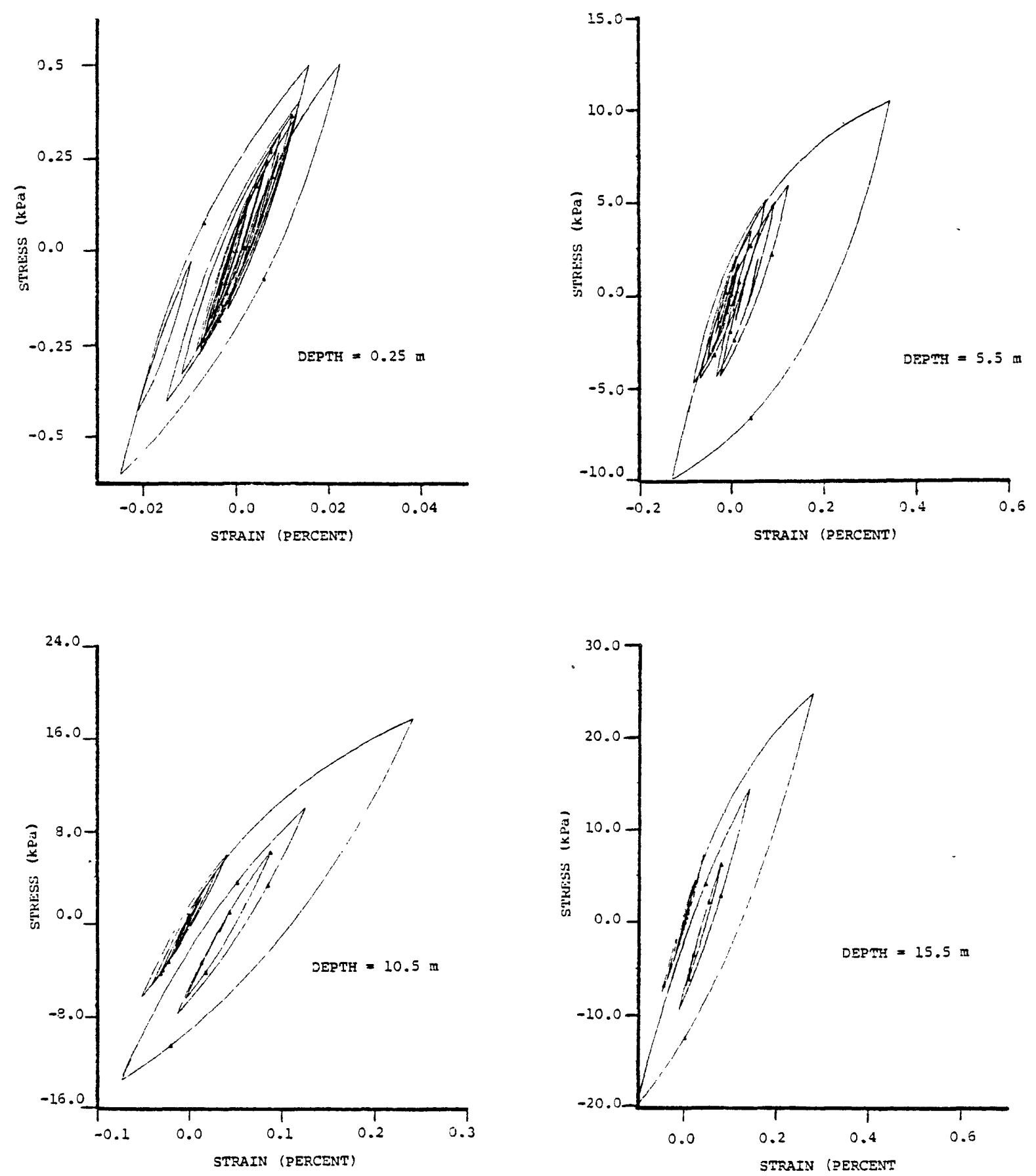

Fiqure 4.9. Stress-strain paths predicted by the endochronic model at several depths in the soil deposit (Problem 6). 

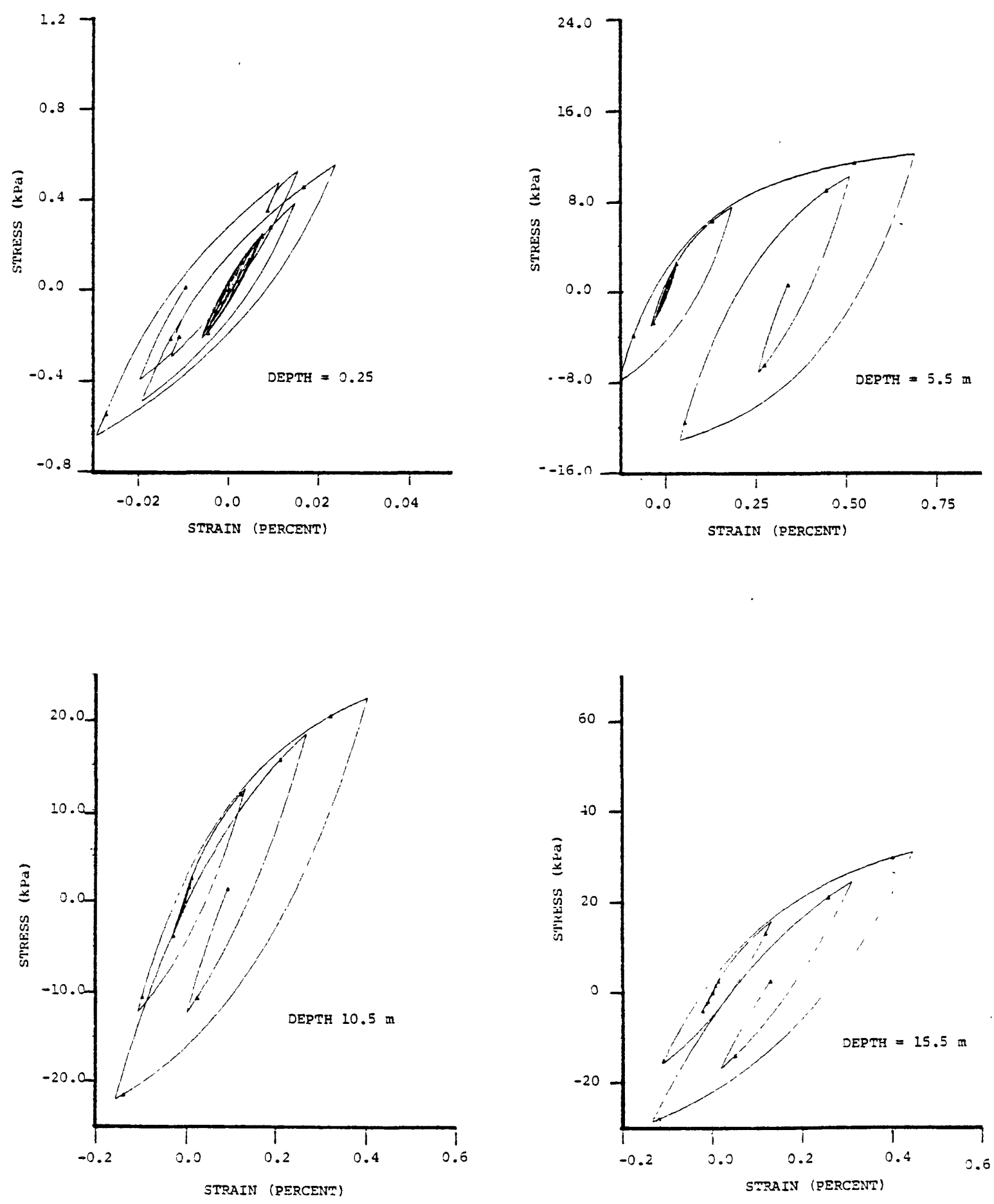

Fiqure 4.10. Stress-strain paths predicted by the endochronic model at several depths in the soil deposit (Problem 7). 

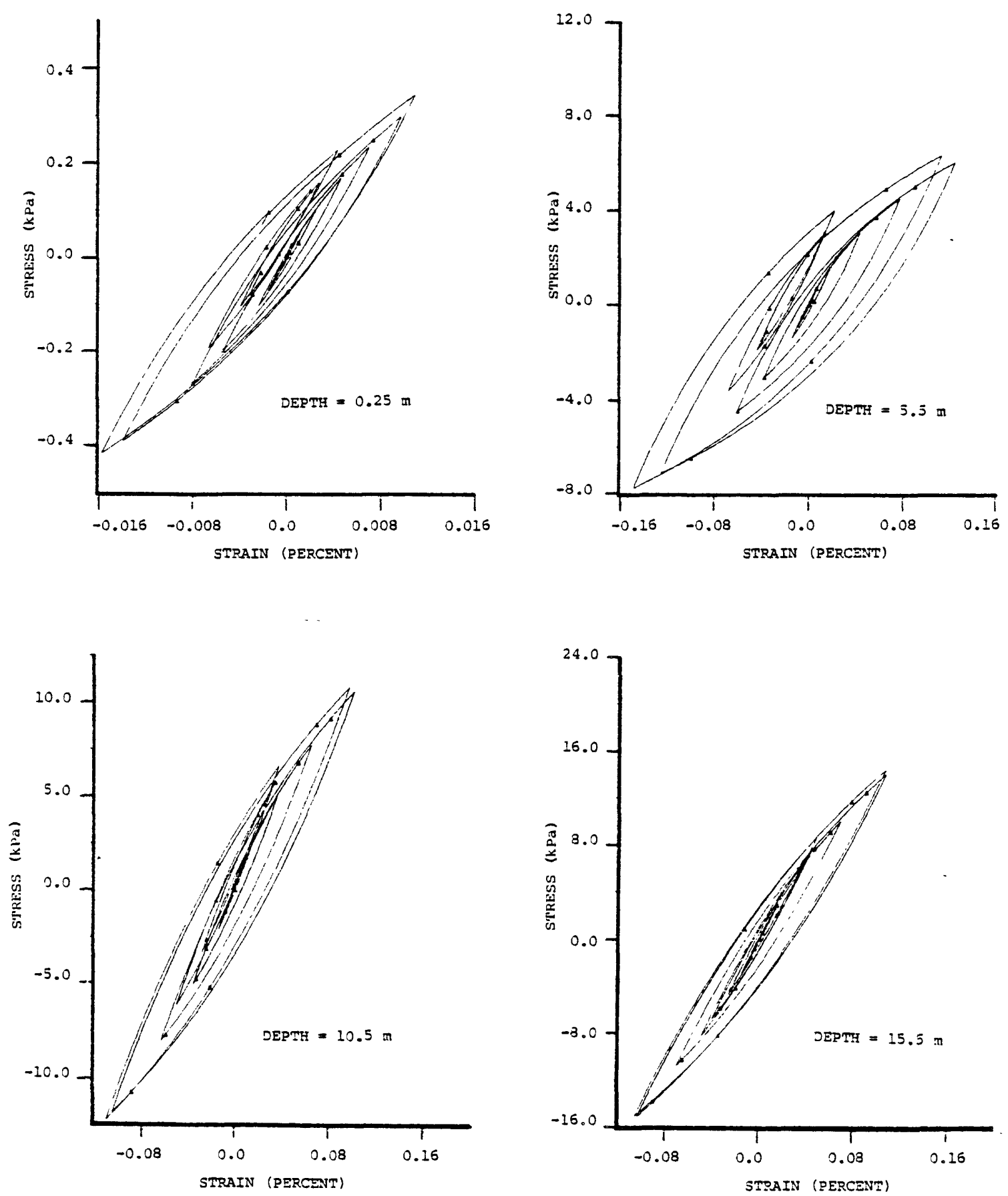

Fiqure 4.11. Stress-strain paths predicted by the endochronic model at several depths in the soil deposit (Problem 8). 
intervals. Taken together, these figures verify two anticipated features of the model. First of all, loading and unloading indeed invariably initiate along a slope corresponding to the shear modulus. Second, all hysteresis loops close.

A third feature of the endochronic model evident in these figures relates to the absence of abrupt "corners" in the stressstrain paths. Pyke (1979) has discussed several inadequacies associated with those soil models satisfying a generalized set of Masing criteria, when subjected to irregular, transient loading. This category would include many standard soil models, including the Iwan class of models. In particular, Pyke noted that under irregular loading, these models exhibit sharp corners in the stressstrain path, in conflict with experimental results. This phenomenon occurs in Iwan-type models whenever a loading or unloading curve intersects a previous loading or unloading curve. Figures 4.6 through 4.11 demonstrate that such unrealistic behavior is not displayed by the endochronic model; discontinuities of slope occur only at points of loading rate reversal, in agreement with observations.

Figure 4.7 shows that energy dissipation, as indicated by hysteresis loop area, is greater at shallow depths than it is near the center and base of the soil deposit. The four subsequent figures each give results at $0.25,5.5,10.5$, and 15.5 meters depth, and among these depths, the greatest dissipation in each case apparently occurs at 5.5 meters depth. It will be seen in Section 4.4.4. that this is approximately the depth above which the peak motion estimates from the equivalent linear method diverge from those of the nonlinear method.

A particularly vivid illustration of hardening occurs in Figure 4.8. There are several cycles of motion between stress limits of approximately $\pm 8 \mathrm{kPa}$, followed by a large, highly dissipative strain cycle, followed by several more cycles between \pm $8 \mathrm{kPa}$. The secant modulus associated with the latter cyclic motion 
is nearly 50 percent greater than that of the initial cyclic motion, thereby illustrating the significant hardening that occurs through the deformation history.

Finally, we note that the capability of the endochronic model to describe cyclic creep (ratcheting) under asymmetric loading is well illustrated, for instance, in Figures $4.6,4.8$ and 4.10 . Cyclic creep is commonly observed in soils subjected to cyciic loading about some prestressed state, and leads to an accumulation of strain with successive cyclic loading.

\subsubsection{Sensitivity to Soil Profile Details}

The depth dependences of shear modulus and shear strength deduced in Appendix $B$ for loose sand imply that both approach zero as depth below the surface decreases. Nonlinear calculations 4, 5 , and 6 for Profiles $B, C$, and $A$, respectively, based on the same input seismogram ( $5 \mathrm{~km}$ site) and soil thickness (30 meters), are compared here to delineate the sensitivity of computed motion to the details of the soil profile.

Figure 4.12 shows maximum values of shear stress and strain obtained as functions of depth for the three cases. Peak stresses for Profiles $A$ and $B$ are indistinguishable; peak stress for Profile $C$ coincides with the other two cases down to about 7 meters, then diverges somewhat. Peak strain is similar for Profiles $A$ and $B$ below about 3 meters depth; Profile $B$ gives somewhat smoother results, reflecting the smoother approximation of the shear modulus and shear strength depth dependence. At shallower depths, however, Profile $A$ results in a large strain concentration at about 2 meters depth which is absent in the calculation for Profile $B$. Peak strain for Profile $C$ is lower than for the other 2 cases, for depths above 20 meters, and decreases smoothly toward zero near the surface.

Figure 4.13 shows maximum values of acceleration and velocity as functions of depth for the three cases. Both peak acceleration and peak velocity for Profiles $A$ and $B$ are nearly identical throughout the soil column. Both peak acceleration and velocity are 

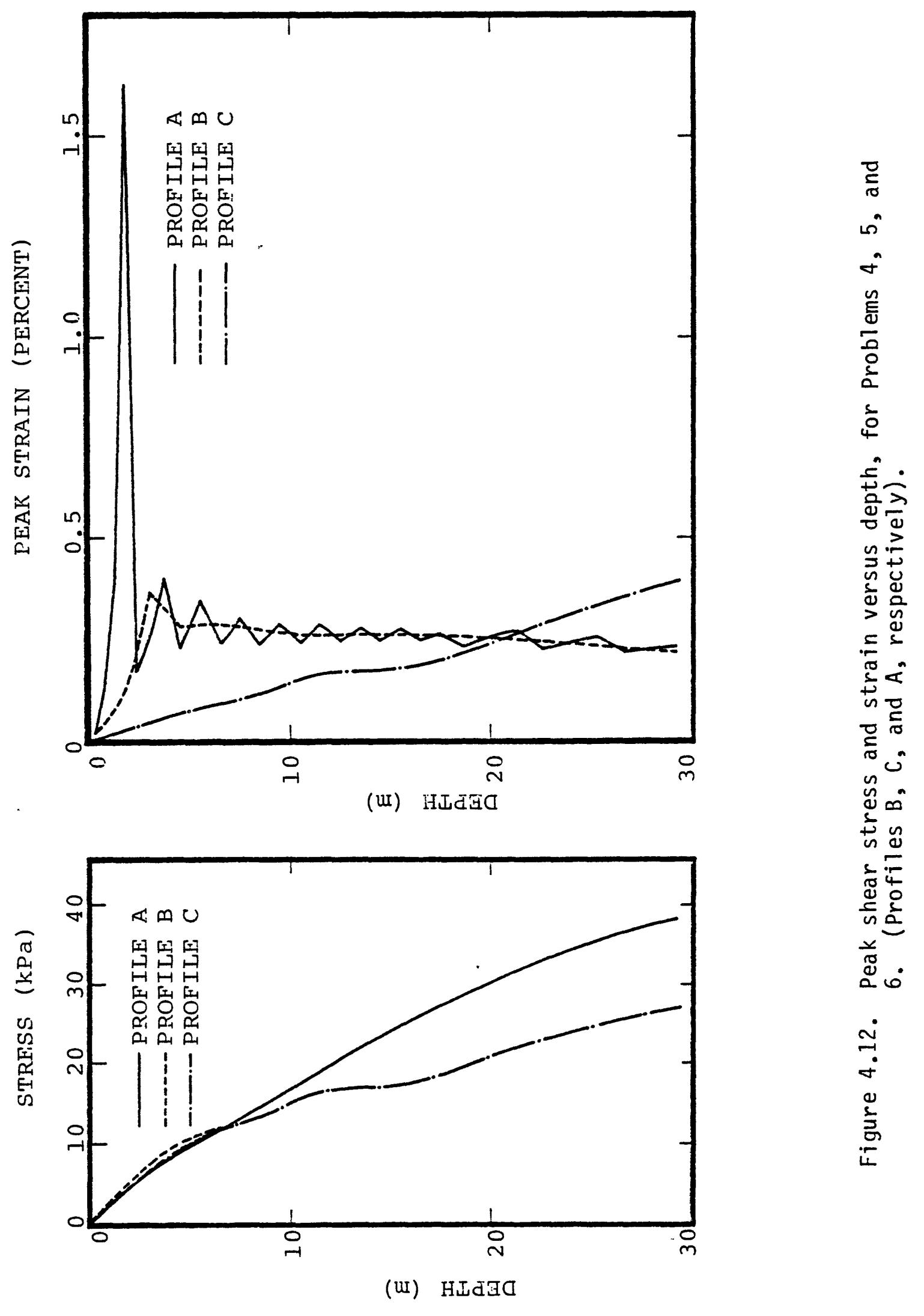


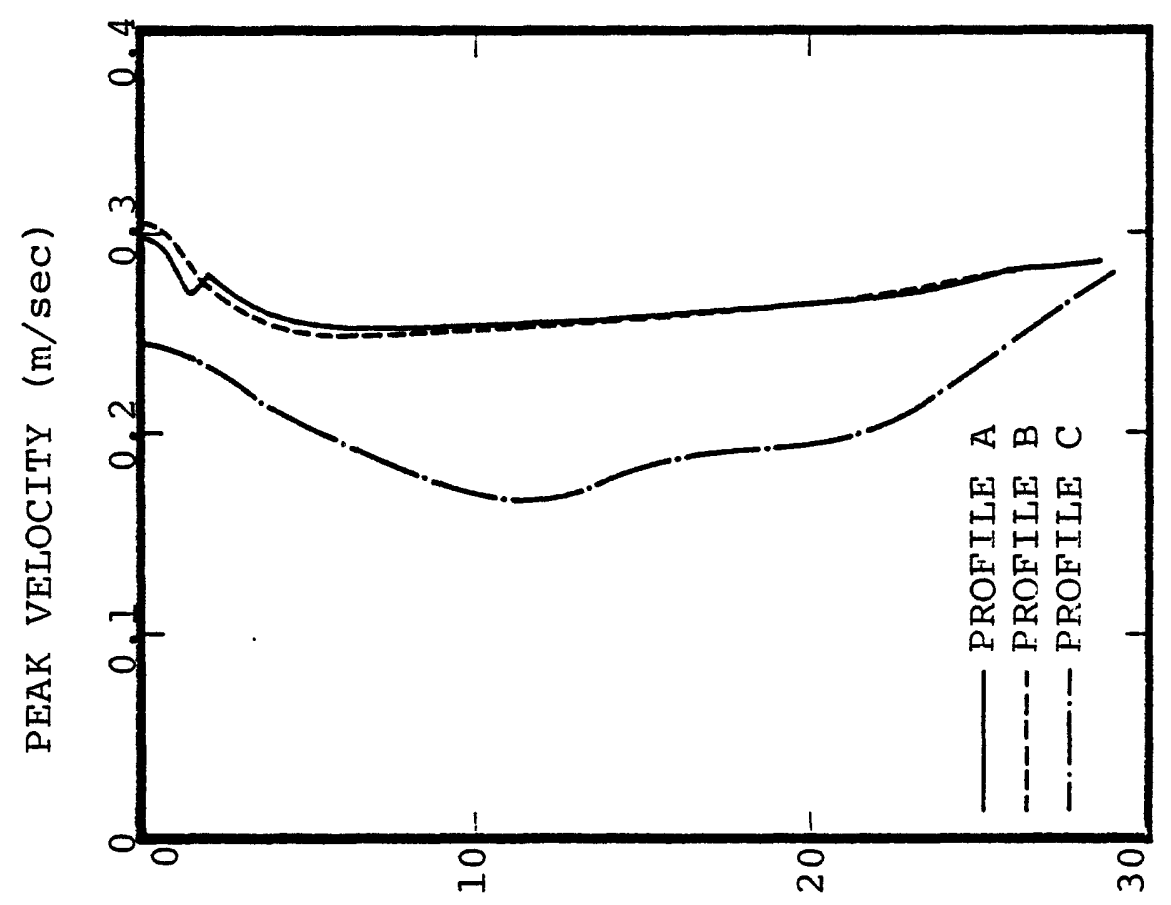

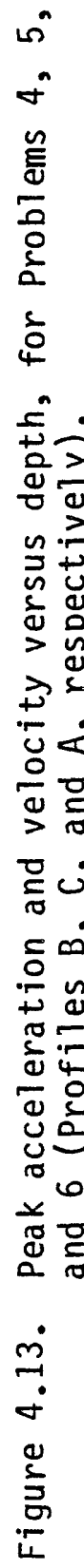

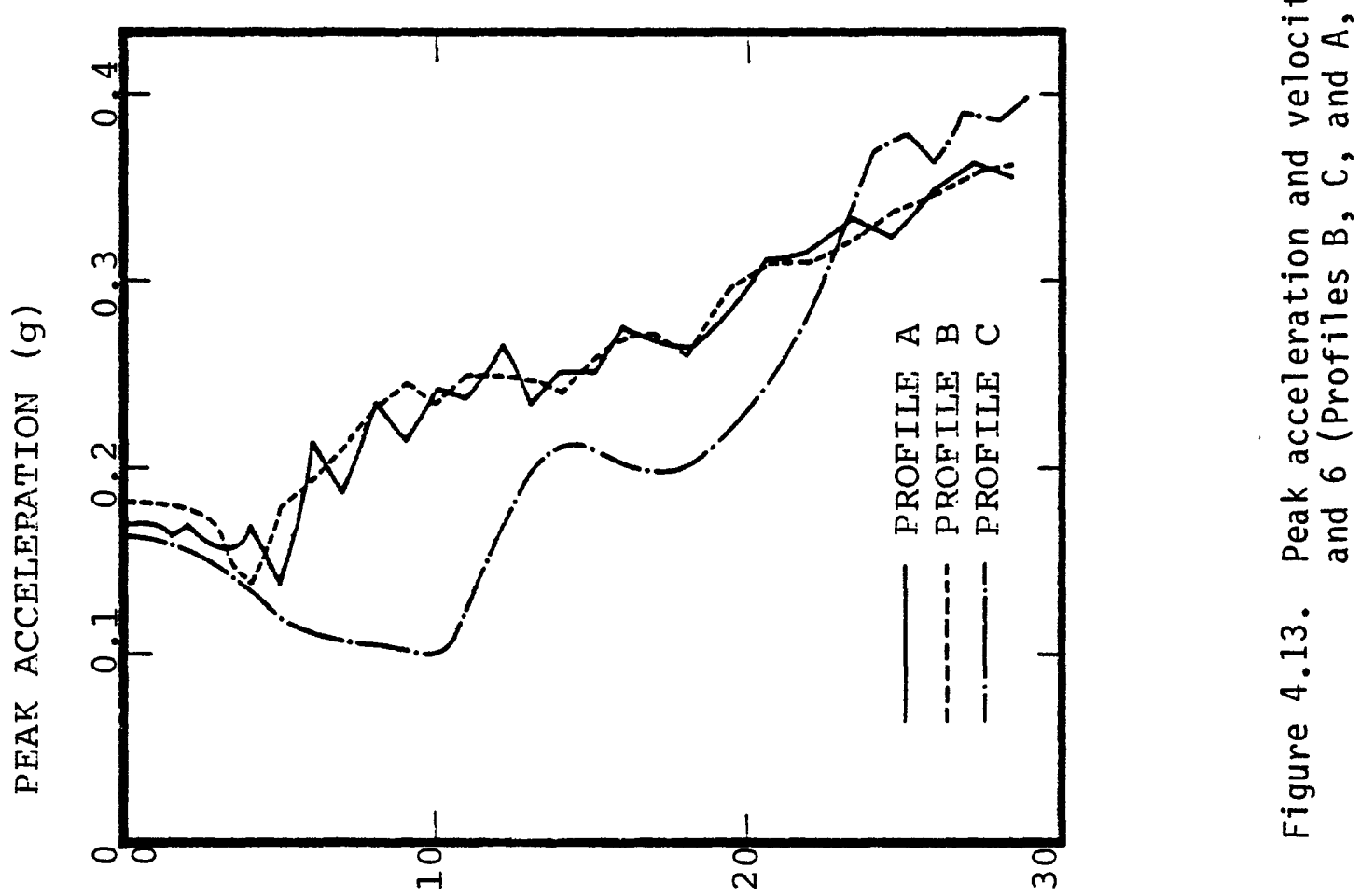

(ii) H๘dت 
somewhat lower for Profile C, particularly around 10 meters depth; at the surface, however, peak acceleration for Profile $C$ is nearly the same as for the other two cases, and peak velocity is only 20 percent lower for Case $C$. Apparently peak ground motion is not sensitive to the precise manner in which the depth dependence of shear modulus and shear strength are discretized, although maximum shear strain can be sensitive to this discretization. We have not shown comparisons of time histories of motion, but these also are very similar for all three cases, and nearly indistinguishable for Profiles $A$ and $B$.

\subsubsection{Comparison of Peak Motion Estimates with Empirical Trends}

Inferences about site-dependent ground motion are severely limited by the absence of sufficient strong motion data for distances less than 20 kilometers. It is important, therefore, that analytical methods be developed to supplement the empirical approach to ground motion prediction. The current study has focussed attention on an admittedly idealized configuration -- the source is simplified, the soil deposit is taken to be a loose, drained sand, and the P-SV wave contribution to ground motion has been neglected. In spite of these idealizations, the approach does account in a fairly consistent manner for such distance-dependent effects as anelastic attenuation, dispersion, and earthquake source finiteness; furthermore, the endochronic soil model was found to reproduce very accurately the type of behavior a cohesionless soil would exhibit under conditions of simple shear.

It is worthwhile to examine the site-dependent peak motion estimates obtained from this study to establish to what extent they are consistent with well-established empirical trends and to what extent they support the statistically marginal inferences. A number of studies have attempted to delineate the effect of site geologic conditions on peak ground motion, and on its attenuation with distance from the energy source. Duke et al. (1972) and Donovan (1973) concluded from analysis of strong motion data from the 1971 
San Fernando earthquake that, for near-field sites, peak accelerations recorded on rock are generally higher than peak accelerations recorded on soil, whereas at distances greater than 40 to 50 kilometers, soil sites experience higher peak accelerations. Seed et. al. (1976a) studied data from 8 western United States earthquakes with magnitudes of about 6.5. They similarly found that peak acceleration was higher on rock than on soil in the near-field (distance less than $\sim 40$ kilometers); furthermore, it was their finding that peak acceleration attenuates more slowly with distance for stiff soil sites than for sites on rock, and more slowly still for sites on deep, cohesionless soil. Trifunac and Brady (1976) analyzed data from 57 earthquakes in the western United States and concluded that for magnitudes less than 5 , peak accelerations are somewhat higher on rock sites than on soil sites. For magnitudes greater than 6 , on the other hand, they found significant differences in peak accelerations for the two classes of sites. The analys is of Trifunac and Brady assumed however, that the distanceattenuation curve has a prescribed shape, and is independent of site geology. If peak acceleration on soil sites actually attenuates more slowly with distance than peak acceleration on rock sites, then the different behavior observed by Trifunac and Brady for the two magnitude classifications (less than magnitude 5 and greater than magnitude 6) might simply reflect a difference in the average distance to the energy source for observations in the two magnitude classes. Finally, Boore et. al. (1980) report no statistically significant difference between soil and rock site peak acceleration for the San Fernando earthquake.

Figure 4.14 contains plots of peak acceleration and peak velocity versus distance for the three rock site computations and three 30-meter-deep soil site computations (Problems 6, 7, and 8). The solid curves are from Boore et. al. (1980) and represent the 70 percent prediction interval obtained from an analysis of strong motion data from earthquakes in the magnitude range 5.0 to 5.7. In their study, Boore et. al. limited consideration to data recorded at distances between 5 and 30 kilometers from the fault slippage, and 

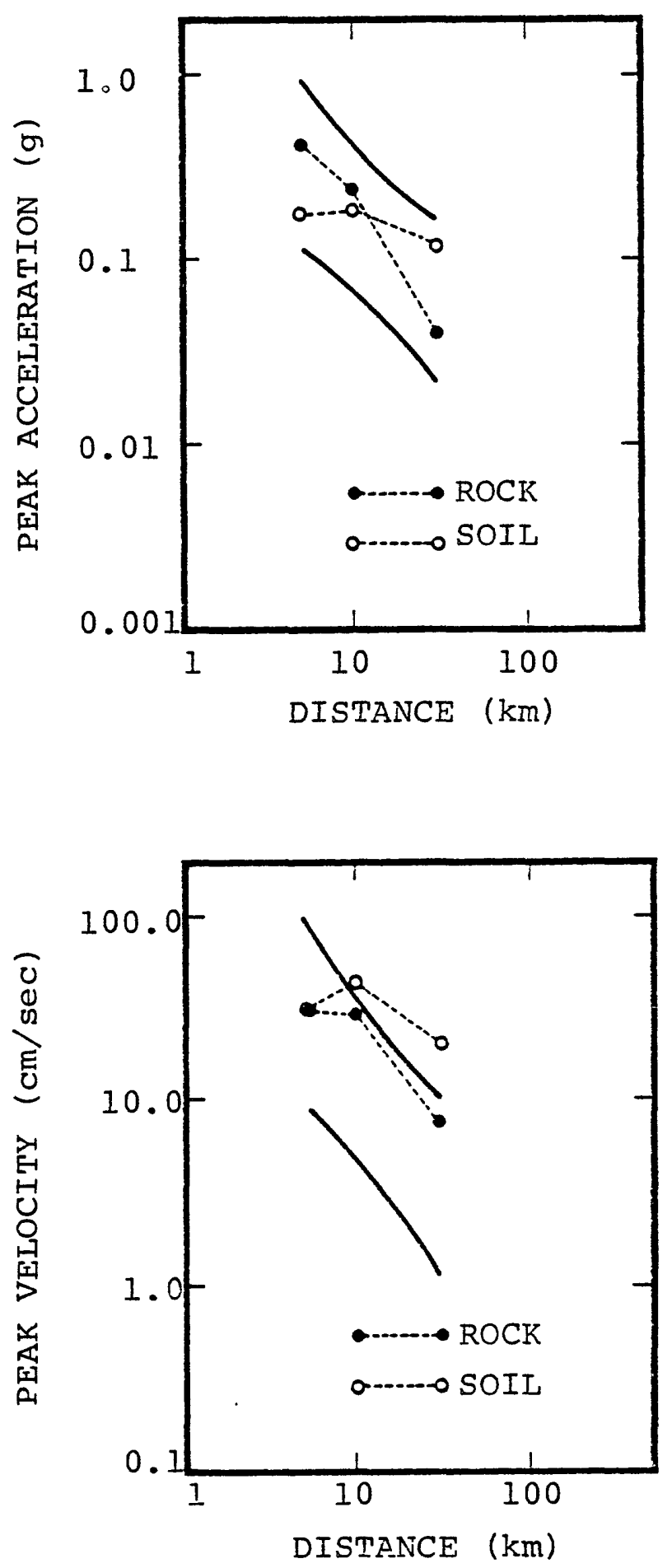

Figure 4.14. Peak horizontal acceleration and velocity, plotted versus distance to the fault. A7l six data points are for the source model and baserock structure of Section 2; solid circles are from rock-site calculations, open circles are from 30 meter deep, nonlinear soil-site calculations (Problems 6, 7, and 8 in Table 4.1.). The pairs of solid curves are the 70 percent prediction intervals obtained by Boore, et al., (1980) from an analys is of strong motion data from earthquakes in the magnitude range 5.0 to 5.7 . 
did not differentiate the data in this magnitude class according to site geology. The peak accelerations and velocities calculated on rock sites all fall within the 70 percent prediction interval of Boore et. al. Recall that the source model was constrained to give peak acceleration and velocity values at 5 and 10 kilometers consistent with the Parkfield earthquake (magnitude 5.5), so the significant point here is that the same source model yields good agreement with the empirical trends at 30 kilometers distance as well.

The nonlinear soil site calculations corroborate rather strongly the inference that peak acceleration recorded on cohesionless soil attenuates more slowly with distance than does peak acceleration recorded on rock. At 5 kilometers distance from the fault slippage, peak acceleration computed on the loose sand deposit is about 40 percent of that computed on rock, whereas at 30 kilometers distance, peak acceleration on the sand deposit exceeds that on rock by about a factor of 3 .

Figure 4.15 summarizes the comparison in another manner. The solid and dashed curves relating peak acceleration for different site conditions to bedrock peak acceleration are taken from Seed et. al. (1976a) who derived them from empirical distance attenuation curves. The curves show the tendency for low-level acceleration peaks to be progressively more amplified by increasingly softer site conditions, and for acceleration peaks in excess of 0.1 to $0.2 \mathrm{~g}$ to be progressively more attenuated by increasingly softer site conditions. The nonlinear calculations, represented by open circles, show a similar pattern; undoubtedly, the consideration of an artificially loose sand deposit in the present study has led to a strong enhancement of the trend, however, and accordingly we emphasize the qualitative agreement with the empirical trend rather than the quantitative results.

The triangles in Figure 4.15 show the corresponding results for the equivalent linear calculations. In each case, the equivalent linear model leads to a peak acceleration of soil exceeding 


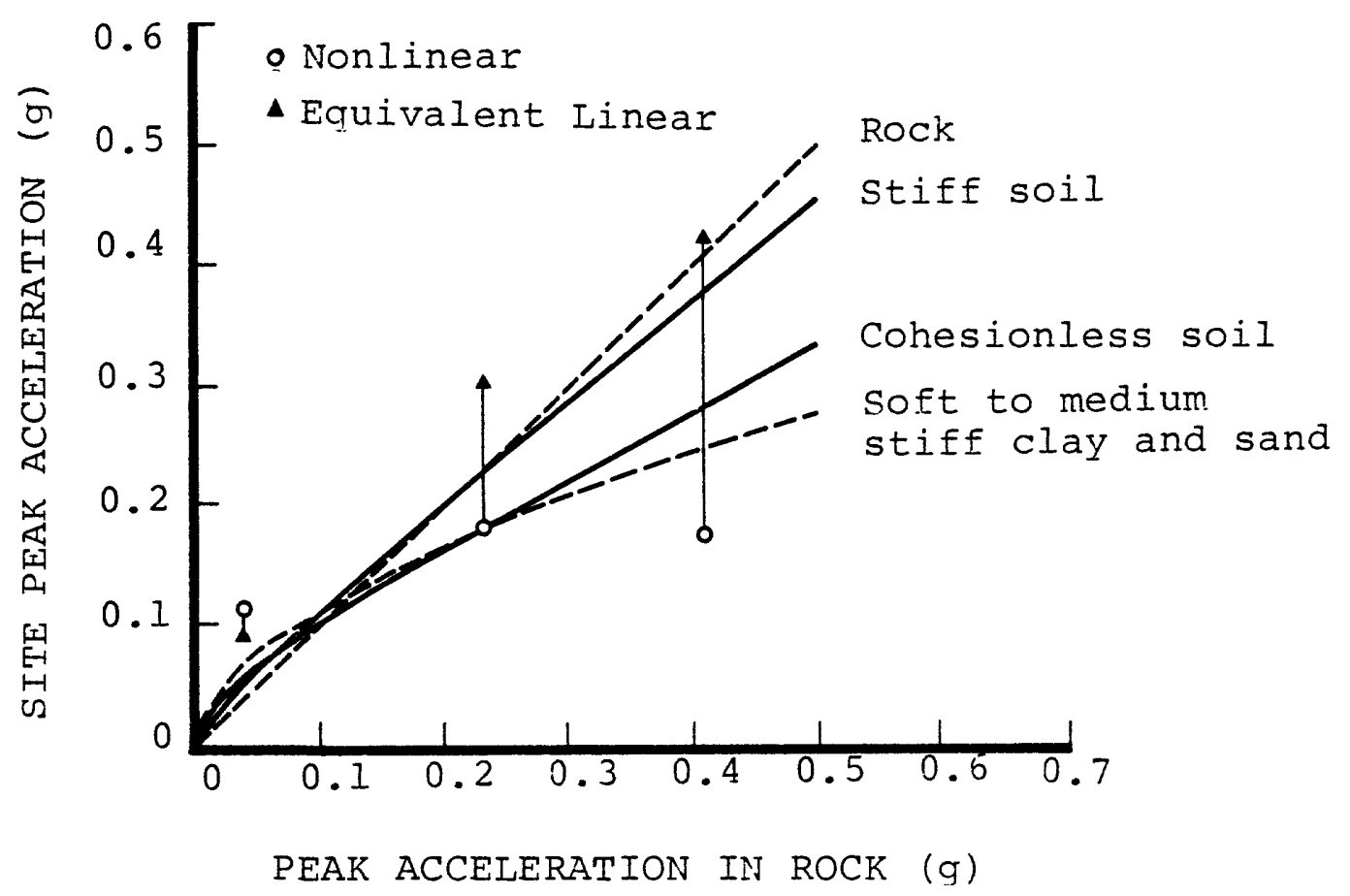

Figure 4.15. Relationship of peak soil site acceleration to peak bedrock acceleration for the soil site calculations of this study. The dashed and solid curves are from Seed, et al. (1976a), and represent approximate relationships for various site conditions derived from empirical distance attenuation curves (the empirical curves above $0.3 \mathrm{~g}$ are based on extrapolation of ground motion data). 
the bedrock value. The equivalent linear method is apparentiy incapable of adequately simulating the strong attenuative character of cohesionless soil under the high acceleration levels characteristic of the near-field.

The dependence of peak velocity data on site conditions appears to be more consistent than that of peak acceleration. Seed et. al. (1976a), Trifunac and Brady (1976), and Boore et. al. (1980) each find that peak velocity is systematically higher on soil sites than on rock sites. As Figure 4.14 shows, the computed velocity maxima are within the 70 percent prediction interval for the rock site calculations, and the least distant soil site calculation, and somewhat higher for the two more distant soil site calculations. Peak velocity is amplified by a factor of about 1.5 at 10 kilometers distance and 2.5 at 30 kilometers distance, which compares to 1.9 for the empirically derived (distance-independent) estimate of Trifunac (1976) for magnitude 5.5 events.

In summary, the nonlinear calculations are consistent with empirical trends governing peak motion where the trends are wellestablished by earthquake data. In the near-field, where bedrock acceleration peaks characteristically exceed 0.1 to $0.2 \mathrm{~g}$, nonlinear behavior is important in sharply reducing the value of peak soil acceleration, and the equivalent linear method did not reproduce this effect. Earthquake data are consistent with this sharp reduction in the near-field, but are sparse and inconclusive. Calculated peak velocities show amplification on soil sites comparable to that inferred empirically. The amplification effect disappears in the calculation at 5 kilometers distance, perhaps reflecting the predominance of high frequencies in the bedrock motion at this distance.

\subsubsection{Comparison of Nonlinear and Equivalent Linear Solutions}

In the last section we alluded to the inability of the equivalent linear approximation to model the sharp reduction of peak acceleration in the near field which was obtained for the nonlinear soil calculations. In this section we return to this issue and compare the nonlinear and equivalent linear results in more detail. 


\section{Time Histories}

Figures 4.15 and 4.17 compare time histories of free surface acceleration and velocity, respectively, computed by the two methods for Problems 1, 2, and 6 . The main differences in acceleration time histories are (1) the occurrence of large acceleration peaks in the equivalent linear calculations which are truncated in the nonlinear calculations, and (2) the fact that the equivalent linear method results in a smoother overall waveform, without the sharp cusps and short-period irregularities present in the nonlinear calculations.

The former effect probably reflects the finite shear strength inherent in the endochronic soil model. As discussed in Appendix B, the shear strength $H$ is a linear function of depth, and goes to zero at the surface. Therefore, the derivative of shear strength with respect to depth limits the shear stress gradient which can develop at the surface, and consequentiy limits the size of acceleration peaks. In our case, this upper limit would be about $0.37 \mathrm{~g}$; this upper limit was not actually realized in the calculations, however.

The second difference can be attributed to the fact that the equivalent linear method utilizes a time-independent damping which is determined by the peak strain, whereas the effective dampina provided by the nonlinear endochronic model is governed by the instantaneous strain amplitudes in the soil -- small amplitude disturbances incur little attenuation. Furthermore, nonlinear materials couple low- and high-frequency components of motion (see, for example, the analysis of valera et. al. 1978), a phenomenon which is absent from the equivalent linear calculations.

The velocity time histories obtained by the two methods, Figure 4.17, are in better accord. Here the predominant discrepancies in the equivalent linear solutions are (1) the characteristic period of the largest peak is too short, and (2) the predominant period of the late-time reverberations is too long. The first discrepancy again reflects the inability of the equivalent linear method to model the large damping occasioned by the shear 
EQUIVALENT LINEAR
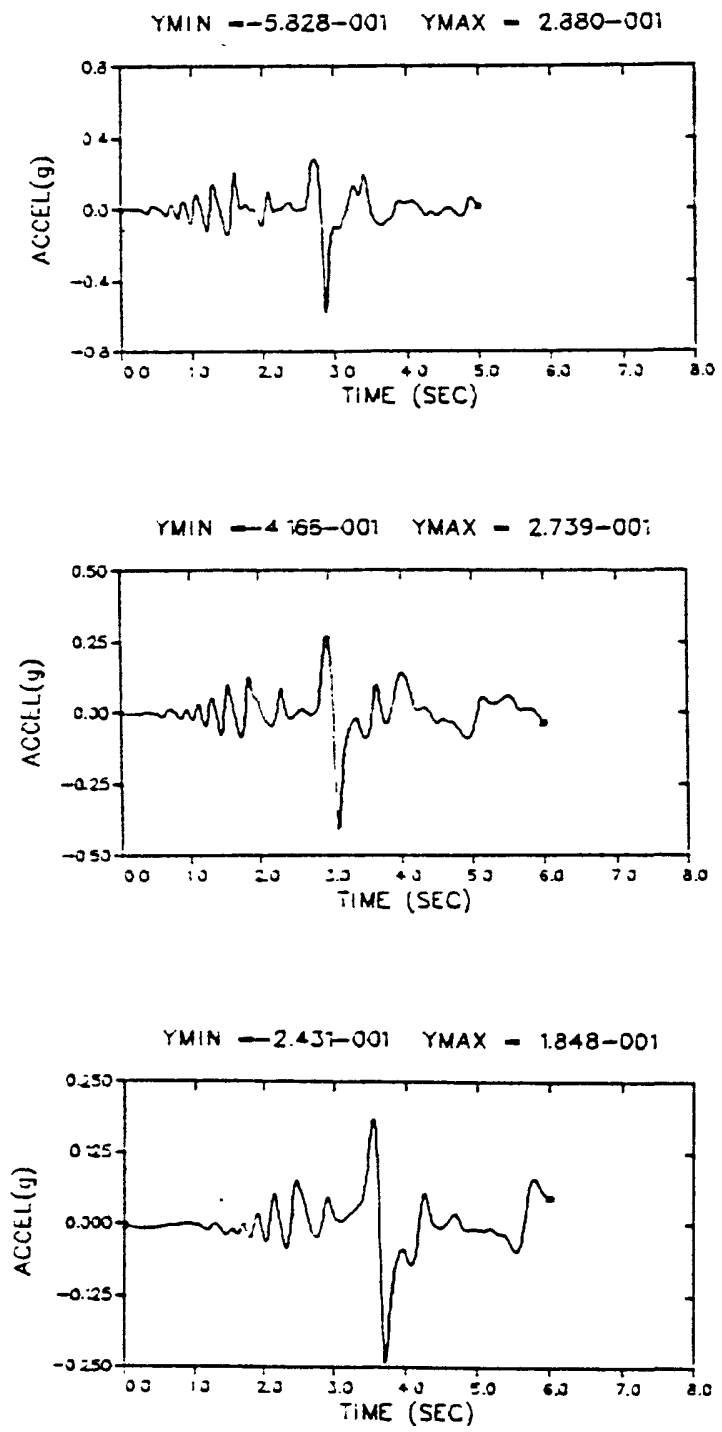

NONLINEAR
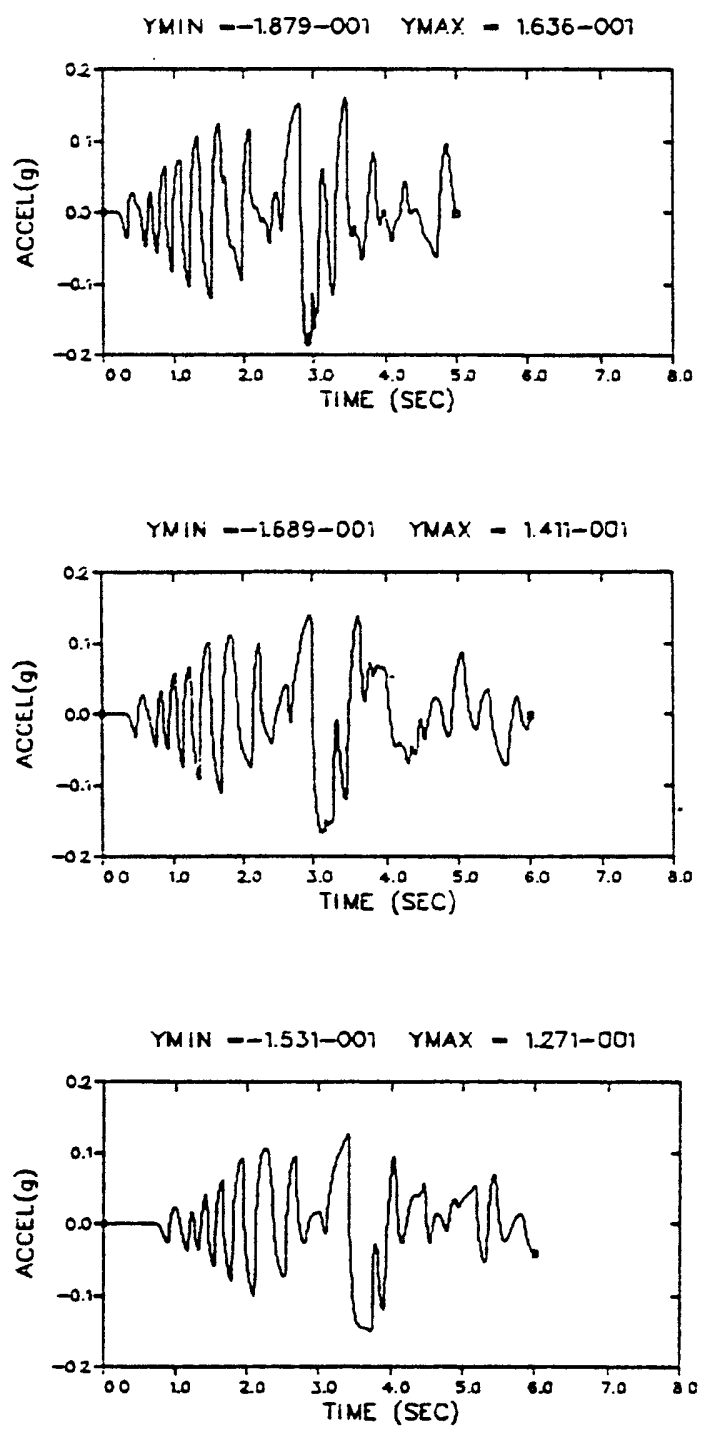

Figure 4.16. Comparison of acceleration time histories obtained by equivalent linear and nonlinear analyses, for Problems 1,2 , and 6 . 
EQUIVALENT LINEAR
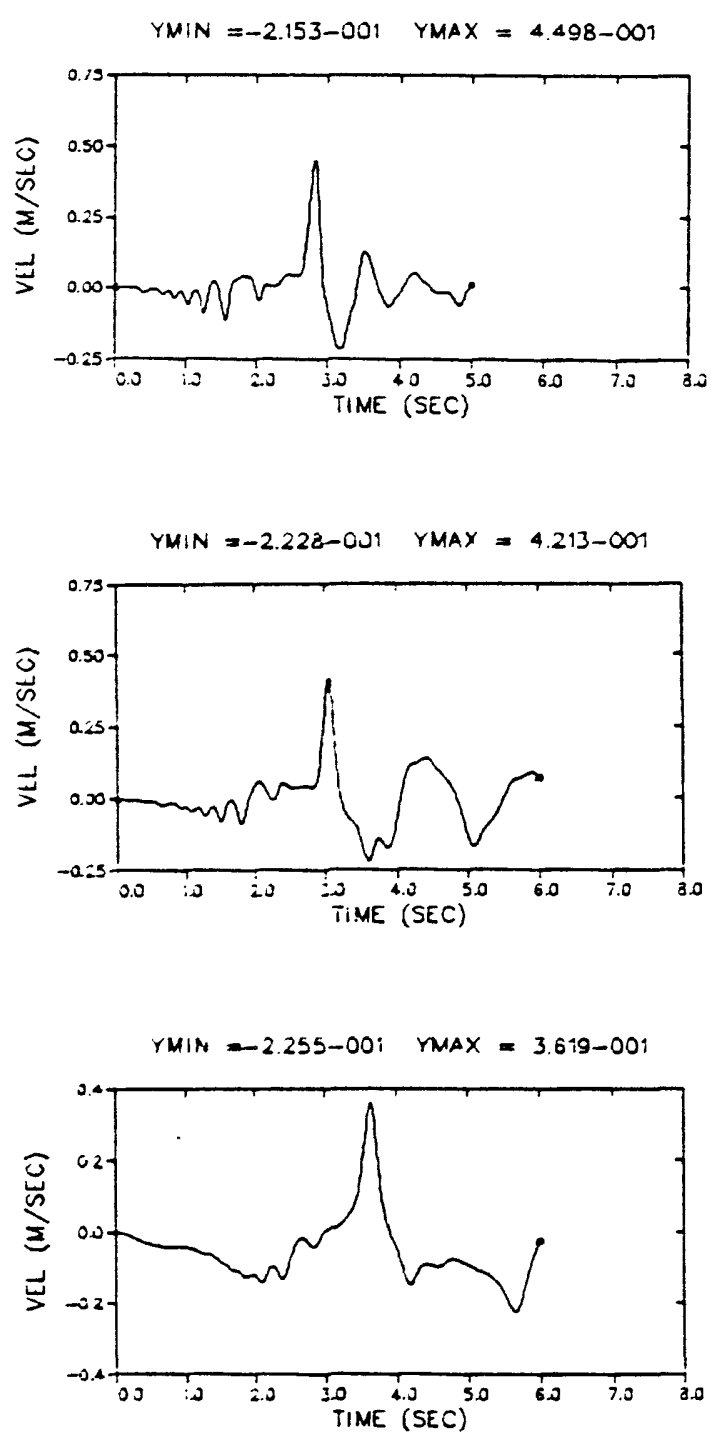

NONLINEAR
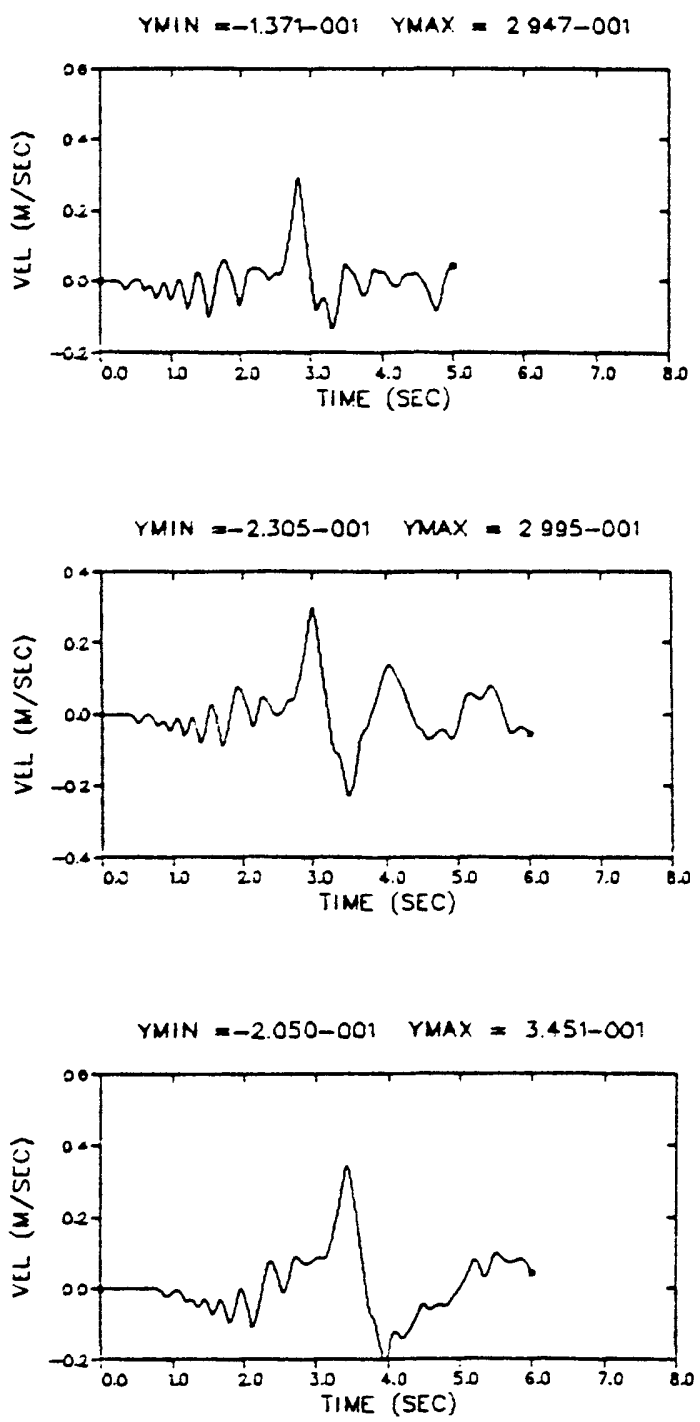

Figure 4.17. Comparison of velocity time histories obtained by equivalent linear and nonlinear analyses, for Problems 1,2 , and 6 . 
strength gradient in the soil. The second discrepancy is attributable to the fact that the equivalent linear shear modulus is determined by peak strain. The late-time reverberations, which involve strain amplitudes considerably lower than the peak strain, are controlled by an inappropriately low shear modulus, and therefore too low a resonant frequency.

\section{Peak Motion}

We have already noted the failure of the equivalent linear method to model the strong attenuation of large acceleration peaks which occurred in the nonlinear calculations. Figure 4.18 summarizes the comparison of peak accelerations and velocities predicted by each method. Peak acceleration and velocity are consistently overestimated by the equivalent linear method when the bedrock acceleration exceeds 0.1 to $0.2 \mathrm{~g}$. The overestimate of peak acceleration is greater than the overestimate of peak velocity.

Figures 4.19 and 4.20 present peak acceleration and velocity, respectively, as functions of depth, for three calculations at three different distances from the source. At the 30 kilometer site (bedrock peak acceleration equal to $0.04 \mathrm{~g}$ ), the two methods are in reasonable agreement throughout the soil column. Peak acceleration decreases with depth. At the two nearer sites there is generally very good agreement between the two methods at depths below about 5 meters; at about this depth, however, the equivalent linear method predicts an abrupt increase in peak motion, whereas the nonlinear method does not. At the two nearer sites, the equivalent linear method predicts that peak acceleration at depth is less than its surface value, while the nonlinear method predicts that peak acceleration at depth generally exceeds its surface value.

\section{Response Spectra}

Figure 4.21 shows response spectra (pseudo relative velocity) for two cases, Problem 2 and Problem 6. For Problem 6 (30 meter deep soil, 5 kilometers distance from the source) the equivalent 


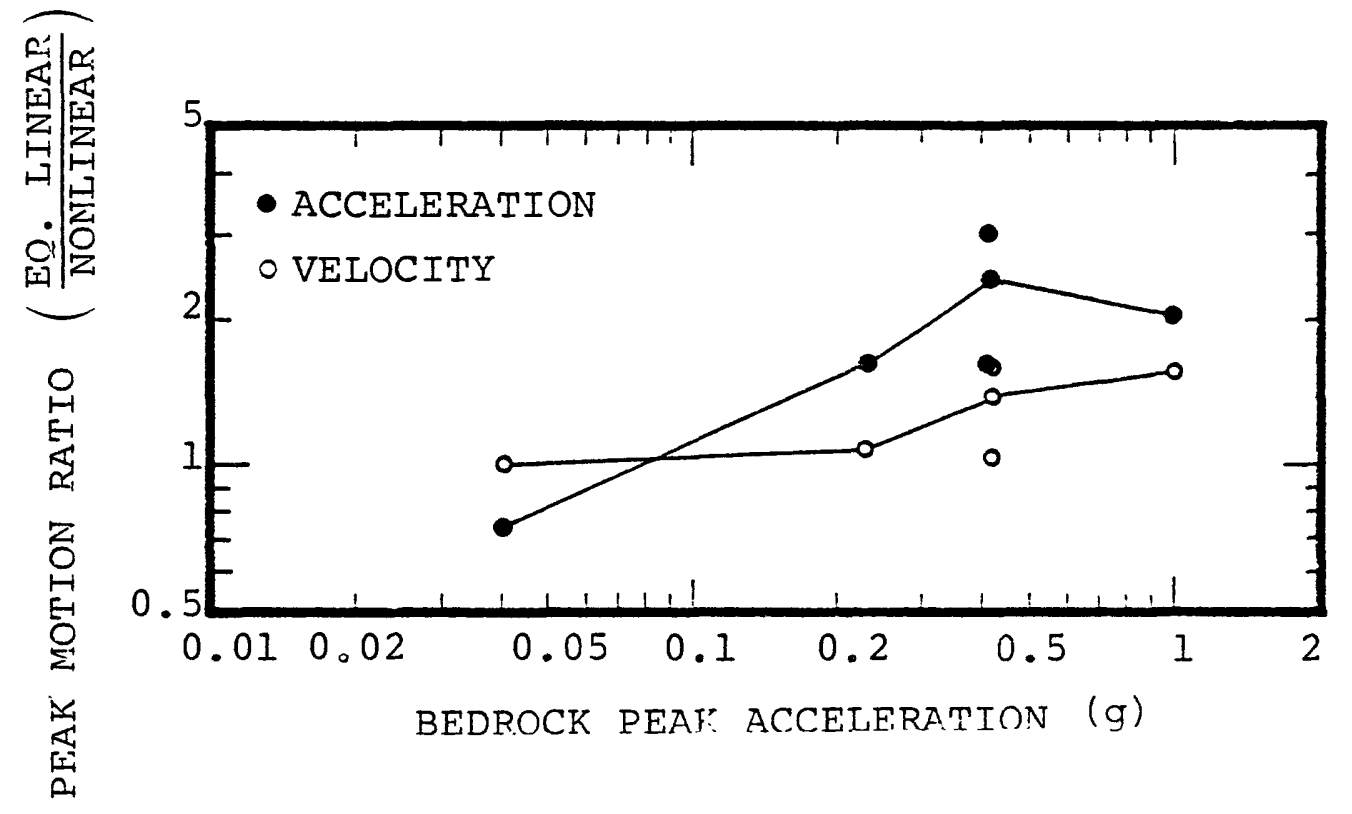

Figure 4.18. The ratio of peak motion obtained by the equivalent linear method to that obtained by nonlinear analys is, plotted versus the peak bedrock acceleration which was input. Lines connect points associated with calculations for $30 \mathrm{~m}$ soil layers. 


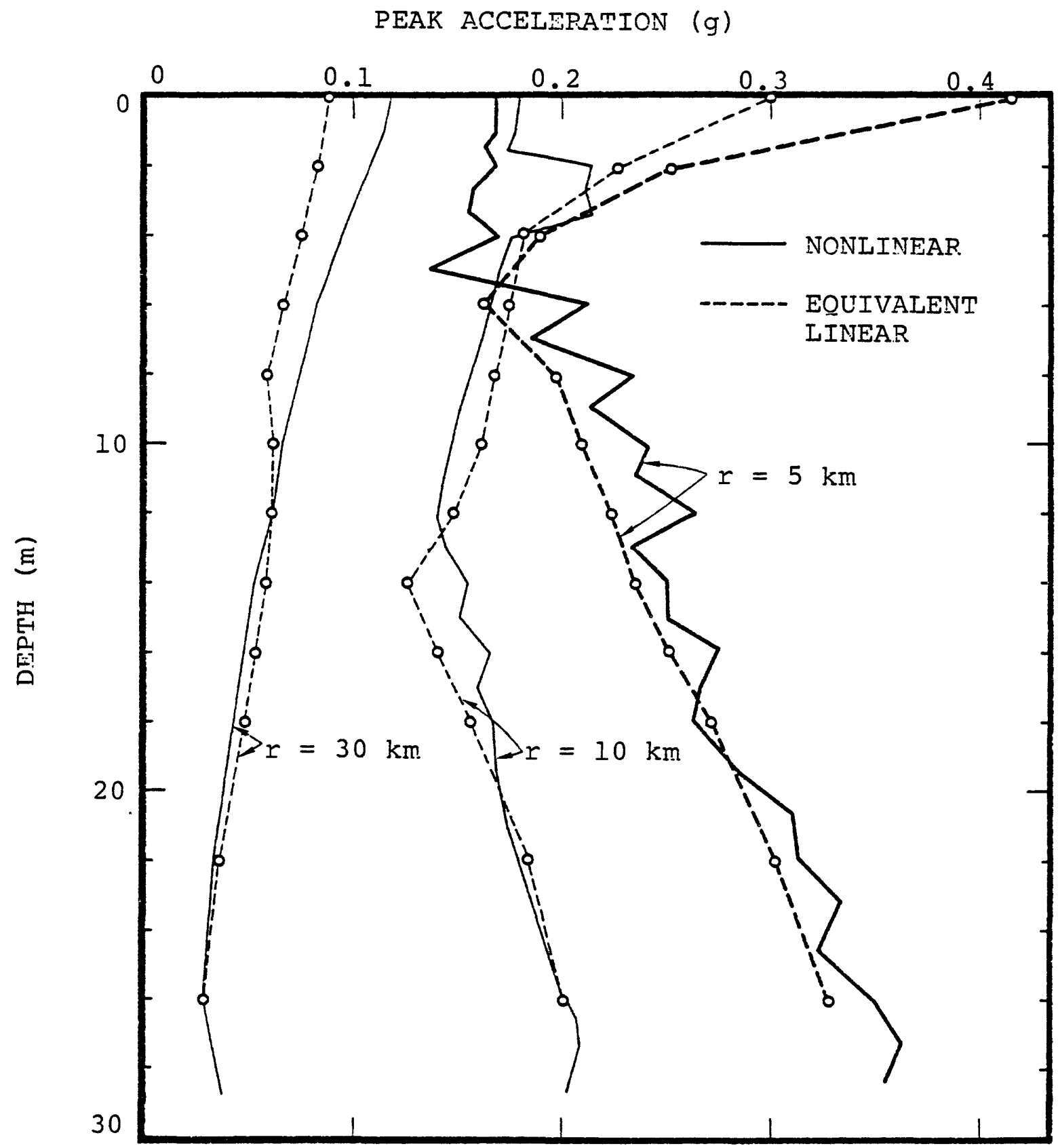

Figure 4.19. Peak acceleration versus depth, for nonlinear and equivalent linear calculations (Problems 6, 7, and 8). Open circles indicate depths at which output was obtained from the equivalent linear calculations. 
PEAT VELOCITY $(\mathrm{m} / \mathrm{sec})$

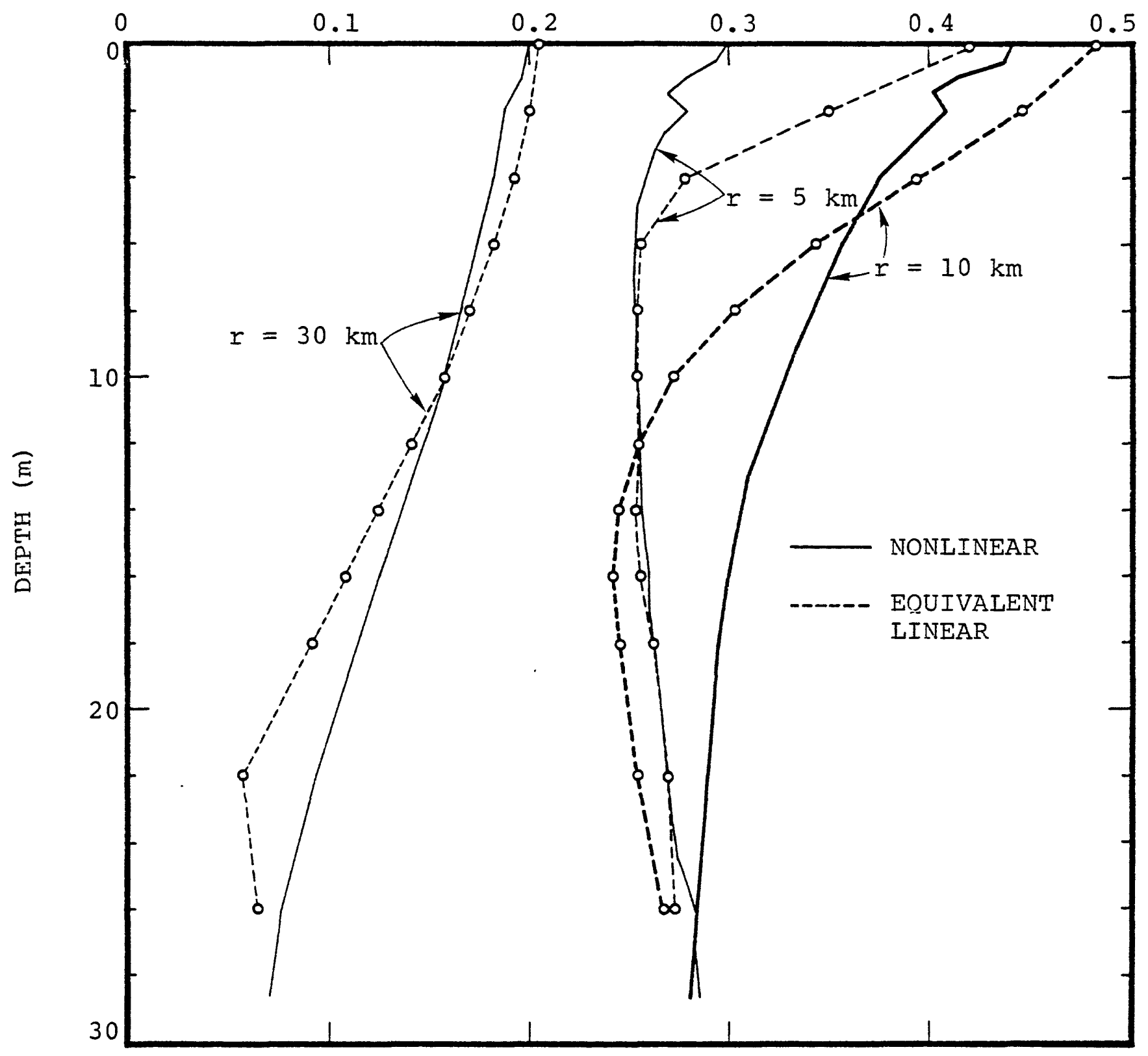

Figure 4.20. Peak velocity versus depth, for nonlinear and equivalent linear calculations (Problems 6, 7, and 8). Open circles indicate depths at which output was obtained from the equivalent linear calculations. 

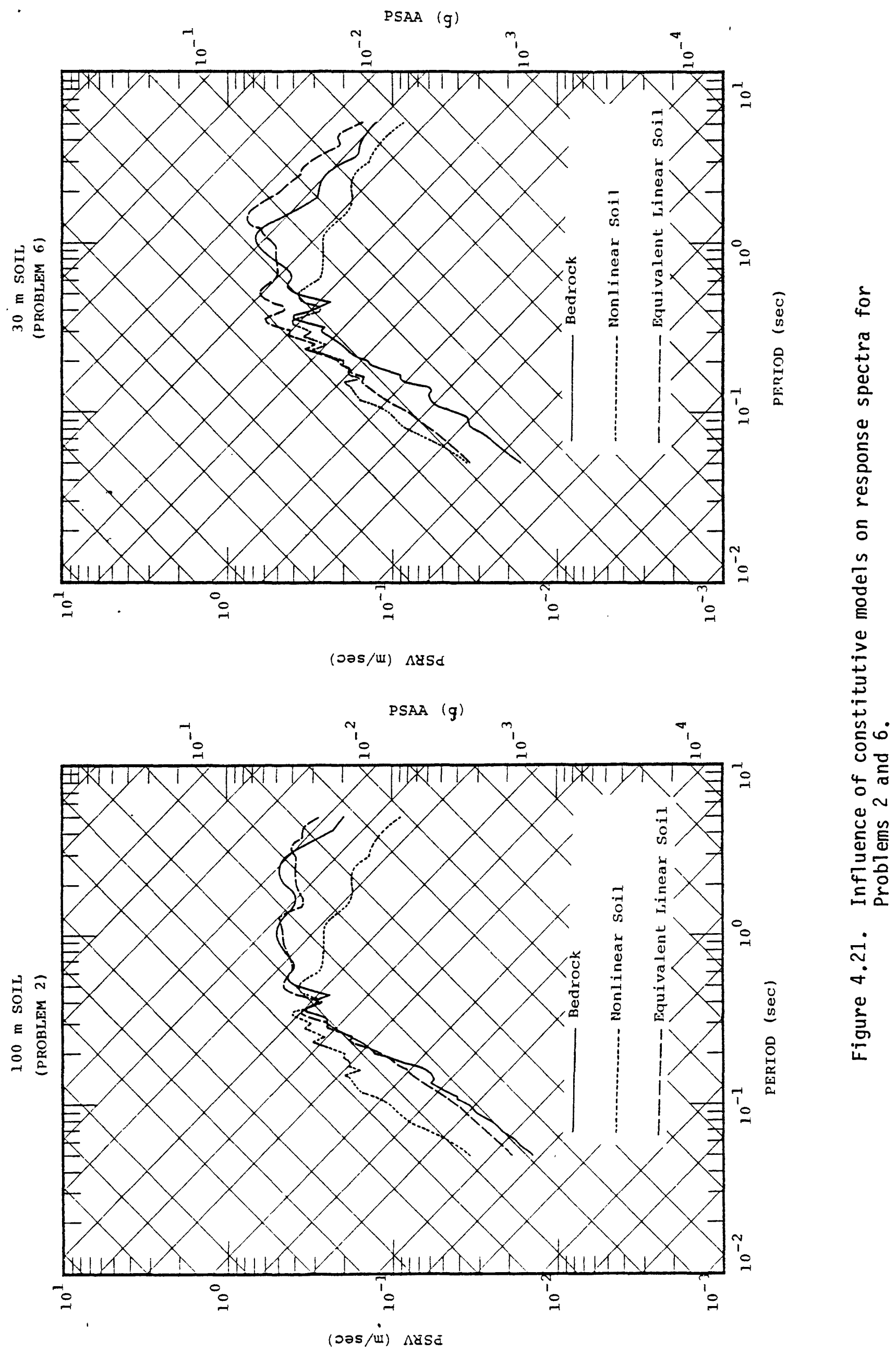
linear method overestimates the response over most of the spectrum, most conspicuously for periods below about 0.2 seconds. The spectral maximum at about one second period is shifted somewhat toward longer periods in the equivalent linear solution. For Problem 2 (100 meter deep soil, 5 kilometers distance) the two methods are in better overall agreement; the equivalent linear response exceeds the nonlinear response for periods below about 0.1 seconds.

Several previous investigations have compared nonlinear and equivalent linear response of soil columns to complex seismic input. Joyner and Chen (1975), Taylor and Larkin (1978), and Valera et al. (1978) each observe that the nonlinear response exceeds the equivalent linear response in the neighborhood of 0.1 to 0.5 second periods; this effect is not present in the response spectra of Figure 4.21. There is, however, a tendency for the nonlinear response to increase relative to the equivalent linear response in the 0.1 to 0.5 second range as the soil column thickness increases. This may reconcile our results with those of Joyner and Chen, who studied a soil column of 200 meters thickness. There is also a tendency for the nonlinear response to increase in this period range, relative to the equivalent linear response, when the input peak acceleration is reduced. This tendency is evidenced in Table 4.2, and perhaps reconciles our results with those of Taylor and Larkin, since their study employed an input time history with peak acceleration equal to only $0.14 \mathrm{~g}$. The results of valera et al. are somewhat more difficult to reconcile with ours, since they employed a 30.5 meter layer of dry sand and a maximum input acceleration of $0.5 \mathrm{~g}$, which is similar to our Problem 6 . They found that nonlinear response of one of their soil constitutive models exceeded the equivalent linear response in the 0.05 to 0.2 second period range, while the nonlinear response of the other constitutive model studied exceeded the equivalent linear response only in the 0.1 to $0 . ?$ second period range. Our sand constitutive 
TABLE 4.2

RATIO OF REPONSE SPECTRAL VALUES OBTAINED BY EOUIVALENT

LINEAR METHOD TO THOSE OBTAINED BY NONL INEAR METHOO

$$
\text { PSRV Ratio } \frac{\text { Equivalent Linear }}{\text { Nonlinear }}
$$

\begin{tabular}{|c|c|c|c|c|}
\hline $\begin{array}{l}\text { Problem } \\
\text { Number }\end{array}$ & $\begin{array}{c}\text { Peak Input } \\
\text { Acceleration }(\mathrm{g})\end{array}$ & $\begin{array}{l}0.1 \\
\mathrm{sec}\end{array}$ & $\begin{array}{l}0.2 \\
\text { sec }\end{array}$ & $\begin{array}{l}0.5 \\
\text { sec }\end{array}$ \\
\hline 3 & 1.0 & 1.33 & 1.11 & 1.60 \\
\hline 6 & 0.41 & 1.92 & 1.49 & 1.63 \\
\hline 7 & 0.23 & 1.13 & 1.37 & 1.42 \\
\hline 8 & 0.04 & 0.82 & 0.92 & 1.03 \\
\hline
\end{tabular}


model differs from the two used by valera et. al., and we have used model parameters corresponding to an artificially loose sand (void ratio 0.8 , compared to 0.5 for valera et. al.), so it is not unreasonable that our nonlinear response is lower relative to the equivalent linear analys is in this restricted period range.

In summary, we find that the performance of the equivalent linear approximation for the response of cohesionless soil depends on both the nature of the soil deposit and the amplitude level of the base rock acceleration. Peak motion estimates by the equivalent linear method are adequate for peak input accelerations less than 0.1 to $0.2 \mathrm{~g}$, but are excessively conservative for stronger input accelerations. The overestimate of peak motion by the equivalent linear method is apparently due to an inability of the method to adequately model energy loss in the uppermost several meters of the soil column. Response spectra for the equivalent linear calculations are generally too conservative in the short period $(0.1$ to 0.5 second) range when the input acceleration is large. This result contrasts with that of other recent investigations, but the discrepancy is not great and appears to be associated with the combined effects of soil thickness, input acceleration level, and nature of the soil representation. 


\section{SUMMARY OF RESULTS}

In this investigation, our objective has been to develop and evaluate a new analytical approach to estimating site-dependent earthquake ground motion. The approach is based on (1) assuming a physical model for the earthquake source and wave propagation path to the site, then using theoretical seismogram methods to compute base-rock seismic motion at the site, (2) applying a new endochronic constitutive model to represent the hysteretic response of local soil deposits, and (3) computing surface motion at the site using a transient, dynamic finite element method. The predictive capabilities of the approach were examined by comparing ground motion calculations with (1) empirically derived ground motion trends and (2) ground motion simulated by the equivalent linear method. The analytical approach has been structured to potentially provide a three-dimensional ground motion capability, in that the theoretical seismic method, the endochronic constitutive theory, and the transient finite element method each have been developed within a fully three-dimensional framework.

In Section 2, we described the construction of base-rock ground motion for a simplified earthquake source model. The earthquake model consisted of an assemblage of discrete, circular cracks, each described by the Sato and Hirasawa approximate solution, representing an extended source. Propagation of the source disturbance through a stratified, anelastic earth model was accomplished by a direct wavenumber integration method, with calculations restricted to the SH component of motion. The Parkfield earthquake was used as a guide to selection of source parameters. Peak acceleration, peak velocity, and strong motion duration were required to correlate with near-field recordings of that event in the distance range 5 to $10 \mathrm{~km}$, and once the source parameters were adjusted to give this agreement, it was found that reasonable agreement was automatically achieved with empirical ground motion trends at $30 \mathrm{~km}$ distance. The study led to the following conclusions: 
- Strong motion observations are, in general, inconsistent with a rupture mechanism in which crack growth stops instantaneousiy. More realistic rupture deceleration is required in the simulations to achieve acceptable high frequency ground motion estimates in the near field.

- The SH component of motion is apparently adequate for simulating ground motion consistent with observed values of peak acceleration and velocity. Provided some source complexity is introduced, as in our composite model, reasonable strong motion duration can also be simulated with the $S H$ component alone. However, neglect of the P-SV component results in somewhat unrealistic time histories; in particular, rupture through an $\mathrm{SH}$ radiation-pattern node produces an artificial "quiet" period in the accelerograms. Addition of the P-SV contribution would substantially improve the qualitative agreement with observed ground motion time histories, but would probably have little effect on peak motion estimates.

- Similarity of the source wavelets from each individual source event, as well as the uniformity of the assumed rupture velocity, lead to artificial periodicities in the simulated ground motion. Introduction of variable rupture velocity and variable source dimensions would give more realistic waveforms.

Section 3 described a new constitutive model for soils, the Endochronic model. The model was fit to cyclic shear data for dry sand, and then an equivalent linear representation was derived for the same sand data. We note the following points: 
- In agreement with observed behavior of soils, the new endochronic model predicts a gradual accumulation of plastic strain from the onset of loading. Furthermore, it exhibits elastic behavior at the initiation of unloading and reloading processes, and closure of all hysteresic loops. The general three-dimensional version of the model also has the capability to describe most of the features of soil behavior important for seismic loading, including densification, dilatancy, strain hardening/softening, and cyclic creep (ratcheting).

- Using the endochronic model, close agreement was achieved between calculated and observed response of dry sand over 300 cycles of deformation.

- Only five parameters were required to accomplish the fit to the sand data.

The constitutive models of Section 3 and the base-rock ground motion simulations of Section 2 were applied in Section 4 to compute soil site ground motion. A transient finite element method was used to compute the response of a column of dry sand whose constitutive behavior was represented by the endochronic model; the SHAKE code was used to compute the response of the same soil column represented by the equivalent linear model. The main conclusions from this part of the study are given below.

- The endochronic constitutive model performs very effectively in a wave propagation code under complex loading histories such as those provided by our synthetic seismograms. In particular, the calculations clearly illustrate the following features: (i) loading and unloading initiate along the elastic slope, 
(ii) all hysteresis loops close, (ii i) the model does not display the unrealistic "corners" displayed by Iwan-type models when a loading or unloading curve interesects a previous loading, or unloading, curve, (iv) significant strain hardening occurs through the deformation history, and ( $v$ ) ratcheting develops under asymmetric loading.

- The ground motion calculations with the Endochronic model are not strongly sensitive to details of the discretization of the soil, even though the distribution of maximum shear strain may be.

The nonlinear calculations are consistent with empirical trends governing peak motion where the trends are well-established by earthquake data. In the near-field, where bedrock acceleration peaks characteristically exceed 0.1 to $0.2 \mathrm{~g}$, nonlinear behavior is important in sharply reducing the value of peak soil acceleration, and the equivalent linear method did not reproduce this effect. Earthquake data are consistent with this sharp reduction in the near-field, but are sparse and inconclusive. Calculated peak velocities show amplification on soil sites comparable to that inferred empirically. The amplification effect disappears in the calculation at 5 kilometers distance, perhaps reflecting the predominance of high frequencies in our modeling of bedrock motion at this distance.

The performance of the equivalent linear model for the response of cohesionless soil depends on both the nature of the soil deposit and the 
amplitude level of the baserock acceleration. Peak motion estimates by the equivalent linear method are adequate for peak input accelerations less than 0.1 to $0.2 \mathrm{~g}$, but are excessively conservative for stronger input accelerations. The overestimate of peak motion by the equivalent linear method is apparently due to an inability of the method to adequately model energy loss in the uppermost several meters of the soil column. Response spectra obtained from the equivalent linear calculations are generally too conservative in the short period ( 0.1 to 0.5 second) range when the input acceleration is large. This result contrasts with that of other recent investigations, but the discrepancy is not great and appears to be associated with the combined effects of soil thickness, input acceleration level, and nature of the soil representation. 


\section{REFERENCES}

Aki, K., 1979, "Characterization of Barriers on an Earthquake Fault," J. Geophys. Res.,, Vol. 84, op. 6140-6148.

Aki, K., and M. Bouchon, 1977, "Discrete Wavenumber Representation of Seismic Source Wave Fields," Bull. Seism. Soc. Amer., Vol. 67, pp. 259-278.

Aki, K. and P. G. Richards, 1980, Oualitative Seismology, Theory and Methods, W. H. Freeman and Company, San Francisco.

Anderson, J. G., 1974 "A Dislocation Model for the Parkfield Earthquake," Bu11. Seism. Soc. Amer., Vo1. 64, pp 671-686.

Andrews, D. J., 1979, "A Stochastic Fault Model," Preprint.

Apse1, R. J., 1979, "Dynamic Green's Functions for Layered Media and Applications to Boundary-Value Problems," Ph.D. dissertation, University of California, San Diego.

Archuleta, R. J., and S. M. Day, 1980, "Dynamic Rupture in a Layered Medium: An Example, the 1965 Parkfield Earthquake," Bul1. Seism. Soc. Amer., Vol. 70, (in press)

Bache, T. C., and T. G. Barker, 1978, "The San Fernando Earthquake A Model Consistent with Near-Field and Far-Field Observations at Long and Short Periods," Systems, Science and Software Final Technical Report submitted to the U. S. Geological Survey, SSS-R-79-3552.

Bache; T. C., D. G. Lambert and T. G. Barker, 1980, "A Source Model for the March 28, 1975 Pocatello Valley Earthquake from Time-Domain Modeling of Teleseismic P Waves," Bull. Seism. Soc. Amer., Vol. 70, pp 405-418.

Bazant, Z. P., 1974, "A New Approach to Inelasticity and Failure of Concrete, Sand and Rock: Endochronic Theory," Proc. 11th Annual Mtg., Soc. Engr. Science, G. J. Dvorak, Editor, Duke University, Durham, North Carolina, p. 158.

Bazant, Z. P., and R. J. Krizek, 1976, "Endochronic Constitutive Law for Liquefaction of Sand," J. Engr. Mechs. Div., ASCE, Vol. 102, EM2, Apri1 1976, p. 225.

Bazant, Z. P., R. J. Krizek and C. -L. Shieh, 1979, "Hysteretic Endochronic Theory for Sand," Northwestern University, Evanston, Illinois, Report 79-4/654h, April. 
Ben-Menahem, A., and S. J. Singh, 1968, "Multipolar Elastic Fields in a Layered Halfspace," Bull. Seism. Soc. Amer., Vol. 58, pp. $1519-1572$.

Bieber, R. E., and H. J. Hovland, 1980, "Seismic Dynamic Response by Approximate Methods," Earthquake Engineering and Structural Dynamics, Vol. 8,1980, p. 41 .

Boatwright, J., 1980, "A Spectral Theory for Circular Seismic Sources; Simple Estimates of Source Dimension, Dynamic Stress Drop, and Radiated Seismic Energy," Bull. Seism. Soc. Amer., Vol. 70, pp. 1-28.

Boore, D. M., 1972, "Finite Difference Methods for Seismic Wave Propagation in Heterogeneous Materials," in Methods in Computational Physics, Vol. 11, (B. A. Bolt, Ed.) Academic Press, New York.

Boore, D. M., and W. B. Joyner, 1978, "The Influence of Rupture Incoherence on Seismic Directivity," Bull. Seism. Soc. Amer., Vol. 68 , pp. 283-300.

Boore, D. M., W. B. Joyner, A. A. 0liver, and R. A. Page, 1980, "Peak Acceleration, Velocity, and Displacement from Strong-Motion Records," Bull. Seism. Soc. Amer., Vol. 70, February, p. 305.

Bouchon, M., 1978, "A Dynamic Source Model for the San Fernando Earthquake," Bull. Seism. Soc. Amer., Vol. 68, pp. 1555-1576.

Bouchon, M., 1979, "Predictability of Ground Displacement and Velocity Near an Earthquake Fault: An Example: The Parkfield Earthquake of 1966," J. Geophys. Res., Vol. 84, pp. 6149-6156.

Chapman, C. H., 1978, "A New Method for Computing Seismograms," Geophys. J., Vol. 54, pp. 481-518.

Cuellar, V., Z. P. Bazant, R. J. Krizek and M. L. Silver, 1977, "Densification and Hysteresis of Sand under Cyclic Shear," J. Geot. Engr. Div., ASCE, GT5, May 1977, p. 399.

Dahlen, F. A., 1974, "On the Ratio of P-Wave to S-Wave Corner Frequencies for Shallow Earthquake Sources," Bull. Seism. Soc. Amer., Vol. 64, pp. 1159-1180.

Dahlquist, G. and Bjorck, A., 1974, Numerical Methods, Prentice-Hal1, Englewood Cliffs, New Jersey.

Day, S. M., 1979, "Three-Dimensional finite Difference Simulation of Fault Dynamics," Systems, Science and Software Final Report sponsored by the National Aeronautics and Space Administration. SSS-R-80-4295. 
Del Mar Technical Associates (DELTA),1978, "Simulation of Earthquake Ground Motions for San Onofre Nuclear Generating Station Unit 1," Final Report for Southern California Edison Company, submitted for review to the Nuclear Regulatory Commission.

Del Mar Technical Associates (DELTA),1979, "Simulation of Earthquake Ground Motions for San Onofre Nuclear Generating Station Unit 1: Supplement 1," supplemental report submittd to Southern California Edison Company, July.

Donovan, N. C., 1973, "A Statistical Evaluation of Strong Motion Data Including the February 9, 1971, San Fernando Earthquake," Proc. Fifth World Conf. Earthquake Eng., Rome, Vol. 1, pp. 1252-1261.

Drnevich, V.P., J. R. Hall, Jr., and F. E. Richart, Jr., 1967, "Effects of Amplitude of Vibration on the Shear Modulus of Sand," Proc. Int1. Symp. on Wave Prop. and Dyn. Properties of Earth MatTs., University of New Mexico, Albuquerque, New Mexico.

Duffy, J., and R. D. Mindlin, 1957, "Stress-Strain Relations and Vibrations of a Granular Medium," J. Appl. Mechs., Vol. 24, p. 585.

Duke, C. M., K. E. Johnsen, L. E. Larson, and D. C. Engman, 1972, "Effects of Site Classification and Distance on Instrumental Indices in the San Fernando Earthquake," University of California, Los Angeles, School of Engineering and Applied Science, UCLA-ENG-7247, p. 29.

Faccioli, E. E., V. Santayo, and J. L. Leon, 1973, "Microzonation Criteria and Seismic Response Studies for the City of - Managua," Proc. Earthquake Eng. Res. Dist. Conf. Managua, Nicaragua, Earthquake of December 23, 1972 I, pp. 271-291.

Finn, W. D. Liam, Kwok Wing Lee and G. R. Martin, 1975, "Stress Strain Relations for Sand in Simple Shear," Presented at "Seismic Problems in Geotechnical Engineering," ASCE Meeting, Denver, Colorado, November.

Frazier, G. A., and Petersen, C. M., 1974, "3-D Stress Waves Code for the ILLIAC IV," Systems, Science and Software Report SSS-R-74-2103.

Fung, Y. C., 1965, Foundation of Solid Mechanics, Prentice-Hall, Englewood Cliffs, New Jersey.

Hanks, T. C., 1974, "The Faulting Mechanisms at the San Fernando Earthquake," J. Geophys. Res.,, Vol. 79, pp 1215-1229. 
Hanks, T. C., 1979, "Six Values and $\omega^{-\gamma}$ Seismic Source Models: Implications for Tectonic Stress Variations Along Active Crustal Fault Zones and the Estimation of High Frequency Strong Ground Motion," J. Geophys. Res.,, vol. 84, pp 2235-2242.

Hardin, B. 0., and F. E. Richart, Jr., 1963, "Elastic Wave Velocities in Granular Soils," J. Soil Mechs. Founds, Div., ASCE, Vol. 89, Proc. Paper 3407, February, p. 33.

Hardin, B. 0., and V. P. Drnevich, 1972, "Shear Modulus and Damping in Soils: Measurement and Parameter Effects," J. Soil Mechs. Founds. Div., ASCE, Vol. 98, p. 603.

Hartze11, S. H. and J. N. Brune, 1979, "The Horse Canyon Earthquake of August 2, 1975 - Two-Stage Stress-Release Process in a Strike Slip Earthquake," Bu11. Seism. Soc. Amer., Vol. 59, pp. 1161-1174.

Haske11, N. A. , 1953, "The Dispersion of Surface Waves in Multilayered Media, Bul1. Seism. Soc. Amer., Vol 43, p. 17.

Heaton, T. H., and D. V. Helmberger, 1979, "Generalized Ray Models of the San Fernando Earthquake," Bull. Seism. Soc. Amer., Vol. 69, pp. 1311-1135.

Herrmann, R. B. , 1978, "A Note on Causality Problems in the Numerical Solution of Elastic Wave Propagation in Cylindrical Coordinate Systems," Bu11. Seism. Soc. Amer., Vol. 68, pp. 117-123.

Housner, G. W., and P. C. Jennings, 1964, "Generation of Artificial Earthquakes," J. Eng. Mech. Div. ASCE, Vol. 90, pp 113-150.

Idriss, I. M., and H. B. Seed, 1968, "Seismic Response of Horizontal Soil Layers," J. Soil Mechs. Founds. Div., ASCE, Vol. 94, SM4, July, p. 1003.

Isenberg, J., D. K. Vaughn and I. S. Sandler, 1978, "Nonlinear Soil-Structure Interation," Weidlinger Assocs., Menlo Park, California, Report EPRI NP-945, December.

Joyner, W. B., and A. T. F. Chen, 1975, "Calculation of Nonlinear Ground Response in Earthquakes," Bull. Seism. Soc. Amer., Vol. 65 , No. 5, p. 1315.

Kanamori, H., 1979, "A Semi-Empirical Approach to Prediction of Long-Period Ground Motions from Great Earthquakes," Bu11. Seism. Soc. Amer., Vol. 69., pp. 1645-1670.

Kjartansson, E., 1979, "Constant O-Wave Propagation and Attenuation," J. Geophys. Res.,, Vol. 84, pp. 4737-4748. 
Konder, R. L., 1963, "Hyperbolic Stress-Strain Response: Cohesive Soils," J. Soil Mechs. Founds. Div., ASCE, Vol. 89, SM1, Proc. Paper 3429, p. 115.

Kostrov, B. V., 1964, "Self-Similar Problems of Propagating Shear Cracks," J. App1. Math Mech., Vo1. 28, pp 1077-1087.

Lindh, A. G. and D. M. Boore, 1973, "Another Look at the Parkfield Earthquake Using Strong-Motion Instruments as a Seismic Array," (abstract) presented at the 68th Annual National Meeting of the Seismological Society of America.

Lysmer, J., H. B. Seed, and P. Schnabel, 1971, "Influence of Base-Rock Characteristics on Ground Response," Bull. Seism. Soc. Amer., , Vol. 61, p. 1213.

Madariaga, R., 1976, "Dynamics of an Expanding Circular Fault," Bul1. Seism. Soc. Amer., Vol. 66, pp. 639-666.

McGarr, A., S. M. Spottiswoode, N. C. Gay and W. D. Ortlepp, 1979, "Observations Relevant to Seismic Driving Stress, Stress Drop, and Efficiency," J. Geophys. Res.,, vol. 84, pp 2251-2261.

Mohraz, B. 1976, "A Study of Earthquake Response Spectra for Different Geological Conditions," Bull. Seism. Soc. Amer., Vol. 66 , p. 915.

Newmark, N. M., A. R. Robinson, A. H.-S. Ang, L. A. Lopez and W. J. Hall, 1972, "Methods for Determining Site Characteristics," Proc. Int1. Microzonation Conference, Seattle, Washington, p. 113.

Page, R. A., D. M. Boore, W. B. loyner and H. W. Coulter, 1972, "Ground Motion Values for Use in the Seismic Design of the Trans-Alaska Pipeline System," U.S. Geological Survey Circular 672.

Pande, G. N., and 0. C. Zienkiewicz, 1980, Eds., Proc. Int1. Symp. on Soils Under Cyclic and Transient Loading, Swansea, WaTes, January 1980. A. A. Balkema, publisher.

Pyke, R., 1979, "Nonlinear Soil Models for Irregular Cyclic Loadings," J. Geot. Engr. Div., ASCE, Vol. 105, June, p. 715.

Read, H. E., and K. C. Valanis, 1979, "An Endochronic Constitutive Model for General Hysteretic Response of Soils," Final Report to EPRI, Systems, Science and Software, La Jolla, California, Report NP-957, January.

Richart, F. E., 1975, "Some Effects of Dynamic Soil Properties on Soil-Structure Interaction," Journal of the Geotechnical Engineering Division, ASCE, Vol. 101, p. 1197. 
Sandler, I. S., 1978, "On the Uniqueness and Stability of Endochronic Theories of Material Behavior," J. Appl. Mechs., vo 1. 45, p. 263.

Sato, T., and T. Hirasawa, 1973, "Body Wave Spectra from Propagating Shear Cracks," J. Phys. Earth, Vo1. 21, pp 415-431.

Schnabel, P. B., Lysmer, J., and Seed, H. B., 1972, "SHAKE -- A Computer Program for Earthquake Response Analysis of Horizontally Layered Sites," Earthquake Engineering Research Center, University of California, Berkeley, Report No. EERC 72-12, December.

Schnabel, P., H. B. Seed and J. Lysmer, 1972, "Modification of Seismograph Records for Effects of Local Soil Conditions," Bul1. Seism. Soc. Amer., Vol. 62, p. 1649.

Seed, H. B., and P. B. Schnabel, 1972, "Soil and Geologic Effects on Site Response During Earthquakes," Proc. Intl. Microzonation Conference, Seattle, Washington, Vol. 61.

Seed, H. B., R. Murarka, J. Lysmer, and I. M. Idriss, 1975a, "Relationships of Maximum Acceleration, Maximum Velocity, Distance from Source, and Local Site Conditions for Moderately Strong Earthquakes," Bu11. Seism. Soc. Am., Vol. 66, pp. 1323-1342.

Seed, H. B., C. Ugas, and J. Lysmer, 1976b, "Site-Dependent Spectra for Earthquake-Resistant Design," Bull. Seism. Soc. Amer., Vol. $56, p .221-243$.

Silver, M. L., and H. B. Seed, 1971, "Deformation Characteristics of Sands Under Cyclic Loading," J. Soil Mechs. Founds. Miv., ASCE, Vol. 97, SM9, p. 1171.

Streeter, V. L., E. B. Wylie and F. E. Richart, Jr., 1974, "Soil Motion Computations by Characteristics Method," J. Geot. Engr. Div., ASCE, Vol. 100, No. GT3, p. 247.

Swanger, H. J. and D. M. Boore, 1978, "Importance of Surface Waves in Strong Ground Motion in the Period Range of 1 to 10 Seconds," Proceedings of the Second International Conference on Microzonation, San Francisco, California, pp 1147-1457.

Taylor, P. W., and T. J. Larkin, 1978, "Seismic Site Response of Nonlinear Soil Media," J. Geot. Engr. Div., ASCE, Vol. 104, GT3, March, p. 369.

Trifunac, M. D. and A. G. Brady, 1976, "Correlations of Peak Acceleration, Velocity, and Displacement with Earthquake Magnitude, Distance, and Site Conditions," Earthquake Eng. Structural Dyn., Vol. 4, pp. 355-471. 
Trifunac, M. D., 1976, "Preliminary Analysis of the Peaks of Strong Earthquake Ground Motion - Dependence of Peaks on Earthquake Magnitude, Epicentral Distance, and Recording Site Conditions," Bull. Seism. Soc. Am., Vol. 60, pp. 137-160.

Valanis, K. C. , 1971a, "A Theory of Plasticity Without a Yield Surface, Part I - General Theory," Arch. Mekan. Stos., Vol. 23 , p. 517 .

Valanis, K.C., 1971b, "A Theory of Plasticity Without a Yield Surface, Part II - Application to the Mechanical Behavior of Metals," Arch. Mekan. Stos., Vol. 23, p. 535.

Valanis, K. C., 1974, "Effect of Prior Deformation on Cyclic Response of Metals," J. App1. Mechs., Vo1. 41, p. 441.

Valanis, K. C., 1975, "An Energy Probability Theory of Fracture (An Endochronic Theory)," J. de Mecanique, Vol. 14, p. 343.

Valanis, K. C., 1979, "Endochronic Theory with Proper Hysteresis Loop Closure Properties," Systems, Science and Software, La Jol1a, California, Report No. SSS-R-80-4182, August.

Valanis, K. E., 1980, "Endochronic Theory Numerical Scheme," private communication to H. E. Read, April.

Valanis, K. C., and H. -C. Wu, 1975, "Endochronic Representation of Cyclic Creep and Relaxation of Metals," J. Appl. Mechs., Vol. 42 , p. 67.

Valanis, K. C., and H. E. Read, 1978, "A Theory of Plasticity for Hysteretic Materials. Part I: Shear Response," J. Computers Structures, Vol. 8 , p. 503.

Valanis, K. C., and H. E. Read, 1979, "A New Endochronic Plasticity Model for Soils," Interim Report to EPRI, Systems, Science and Software, La Jolla, California, Report No. SSS-R-80-4294, December.

Valanis, K. C., and H. E. Read, 1980, "Recent Development and Applications of the Endochronic Theory to the Behavior of Soils," presented at the Int1. Symp. on Soils under Cyclic and Transient Loading, Swansea, Wales, January 6-12,.

Valera, J. E., E. Berger, J. S. Kim, J. E. Reaugh, R. D. Golden, and R. Hofmann, 1978, "Study of Nonlinear Effects on One-Dimensional Earthquake Response," EPRI Report NP-865.

Wong, H. L. and P. C. Jennings, 1975, "Effect of Canyon Topography on Strong Ground Motion," Bu11. Seism. Soc. Amer., Vol. 65, pp. $1239-1258$.

Wong, H. L. and M. D. Trifunac, 1979, "Generation of Artificial Strong Motion Accelerograms," Eartha. Eng. Struc. Dyn., Vor. 7, pp. 509-528. 
There are several approaches available for obtaining complete or approximate responses of layered earth structures. For this problem we use a relatively simple approach to obtain the complete SH-wave response of a plane layered viscoelastic medium. It is well known that for point multipolar sources, the SH-wave part of the motion can be expressed as a cylindrical wave expansion, in which the displacement field in each layer can be written (modified from Ben-Menahem and Singh, 1968) for a cylindrical coordinate system $r, \phi, z$ in the form:

$$
\vec{u}_{n}(\omega)=\sum_{m} \int_{0}^{\infty} u_{n}(m) z_{m} k d k
$$

where

$$
\begin{aligned}
& C_{m}=\operatorname{Real}\left[\left(\vec{e}_{r} \frac{1}{k r} \frac{\partial}{\partial \phi}-\vec{e}_{\phi} \frac{\partial}{\partial k r}\right) \quad J_{m}(k r) e^{i m \phi} \mid\right. \\
& u_{n}^{(m)}(\omega, k)=A_{n}^{(m)} e^{-n_{n} z}+B_{n}^{(m)} e^{n_{n} z}+S_{n}(m) \\
& n_{n}=\left(k^{2}-\rho_{n} \frac{\omega^{2}}{\mu_{n}}\right)^{1 / 2}, \text { Re } n_{n} \geq 0 \\
& A_{n}^{(m)}, B_{n}^{(m)}=\quad \text { the coefficients of downgoing and up- } \\
& \begin{array}{ll}
S_{n}^{(m)}= & \text { particular source contribution in the } \\
& \text { nth layer. }
\end{array} \\
& \rho_{n} \quad=\quad \text { density of nth layer }
\end{aligned}
$$




$\begin{array}{ll}\mu_{n} & =\text { shear modular of } n t h \text { layer } \\ \omega & =\text { Fourier frequency } \\ k & =\text { horizontal wavenumber } \\ \overrightarrow{\mathrm{e}}_{\mathrm{j}} & \quad=\text { unit vector in the } \mathrm{j} \text { direction. }\end{array}$

Defining a traction $\tau_{n}^{(m)}$ by

$$
\tau_{n}^{(m)}(\omega, k)=\mu_{n} \frac{\partial u_{n}^{(m)}}{\partial z},
$$

the boundary conditions at the top and bottom of each layer can be written:

$$
\begin{aligned}
& \tau_{1}^{(m)}(z=0)=0 \\
& \tau_{n-1}^{(m)}\left(z=h_{n-1}\right)=\tau_{n}{ }^{(m)}\left(z=h_{n-1}\right) \\
& u_{n-1}^{(m)}\left(z=h_{n-1}\right)=u_{n}{ }^{(m)}\left(z=h_{n-1}\right)
\end{aligned}
$$

and $B_{N}^{(m)}=0$,

where

$$
\begin{array}{ll}
h_{j} & =\text { depth to the bottom of layer } j \\
B_{N}^{(m)} & =\text { upgoing wave in the underlying halfspace. }
\end{array}
$$

For each azimuthal order $m$ the boundary conditions are sufficient to uniquely determine $A_{n}^{(m)}$ and $B_{n}^{(m)}$. There are two basic numerical approaches for solving for the displacement field $u_{n}^{(m)}$ as a function of depth. We can solve for $u_{n}^{(m)}$ and $\tau_{n}^{(m)}$ explicitly (using Haskell matrices, for example), or, alternatively, we can solve explicitly for the coefficients of upgoing and downgoing waves. Haskell-type matrix methods are desirable for modal problems where all relevant computations can be 
performed with real arithmetic. Since we are interested in solutions for any $0 \geq k \geq \infty$, this benefit is not important; complex arithmetic is a necessity. Here we choose to solve for the coefficients of upgoing and downgoing waves, which can be generated by a very simple recursion relationship.

From here we will drop the superscripts $m$ for brevity and concentrate on the solution for a particular azimuthal order. Let us rewrite the displacement field depth dependence in a slightly altered form

$$
u_{n}(z)=A_{n} e^{-n_{n} \bar{z}}+B_{n} e^{-n_{n}\left(d_{n}-\bar{z}\right)}
$$

where $\bar{z}=z-h_{n-1}$ denotes the local depth relative to the top of the layer, and $d_{n}=h_{n}-h_{n-1}$ is the layer thickness. Note that physically $A_{n}$ is the amplitude of the downgoing wave normalized to the top of layer $n$ and $B_{n}$ is the amplitude of the upgoing wave normalized to the bottom of the layer. This representation contains no growing exponentials $\left(R_{e} n>0\right)$ which, as will be shown, is highly desirable numerically.

Let

$$
p_{n}=e^{-n_{n} d_{n}} \quad \text {. }
$$

Then the boundary conditions given earlier can be recast in the following form:

$$
\left[\begin{array}{cc}
P_{n} & 1 \\
-\mu_{n} n_{n} P_{n} & \mu_{n} n_{n}
\end{array}\right]\left[\begin{array}{l}
A_{n} \\
B_{n}
\end{array}\right]=\left[\begin{array}{cc}
1 & p_{n+1} \\
-\mu_{n+1} n_{n+1} & \mu_{n+1} n_{n+1} P_{n+1}
\end{array}\right]\left[\begin{array}{l}
A_{n+1} \\
B_{n+1}
\end{array}\right]
$$

or 


$$
\left[\begin{array}{c}
A_{n} \\
B_{n}
\end{array}\right]=\frac{1}{P_{n}\left(1+R_{n}\right)}\left[\begin{array}{cc}
1 & R_{n} P_{n+1} \\
R_{n} P_{n} & P_{n} P_{n+1}
\end{array}\right]\left[\begin{array}{l}
A_{n+1} \\
B_{n+1}
\end{array}\right]
$$

where

$$
R_{n}=\frac{\mu_{n} n_{n}-\mu_{n+1} n_{n+1}}{\mu_{n} n_{n}+\mu_{n+1} n_{n+1}}
$$

assuming no source in layer $n$ or $n+1$.

If we define

$$
\left[\begin{array}{l}
A_{n} \\
B_{n}
\end{array}\right]=\left[\begin{array}{c}
\bar{A}_{n} \\
\bar{B}_{n}
\end{array}\right] \quad A_{N} \quad \begin{gathered}
N-1 \\
j=n
\end{gathered} \frac{1}{P_{n}\left(1+R_{n}\right)},
$$

then

$$
\begin{aligned}
& \bar{A}_{n}=\bar{A}_{n+1}+R_{n} P_{n+1} \bar{B}_{n+1} \\
& \bar{B}_{n}=P_{n}\left(R_{n} \bar{A}_{n+1}+\bar{B}_{n+1}\right) \\
& \bar{A}_{N}=1 \\
& \bar{B}_{N}=0
\end{aligned}
$$

Also note that if a layer becomes very thick, then at high wavenumbers [i.e., Real $\left(d_{n} n_{n}\right) \gg 1$, the factor $D_{n}$ underflows and will numerically be set to zero. This causes $\bar{B}_{n} \rightarrow 0$, which is the same as a halfspace radiation condition. This is equivalent to layer-reduction, and it occurs as a natural consequence of the numerical scheme.

Suppose we place a horizontal force at the free surface in the $y$-direction $\left(\phi=90^{\circ}\right) \quad(m=1)$. The recursion above is valid all of the way to the free surface and the free surface condition becomes

$$
-\mu_{1} n_{1} \bar{A}_{1}+\mu_{1} n_{1} \bar{B}_{1} P_{1}=\frac{-F(\omega)}{2 \pi}
$$


or

$$
\left[\bar{A}-P_{1} \bar{B}_{1}\right] A_{N} \prod_{j=1}^{N-1} \frac{1}{P_{j}\left(1+R_{j}\right)}=\frac{F(\omega)}{2 \pi \mu_{1} n_{1}}
$$

Therefore

$$
A_{N}=\frac{F(\omega)}{2 \pi \mu_{1} n_{1}\left[\bar{A}_{1}-P_{1} \bar{B}_{1}\right]} \prod_{j+1}^{N-1} P_{j}\left(1+R_{j}\right)
$$

where $F(\omega)$ is the spectral force applied to the free surface. Note that all $A_{j}, B_{j}$ were previously defined in terms of known functions of $A_{N}$. Therefore

$$
\left[\begin{array}{l}
A_{n} \\
B_{n}
\end{array}\right]=\frac{F(\omega)}{2 \pi \mu_{1} n_{1}\left[\bar{A}_{1}-P_{1} \bar{B}_{1}\right]} \quad \prod_{j=1}^{n-1} P_{j}\left(1+R_{j}\right)\left[\begin{array}{c}
\bar{A}_{n} \\
\bar{B}_{n}
\end{array}\right]
$$

which uniquely determines the entire displacement field. With the complete response due to a surface point force, we can obtain, using reciprocity relationships, the surface response due to any point force or point dislocation source. Details can be found in Fung (1965), or Aki and Richards (1980). Note that the solution requires only one pass through the layer recursion and the solution contains only decaying exponentials.

The above gives a procedure for computing the displacement field for any w,k pair. We must, of course, solve for the two integral transforms to obtain a time series. Here we choose a direct integration of the Fourier-Bessel transform. The obvious solution of this integral involves integration through or near (in the viscoelastic case) poles due to zeroes of the function $\bar{A}_{1}$ $P_{1} \bar{B}_{1}$, the modified Love denominator. We simplify this process by introducing a complex frequency 


$$
\omega=\omega_{R}+i \omega_{I} \quad \omega_{I}<0 \quad .
$$

This increases the stability of the integrand of the Fourier-Bessel transform to the point where equal $k$ spacing with a simple trapezoidal rule may be used with a round-off corrector (Dahlauist and Bjorck, 1974). Using the complex frequency results in a solution which, when transformed with respect to $\omega_{R}$, is the desired solution multiplied by $e^{\omega} I^{t}$ ( $\omega_{I}$ negative). A strong word of caution is necessary at this point. To obtain the final time series, we must multiply by a growing exponential. We must be sure that we are not trying to retrieve the signal out of the noise in the $\omega_{R}$ Fourier transform. Experience has shown that the choice of the artificial imaginary frequency should be closely related to the time window of interest. Experience has also shown that a safe choice seems to be

$$
\left.e^{-\mid \omega} I\right|^{\top}>10^{-2}
$$

where $T$ is the length of the time window.

In other words, using complex frequencies, one should not have to recover more than two orders of magnitude of signal out of the numerical noise. This is certainly dependent on how accurately one evaluates the Fourier-Bessel transform over wavenumber.

It has been noted by Herrmann (1978), that the rigorous expansion of the SH-wave motion in cylindrical harmonics contains some duplications with SV motion. These duplications are nonpropagating contributions which are canceled exactly when the entire motion is computed. When only SH-motion is desired, the derivatives of the Bessel functions, i.e.,

$$
\frac{d J_{m}(k r)}{d k r}=J_{m-1}(k r)-m \frac{J_{m}(k r)}{k r}
$$

should be replaced by 


$$
\frac{d J_{m}(k r)}{d k r} \rightarrow J_{m-1}(k r) .
$$

The substitution above precisely eliminates the SH-wave contributions canceled by the SV-waves in the total solution. Although the modification appears to be a far-field approximation, some nearfield contributions are retained. 
In the nonlinear soil column calculations performed in the present study with the endochronic soil model, the shear modulus and the shear strength were taken to vary with soil depth. In this appendix, the relationships adopted for such depth dependence are developed for dry sand using the data from a cyclic simple shear test to which the endochronic model was fit.

\section{B-1 SHEAR MODULUS}

Generally speaking, the shear modulus of soil depends on both void ratio and confining pressure (Hardin and Drnevich, 1972). An inspection of the cyclic simple shear data for sand, to which the endochronic model was applied in Section 3.2.2., reveals that the slope of the stress-strain curve at the initiation of loading in cycle 1 is essentially the same as the slope of the line through the extremities of the narrow hysterestic loop of cycle 300. This observation implies that the shear modulus of the sand remained essentially fixed at its initial value throughout the shearing process. Although some densification and hardening evidently occurred during the cyclic shear test, leading to an increase in lateral stress and in confining pressure, it must be concluded that the combined effect of void ratio decrease (densification) and increased confining pressure was not of sufficient magnitude to significantly affect the shear modulus. It therefore appears that the change in void ratio during such a cyclic simple shear test is sufficientiy small that its affect on the shear modulus can be disregarded. A similar conclusion for sand in such a device has been noted also by others (Bazant, Krizek and Shieh, 1979). Therefore, if it is assumed that the void ratio does not significantly decrease with depth, the variation of the shear modulus with depth will result solely from its dependence on confining pressure. 
Extensive experimental data covering a reasonably wide range of confining pressures have shown that the shear modulus of sand is essentially proportional to the square root of the confining pressure (Duffy and Mindlin, 1957; Harden and Richart, 1963; and Drnevich, Hall and Richart, 1967). This relationship is demonstrated, for example, in Figure B.1 which shows the effect of confining presure on the shear modulus of Ottawa sand over a pressure range from 300 psf (2.1 psi) to 10,000 psf (69 psi). The dependence of the shear modulus $G$ of sand on the confining pressure $\sigma$ can therefore be expressed in the form:

$$
G=G_{R} \sqrt{\frac{\sigma}{\sigma_{R}}}
$$

where $G_{R}$ denotes the shear modulus at some reference confining pressure $\sigma_{R}$.

In a vertical column of cohesionless sand, the confining pressure results primarily from the gravitational effect, with some small contribution from the atmospheric pressure, which will be neglected here as in previous work by others (Idriss and Seed, 1968; Streeter, Wylie and Richart, 1974). Since the vertical stress, $\sigma_{v}$, at depth $d$ in a soil deposit of uniform density is given by the expression:

$$
\sigma_{V}=g \rho d
$$

where $g$ denotes the gravitational acceleration and $\rho$ is the mass density of the soil, the confining pressure at $d$ can be expressed as:

$$
\sigma=\left(g_{\rho} d\right) \frac{\left(1+2 K_{0}\right)}{3}
$$

where $K_{0}$ is the coefficient of earth pressure. Typical values of $K_{0}$ for normally consolidated sands are in the range 0.3 to 0.5 . Adopting the median value of $K_{0}=0.4$, Equation $B-3$ becomes 


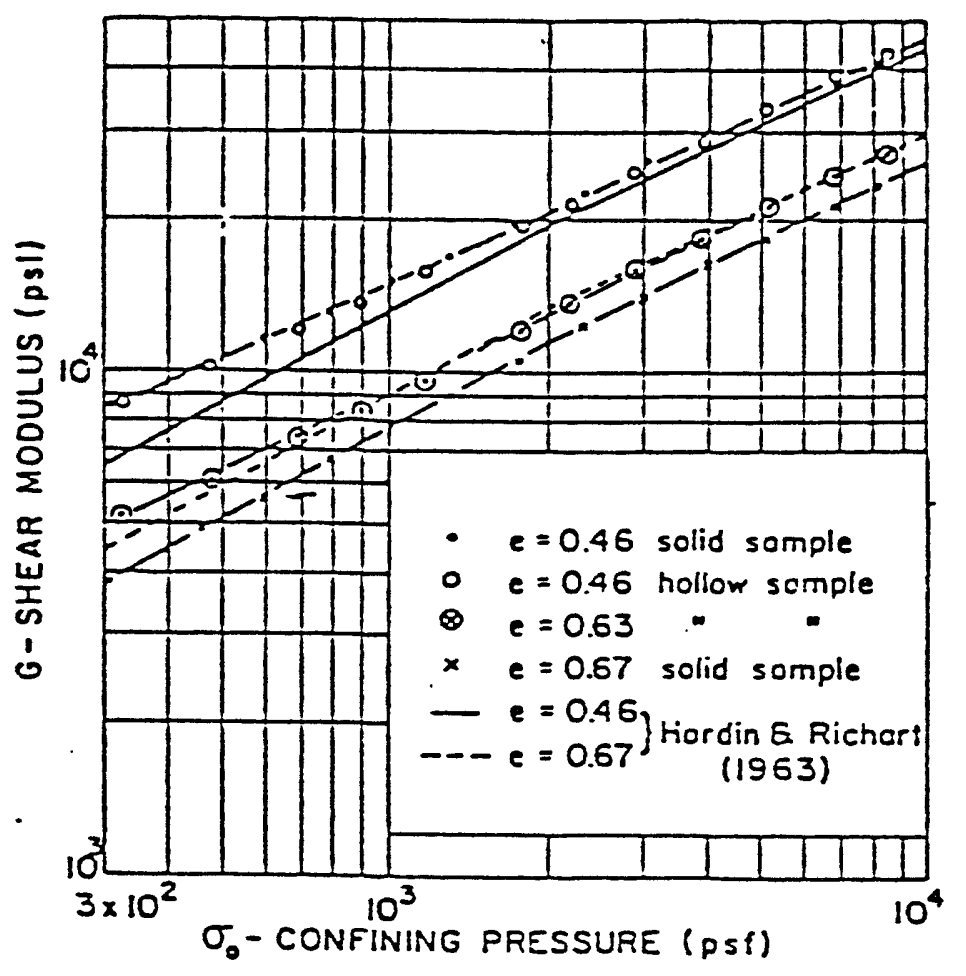

Figure B.1. Effect of confining pressure on the shear modulus of Ottawa Sand (from Drnevich, Hall and Richart, 1967). 


$$
\sigma=0.6 \mathrm{god}
$$

which then combined with Equation B-1 gives the result:

$$
G=\alpha \sqrt{d}
$$

where we have set:

$$
\alpha=G_{R} \sqrt{\frac{0.6 \rho g}{\sigma_{R}}}
$$

We now evaluate $\alpha$ from the cyclic simple shear data to which the endochronic model was fit. Let us note first of all that the data were obtained from a Norwegian Geotechnical Institute type of simple shear device, which employs a cylindrical soil sample enclosed in a wire-reinforced membrane fitted with a base and a cap. The base is attached to a stiff plate which is subjected to cyclical motions perpendicular to the sample axis, while the cap is held stationary. Also, a constant uniform compressive vertical stress, $\sigma_{v}$, is applied to the sample throughout the test in the direction of the sample axis. A sample undergoing shear in such a device can be considered to deform with essentially no lateral strain, since the wire reinforcement prevents such deformation. The lateral stress, $\sigma$, developed by the interaction between the sand and the wire-reinforced membrane can therefore be related to the applied vertical stress, $\sigma_{v}$, by the coefficient of earth pressure, $k_{0}$, i.e.,

$$
\sigma_{\ell}=K_{0} \sigma_{v}
$$

The confining pressure in the simple shear test, which we shall take here as the reference confining pressure, $\sigma_{R}$, can then be expressed as:

$$
\sigma_{R}=\frac{\sigma_{v}\left(1+2 K_{0}\right)}{3}
$$


which, for $k_{0}=0.4$, gives the result:

$$
\sigma_{R}=0.6 \sigma_{v} \quad \text {. }
$$

For the cyclic simple shear test of interest, we have:

$$
\begin{aligned}
& \sigma_{V}=191.52 \mathrm{kPa} \quad \rho=1462 \mathrm{~kg} / \mathrm{m}^{3} \\
& G_{R}=19.75 \mathrm{MPa} .
\end{aligned}
$$

Upon substituting these values into Equation $B-6$, we find

$$
\alpha=0.54 \mathrm{MPa} \mathrm{cm}-1 / 2
$$

Therefore, Equation B-5, with a defined above, describes the dependence of the shear modulus on depth employed in the nonlinear soil column calculations discussed herein. This dependence is depicted graphically in Figure B-2. As pointed out in Section 4.2, the value of $G_{R}$ used here is actually a factor of 2 too small to fit the cyclic shear test, reflecting an error in Valanis and Read (1980) which was not discovered until after the numerical calculations were completed.

\section{B-2 SHEAR STRENGTH}

Extensive experimental results show that the shear strength of normally consolidated sands, unless exceptionally loose, is essentially proportional to the normal stress acting on the plane (or planes) of failure. Such a relationship follows also from the Mohr-Coulomb failure theory, and for the case of simple shear it can be expressed in the form:

$$
s_{f}=\sigma_{n} \tan \phi
$$


where $s_{f}$ represents the shear stress at failure, $\sigma_{n}$ is the normal stress acting on the plane(s) of failure, and $\phi$ is the Mohr-Coulomb friction angle.

The dependence of shear strength on normal stress described above can be easily incorporated into the endochronic model of simple shear described in Section 3.2. Toward this end, let us note, first of $\mathbf{1} 11$, that for monotonic loading, Equation B-6 reduces to the following form:

$$
s=H \operatorname{Erf}(\sqrt{k z})
$$

where $z$ is the intrinsic time scale, Erf ( ) denotes the error function, and the hardening function $H$ is given by the expression:

$$
H=H_{0}+\left(H_{\infty}-H_{0}\right)\left(1-e^{-n Z}\right)
$$

Here, $H_{0}, H_{\infty}$ and $n$ are constants, whose values are listed in Section 3.2.2. To obtain the shear stress at failure for this model, one simply takes the limit of the right hand side of Equation $B-13$ as $z$ goes to infinity. This leads to the result:

$$
s_{f}= \begin{cases}H_{0}, & \text { if } n=0 \\ H_{\infty}, & \text { if } n \neq 0\end{cases}
$$

Therefore, in order to develop a general representation that encompasses all values of $n$, we take both $H_{0}$ and $H_{\infty}$ to be proportional to $\sigma_{n}$, in order to satisfy Equations $B-12$ and $B-15$. This leads to the following expression for $\mathrm{H}$ :

$$
H=\frac{\sigma_{n}}{\sigma_{n}^{0}}\left[H_{0}+\left(H_{\infty}-H_{0}\right)\left(1-e^{-n Z}\right)\right]
$$

where $H_{0}$ and $H_{\infty}$ are the hardening coefficients corresponding to the reference normal stress, $\sigma_{n}^{0}$. 
In the simple shear test, from which the data depicted in Figure 3-1 were obtained, the normal stress acting on the failure planes was equal to the applied vertical stress, since the planes of failure in such a specimen are perpendicular to its axis. In the particular simple shear test of interest here, the applied vertical stress was $191.5 \mathrm{kPa}(4,000 \mathrm{psf})$, so that the reference normal stress, to which the values of $H_{0}$ and $H_{\infty}$ given in Section 3.2.2. correspond, is:

$$
\sigma_{n}^{0}=191.5 \mathrm{kPa} .
$$

In a soil column subjected to vertically traveling SH waves, the failure planes of the soil are perpendicular to the gravitational field. The normal stress on such planes, for a soil element at depth $d$ from the free surface, is then given by the expression:

$$
\sigma_{n}=g o d
$$

where both $g$ and $o$ have been previously defined. Using this result, Equation B-16 can be written in the form:

$$
\cdot H=\beta d\left[H_{0}+\left(H_{\infty}-H_{0}\right)\left(1-e^{-n z}\right)\right]
$$

where we have set:

$$
\beta=\left(\frac{g_{\rho}}{\sigma_{n}^{0}}\right)
$$

For the soil data of interest, $\rho=1462 \mathrm{~km} / \mathrm{m}^{3}$ and $\sigma_{n}^{0}=191.5$ $\mathrm{kPa}$, which leads to the following value for $\mathrm{B}$ :

$$
B=7.49 \times 10^{-4} \mathrm{~cm}^{-1} \text {. }
$$

Therefore, the proportionality between shear strength and normal stress, specified in Equation B-12, is incorporated into the 
endochronic model of simple shear by taking the hardening function in the form of Equation B-19, with $B$ defined by Equation B-21 and $H_{0}, H_{\infty}$ and $n$ assigned the values listed earlier in Section 3.2.2. For the soil column calculations performed in this study, the above result leads to the linear dependence of shear strength on depth shown in Figure B.2. Parenthetically, it is noted that the above results are in agreement with previous studies of soil amplification based on truly non-linear soil models in which the shear strength was taken to increase with depth. (Joyner and Chen, 1975; Streeter, Wylie and Richart, 1974).

\section{B-3 EQUIVALENT LINEAR SHEAR MODULUS}

The SHAKE code used in part of the numerical simulations performed in the present study utilizes an equivalent linear shear model to describe nonlinear soil behavior. The model is completely defined in terms of an equivalent shear modulus, $G_{\text {eq, }}$ and a damping factor, $B$; the shear strength does not enter the mode 1 explicitly. $G_{e q}$ is defined as the secant modulus for monotonic shear loading, while $\beta$ is defined in terms of the area enclosed within a complete, symmetric hysteresis cycle. Both $G_{\text {eq }}$ and $B$ will in general depend on the shear strain, $\gamma$. Furthermore, $G_{e q}$ and the elastic shear modulus $G$ discussed in Section B-1 are equal oniy at $\gamma=0$.

The SHAKE code allows for the dependence of soil properties on depth by taking $G_{\text {eq }}$ to be proportional to the square root of the confining pressure, $\sigma$. The specific form of this dependence adopted in SHAKE is:

$$
G_{e q}=K_{S}(\gamma) \sqrt{\sigma}
$$

where $K_{S}(\gamma)$ provides for the dependence of $G_{e q}$ on $\gamma$. 

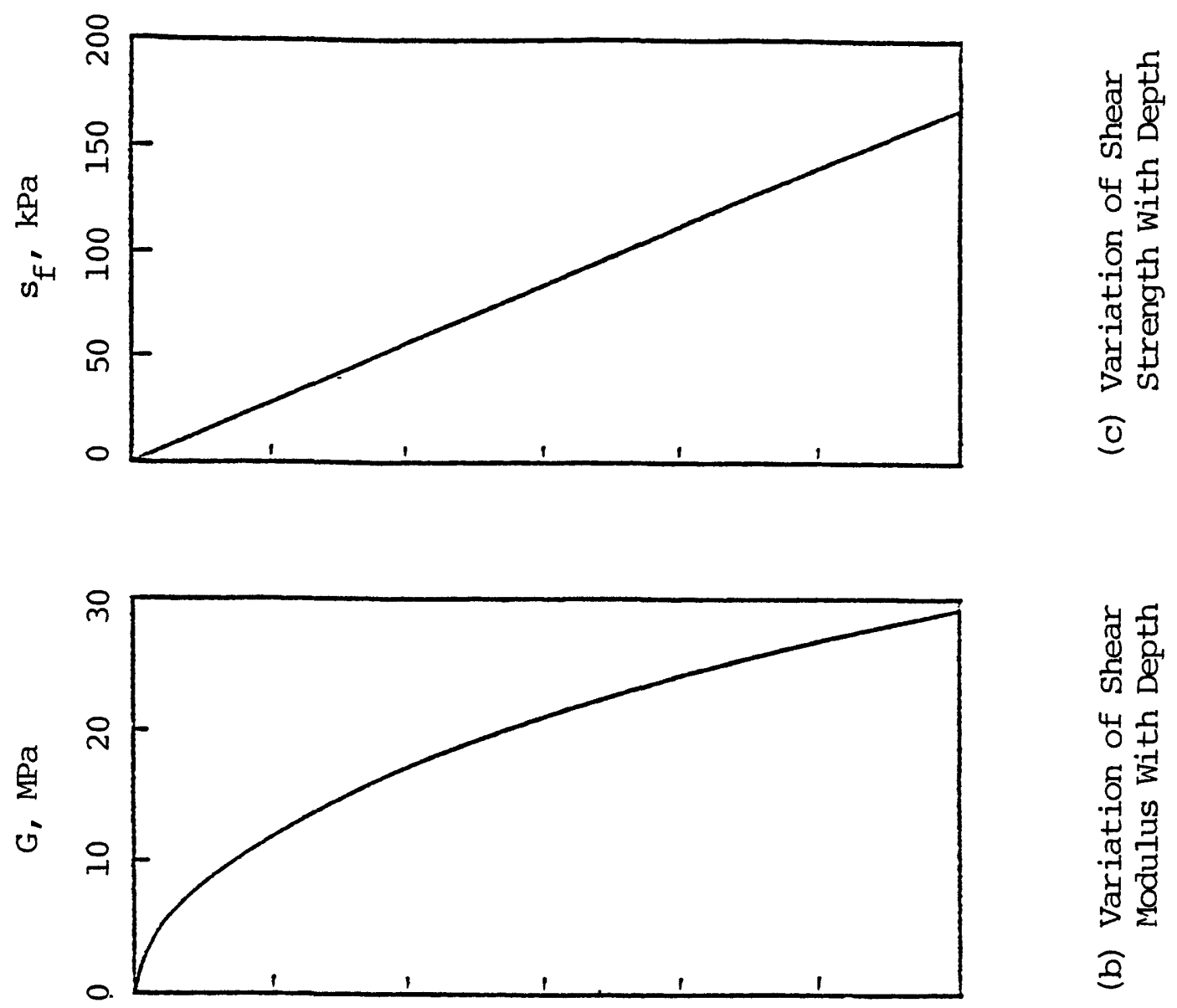

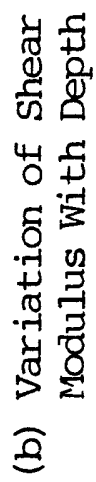

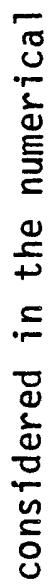

产

동 훙

造

4

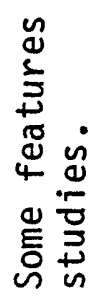

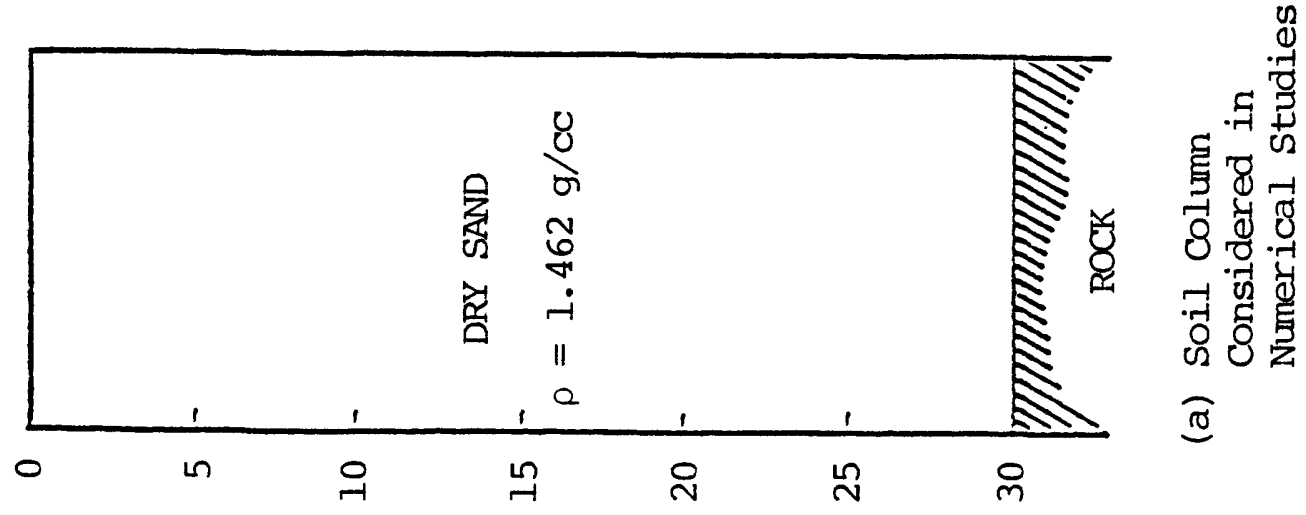

$\stackrel{0}{0}$
0
$\frac{0}{5}$
$\frac{\sigma}{4}$

(ur) H山dتם 
For shear behavior characteristic of soils, the equivalent shear modulus, $G_{e q}$, can be related, at least in principle, in a nonlinear manner to the shear strength, $s_{f}$, the elastic shear modulus, $G$, and the strain, $r$, i.e.,

$$
G_{e q}=G_{e q}\left(s_{f}, G, r\right) \text {. }
$$

In the preceding sections of this appendix, concepts from soil mechanics were used to develop expressions for the dependences of $s_{f}$ and $G$ on the confining pressure $\sigma$, which were then incorporated into the nonlinear endochronic model. These expressions can be placed in the following forms:

$$
\begin{aligned}
& G=\alpha * \sqrt{\sigma} \\
& S_{f}=\beta * \sigma
\end{aligned}
$$

where $\alpha^{*}$ and $\beta^{*}$ are constants, not to be confused with $\alpha$ and $\beta$ introduced earlier. On the basis of the above discussion, the following question may be raised: How compatible is the dependence of $G_{e q}$ on $\sigma$, assumed in the SHAKE code and expressed by Equation B-22, with Equation B-23, when Equations B-24 are utilized.

To answer this question, it proves convenient to employ a constitutive model which is analytically simpler to deal with than the endochronic model but also provides equivalent modeling capability, at least for monotonic shear loading processes. A model which meets these requirements is the well-known "hyperbolic" soil model (Konder, 1963), defined by the equation:

$$
s / s_{f}=\frac{\gamma / \gamma_{R}}{1{ }^{+}{ }_{\gamma} / \gamma_{R}}
$$

where $\gamma_{R}$, the reference strain, is defined as:

$$
\gamma_{R}=s_{f} / G \quad
$$


Since the equivalent shear modulus, $G_{e q}$, is defined as the secant modulus, we can write:

$$
G_{e q}=s / r \quad \text {. }
$$

Upon introducing this result into Equation $\mathrm{B}-25$, and rearranging, we obtain the expression:

$$
G_{e q}=\frac{G}{1+\gamma / \gamma_{R}}
$$

If we now substitute Equations $B-24$ into Equations $B-25$ and $B-28$, the following expression results for the general dependence of $G_{\text {eq }}$ on $\sigma$ :

$$
G_{e q}=\alpha^{\star} \sqrt{\sigma}\left(1+\frac{\gamma}{\delta^{\star} \sigma}\right)^{-1}
$$

where we have set $\delta^{*}=\beta^{\star} / \alpha^{*}$.

Consider now the case of small strains. In this instance, the second term inside the bracket of Equation B-29 can be neglected, so long as $\sigma$ is sufficiently larger than zero, leading to the expression:

$$
G_{e q}=\alpha \sqrt{\sigma}
$$

which agrees with the form of the dependence of $G_{e q}$ on $\sigma$ adopted in SHAKE. For large strains, however, Equation B-29 reduces to the form:

$$
G=\frac{8 *_{\sigma}}{r}
$$


which exhibits a different dependence of $G_{e q}$ on $\sigma$ than employed in SHAKE. It therefore appears that the relatively simple expression adopted in the SHAKE code to describe the dependence of $G_{\text {eq }}$ on $\sigma$ is in agreement with the nonlinear model only at low strains and progressively deviates as the strain increases. 


\section{APPENDIX C \\ COMPUTER SUBROUTINE FOR SIMPLE SHEAR}

A FORTRAN subroutine called ENDO was developed to solve the constitutive equations governing simple shear, described in Section 3.2.1., using the numerical method outined in Section 4.3.1. The program, which is listed on the following pages, runs on the $s^{3}$ UNIVAC 1100/80, and was incorporated into the SWIS finite element code. 


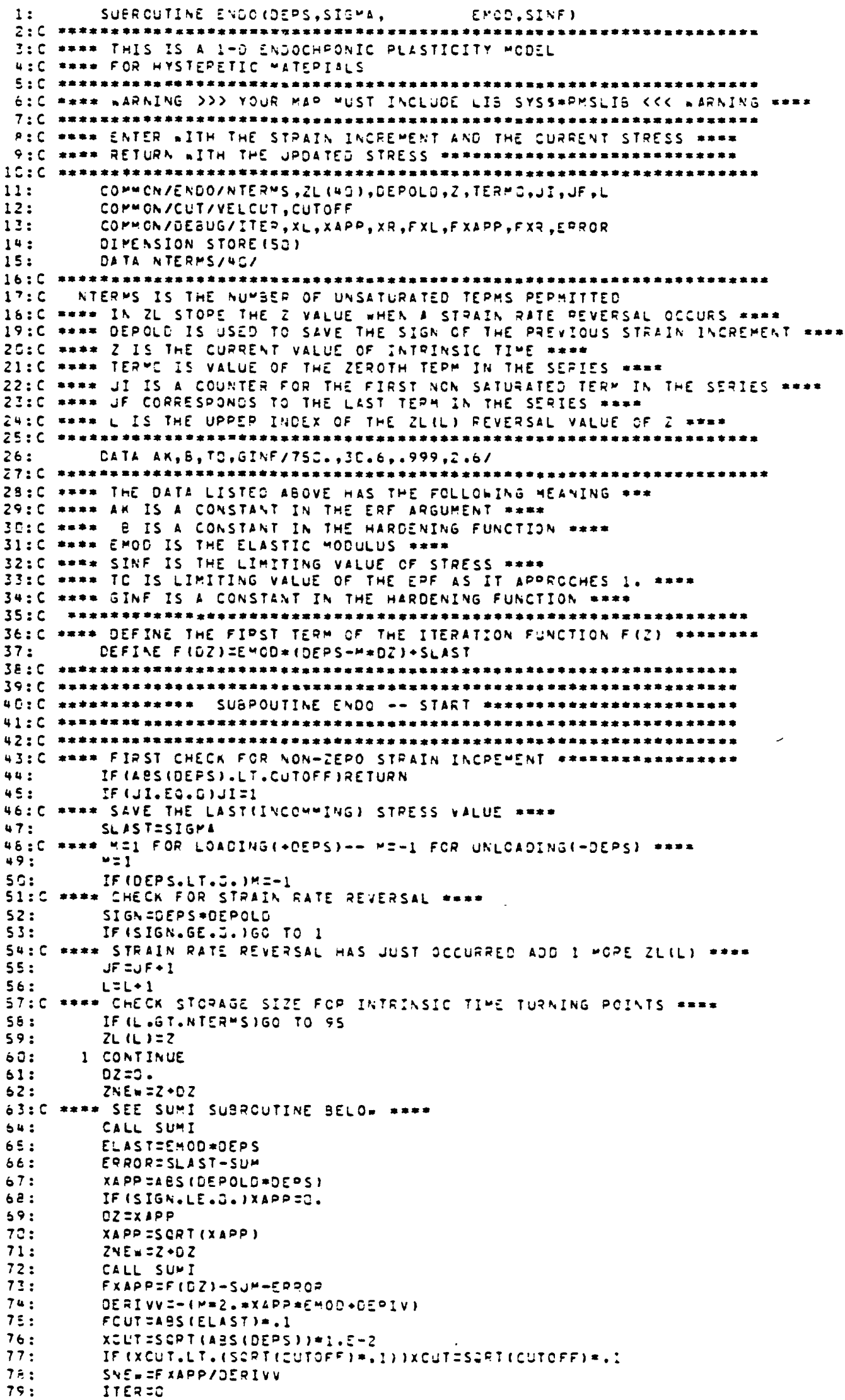




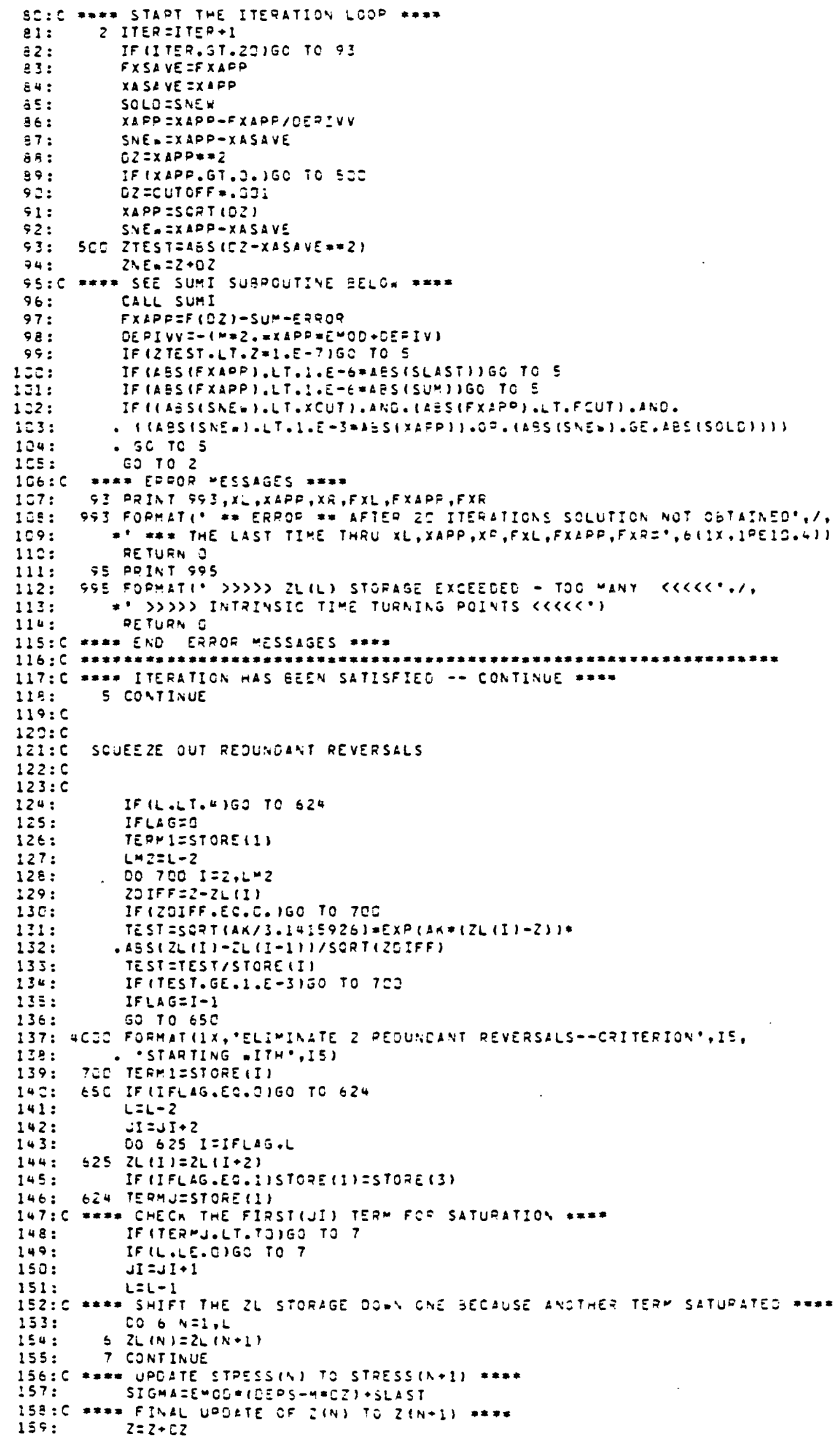




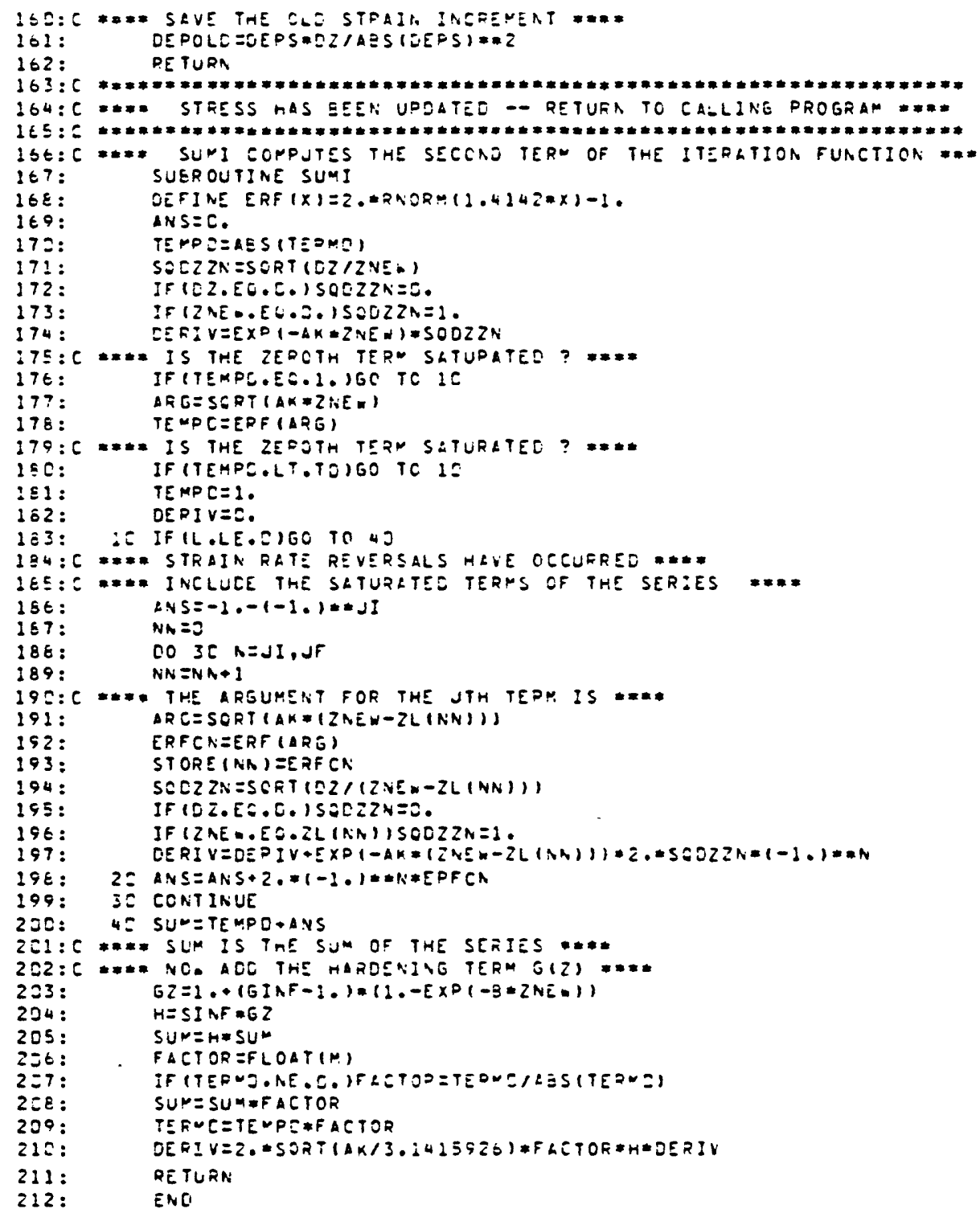




\section{APPENDIX D}

THE NEW ENDOCHRONIC SOIL MODEL:

AN ASSESSMENT OF ITS PRACTICAL UTILITY FOR

USE IN TWO- AND THREE-DIMENSIONAL GROUND

MOTION CODES

\section{D-1. INTRODUCTION}

A new endochronic theory of plasticity was recently developed (Valanis, 1979) which is free of several undesirable features inherent in earlier versions of the theory, and provides increasing modeling capability with substantially fewer material parameters. This new constitutive theory is fully three-dimensional, and has the capability to describe the important features of soil response to seismic loading, such as hysteresis, densification/dilatancy, strain hardening/softening, and cyclic creep (ratcheting). It predicts instantaneous elastic response at points where loading, unloading or reloading begin and, for one-dimensional unload-reload processes, produces hysteresis loops that close, no matter how small the magnitude of the unloading.

Initially, the new endochronic theory was applied to several relatively simple deformations of soils, such as hydrostatic compression and cyclic simple shear (Valanis and Read, 1979; 1980), and for this purpose appropriate numerical schemes were developed to treat the governing one-dimensional equations in a modern, high speed computer. In the present investigation, further work was done to make the numerical scheme for simple shear response suitable for use in conjunction with a nonlinear wave propagation code (SWIS), so that the endochronic model could deal with the complex strain histories produced in the soil by the earthquake signature without requiring unreasonably large computing time and computer storage. 
Recently, the feasibility of using the two- and threedimensional versions of the new endochronic model in conjunction with large finite element codes to analyze multidimensional ground motion problems has been considered. The purpose of this appendix is to point out those features of the new endochronic theory which pose serious computational difficulties, and to briefly present a very recently proposed and promising computational approach which appears to circumvent these difficulties.

\section{D-2. NOTATION AND DEFINITIONS}

For isotropic materials undergoing small deformations, the stress $\sigma_{i j}$ may be decomposed into a deviatoric component, $s_{i j}$, and a hydrostatic component, $\sigma$, in the following manner:

$$
\sigma_{i j}=s_{i j}+\sigma \delta_{i j}
$$

where $\sigma=1 / 3 \sigma_{i j}$ and $\delta_{i j}$ is the kronecker delta. Similarly, the incremental small strain tensor, $d \varepsilon_{i j}$, may be decomposed into a deviatoric component, $d_{i j}$, and a volumetric component, $d_{\varepsilon}$ :

$$
d \varepsilon_{i j}=d e_{i j}+1 / 3 d \varepsilon \delta_{i j}
$$

where $d_{\varepsilon}=d \varepsilon_{i j}$. Upon using an incremental form of Hooke's law, the increment of the plastic strain, $d \varepsilon_{j}{ }_{j}$, may be defined as follows:

$$
d \varepsilon_{i j}^{P}=d \varepsilon_{i j}-C_{i j k} d \sigma_{k_{\ell}}
$$

where $C_{i j k_{\ell}}$ is given by the expression:

$$
C_{i j k_{\ell}}=\left(\frac{1}{9 K_{0}}-\frac{1}{6 \mu_{0}}\right) \delta_{i j} \delta_{k_{l}}+\frac{1}{4_{\mu_{0}}}\left(\delta_{i k} \delta_{j \ell}+\delta_{j k} \delta_{i \ell}\right)(D-4)
$$

In the above equation, $\mu_{0}$ and $K_{0}$ denote the elastic shear and bulk moduli, respectively. 
The plastic strain increment, $d \varepsilon_{j}^{p}$, may also be decomposed into a deviatoric component, $d \theta_{i j}$, and a volumetric component, $d \theta$, i.e.,

$$
d \varepsilon_{i j}^{P}=d \theta_{i j}+1 / 3 d \theta \delta_{i j}
$$

where $d \Theta=d \varepsilon_{i j}^{P}$. From Equations $0-3$ to $0-5$, it then follows that:

$$
\begin{aligned}
d \sigma_{i j} & =\left\lceil K_{0}(d \varepsilon-d \theta)-2 / 3 \mu_{0} d \varepsilon\right\rceil \delta_{i j} \\
& +2 \mu_{0}\left(d \varepsilon_{i j}-d \theta_{i j}\right)
\end{aligned}
$$

\section{D-3. BASIC EQUATIONS OF NEW ENDOCHRONIC THEORY}

The new endochronic theory is based on the hypothesis that the current state of stress is a linear functional of the entire history of plastic strain, with the history defined with respect to a time scale (intrinsic time) which is itself a property of the material at hand. The basic equations of this new theory, which describe the general, three-dimensional behavior of isotropic, rate-independent materials undergoing small, isothermal deformations, are summarized below, using the notation defined above:

$$
\begin{aligned}
\sigma_{i j} & =H_{D} \int_{0}^{z_{0}} \rho\left(z_{D}-z^{\prime}\right) \frac{\partial \theta_{i j}}{\partial z^{\prime}} d z^{\prime} \\
& +\delta_{i j} H_{H} \int_{0}^{z_{H}} \phi\left(z_{H}-z^{\prime}\right) \frac{\partial \theta}{\partial z^{\prime}} d z^{\prime}
\end{aligned}
$$

and 


$$
\begin{aligned}
d \sigma_{i j} & =\left[K_{0}(d \varepsilon-d \theta)-2 / 3 \mu_{0} d \varepsilon\right] \delta_{i j} \\
& +2 \mu_{0}\left(d \varepsilon_{i j}-d \theta_{i j}\right)
\end{aligned}
$$

where the intrinsic time scales, $z_{D}$ and $z_{H}$, are positive, monotonically increasing quantities defined by the following expressions:

$$
\begin{aligned}
& d z_{D}^{2}=k_{00} d \zeta_{D}^{2}+k_{01} d \zeta_{H}^{2} \\
& d z_{H}^{2}=k_{10} d \zeta_{D}^{2}+k_{11} d \zeta_{H}^{2}
\end{aligned}
$$

and $H_{D}, H_{H}$ are appropriate hardening/softening functions. In the above equations, the intrinsic time measures, $d \zeta_{D}$ and $d \zeta_{H}$, are defined as follows:

$$
\begin{aligned}
& d \zeta_{D}^{2}=d \theta_{i j} d \theta_{i j} \\
& d \zeta_{H}^{2}=|d \theta|^{2}
\end{aligned}
$$

and the $k_{r s}$ are elements of the material-dependent coupling matrix $[k]$, which may depend on $\zeta_{D}$ and $\zeta_{H} ;$ also, the vertical bars enclosing a symbol denote its absolute value. It is evident that since $d z_{D}$ and $d z_{H}$ are independent of the natural time scale given by a clock, the materials described by the above equations are plastic strain history dependent but strain rate independent.

Equations D-7 to D-12 form a system of coupled nonlinear equations which completely describe the new endochronic theory once the kernel functions, $o(z)$ and $\phi(z)$, and the hardening/softening functions, $H_{D}$ and $H_{H}$, have been specified for a given material. The kernel functions consist of infinite series of exponentials, i.e., 


$$
\begin{aligned}
& \rho(z)=\sum_{r=1}^{\infty} R_{r} e^{-\beta} r^{z} \\
& \phi(z)=\sum_{r=1}^{\infty} P_{r} e^{-\lambda r}
\end{aligned}
$$

subject to the conditions $\rho(0)=\phi(0)=\infty$.

It may prove beneficial for numerical purposes to have an incremental form of Equation $0-7$, which can be obtained by differentiation to give the following expression:

$$
\begin{aligned}
d \sigma_{i j} & =\left[H_{D} \int_{z}^{z_{D}} \rho\left(z_{D^{-}} z^{\prime}\right) \frac{\partial^{2} \theta_{i j}}{\partial z^{\prime 2}}\right] d z_{D} \\
& +\left[\delta_{i j} H_{H} \int_{0}^{z_{H}} \phi\left(z_{H}-z^{\prime}\right) \frac{\partial^{2} \theta}{\partial z^{\prime 2}}\right] d z_{H}
\end{aligned}
$$

where we have assumed that $H_{D}$ and $H_{H}$ are relatively insensitive to small changes in $z_{D}$ and $z_{H}$.

\section{D-4. SOURCE OF COMPUTATIONAL DIFFICULTIES}

For the purpose of delineating the computational difficulties posed by the above system of equations, it is sufficient, and more convenient, to restrict attention to the deviatoric part of the model, since this is the major source of difficulty in treating the model computationally. For the special case of deviatoric response, the general equations given above for the new endochronic model reduce to the following set of equations:

$$
s_{i j}=\int_{0}^{z} \rho\left(z-z^{\prime}\right) \frac{\partial \theta_{i j}}{\partial z^{\prime}} d z^{\prime}
$$




$$
\begin{aligned}
& d s_{i j}=2 G\left(d e_{i j}-d \theta_{i j}\right) \\
& d z^{2}=d \theta_{i j} d \theta_{i j}
\end{aligned}
$$

subject to the constraints that $\operatorname{tr}\left(s_{i j}\right)=\operatorname{tr}\left(d s_{i j}\right)=0$.

Let us assume that the deviatoric strain histories, $e_{i j}(t)$, are given, and we wish to solve the above equations, in an incremental numerical manner, for the corresponding deviatoric stress histories, $s_{i j}(t)$. Considering, first of all the case of one-dimensional deformation (simple shear), it can be shown that the quantity $d \theta / d z$ will have either one of two values, namely, +1 when de $>0$ or -1 when de<0. Consequently, the evaluation of the integral in Equation (D-16) for this case can be performed analytically for suitable (integrable) forms of the kernel function $o\left(z-z^{\prime}\right)$, and we have demonstrated this in recent work (Valanis and Read, 1979; 1980).

Turning now to the case of two- and three-dimensional deformations, the situation suddenly becomes much more complicated, since the quantities $d \theta_{i j} / d z$ now represent the direction cosines between the vector $d z$ and the vector $d \theta_{i j}$ in a multidimensional plastic strain space. These direction cosines vary in some (a priori unknown) manner with $z$, which depends on the deformation path. Consequently, since the dependence of $d \theta_{i j} / d z$ on $z$ is not known in advance, the evaluation of the integrals given in Equation (D-16) cannot be performed analytically for two- and three-dimensional response, as was possible for the one-dimensional case. Moreover, it now becomes necessary to store in computer storage the histories of the terms $d \theta_{i j} / d z$ with respect to $z$, in order to be able to numerically evaluate the integrals in Equation (D-16).

On the basis of the above observations, the following conclusions are drawn regarding the feasibility of using the system of endochronic equations given by (D-7) to (D-12) in conjunction with large finite element codes to analyze two- and three-dimensional problems in ground motion: 


\section{Computer Storage Requirements}

Because of the hereditary nature of the integrals in the new endochronic theory, the histories, or some significant portions thereof, of the terms $d \theta_{i j} / d z$ must be stored for each computational zone in the computer memory as they evolve during the course of a calculation. If these integrals exhibit "fading memory," as they will if the kernel function $\rho(z)$ approaches an asymptote as $z$ becomes increasingly large, then it is necessary only to retain the histories of the $d \theta_{i j} / d z$ beyond some non-zero value of $z$. Nonetheless, even the requirement to retain significant portions of the histories of the $d \theta_{i j} / d z$ for each computational zone in a problem poses presently intractible computer storage requirements for most problems of practical interest.

\section{Computational Speed}

Inasmuch as the terms $d \theta_{i j} / d z$ are not known as functions of $z$ at the outset, but are determined as part of the solution, the hereditary integrals, which in the one-dimensional case could be evaluated analytically (Valanis and Read, 1979; 1980), must be evaluated numerically for twoand three-dimensional response; this proves to be very costly and computationaliy inefficient. The situation becomes even more serious if iteration techniques are employed to solve the system of equations, since in the process of iterating toward an acceptable solution, the algorithm may require that the integrals be evaluated several times for each incremental strain step imposed; this process is repeated, then, for every computation zone in a problem which results in a prohibitively slow computing speed when compared with other advanced inelastic soil models. 


\section{D-5. RECENT DEVELOPMENTS}

Very recently, a numerical approach which avoids the above computational difficulties was proposed by the senior author, and an equivalent approach was independently proposed by Valanis (1980). This approach uses approximate forms for the singular kernel functions, at the cost of some accuracy, which appears to be minimal. Inasmuch as this approach is presently under investigation at $\mathrm{s}^{3}$, we shall provide only a brief outline of the method below, and for this purpose only the deviatoric part of the endochronic model will be considered.

In essence, the approach consists of approximating the kernel function $\rho(z)$, defined in Equation $D-13$, by a finite series of exponentials and by satisfying the singular condition $\rho(0)=$ approximately by taking $\rho(0)$ to be some large finite number. Depending on the range of $z$ and the accuracy required, the number of terms required in the series may indeed be very small, as recent preliminary studies have shown. Using this approximation for $o(z)$, it can be shown (Valanis, 1980) that the governing endochronic equations for deviatoric response (see Equations (D-16) to (D-18)) can be rediced to the following system of differential equations:

$$
\begin{aligned}
& d s_{i j}=\sum_{r=1}^{n} d 0_{i j}^{r} \\
& d Q_{i j}^{r}=R_{r} d \theta_{i j}-\beta_{r} 0_{i j}^{r} d z \\
& d s_{i j}=2 G\left(d e_{i j}-d \theta_{i j}\right) \\
& d z^{2}=d \theta_{i j} d \theta_{i j}
\end{aligned}
$$

where $R_{r}$ and $\beta_{r}$ have been defined previously (see Equation $0-13$ ). 
Upon setting

$$
\begin{aligned}
\bar{R} & =\frac{1}{2 G} \sum_{r=1}^{n} R_{r} \\
\bar{Q}_{i j} & =\frac{1}{2 G} \sum_{r-1}^{n} B_{r} Q_{i j}^{r}
\end{aligned}
$$

and combining Equations (D-19) to (D-21), one can show that

$$
d e_{i j}+\bar{Q}_{i j} d z=(1+\bar{R}) d \theta_{i j}
$$

By taking the norm of both sides, and rearranging, we obtain the result:

$$
\left[\|\bar{Q}\|^{2}-(1+\bar{R})\right] d z^{2}+2 d e_{i j} \overline{0}_{i j} d z+\|d e\| \|^{2}=0
$$

where the double bars enclosing a symbol denote its norm. Equation (D-26) is a quadratic equation for $d z$, from which $d z$ can be easily determined if $\mathrm{de}_{i j}$ is specified. The development of a numerical scheme from this approach is straightforward. Note that the hereditary integrals have been done away with and the computer storage requirements are very small. 